\title{
MODULARITY OF GENERATING SERIES OF DIVISORS ON UNITARY SHIMURA VARIETIES II: ARITHMETIC APPLICATIONS
}

\author{
JAN H. BRUINIER, BENJAMIN HOWARD, STEPHEN S. KUDLA, \\ MICHAEL RAPOPORT, AND TONGHAI YANG
}

\begin{abstract}
We prove two formulas in the style of the Gross-Zagier theorem, relating derivatives of $L$-functions to arithmetic intersection pairings on a unitary Shimura variety. We also prove a special case of Colmez's conjecture on the Faltings heights of abelian varieties with complex multiplication. These results are derived from the authors' earlier results on the modularity of generating series of divisors on unitary Shimura varieties.
\end{abstract}

\section{Contents}

1. Introduction

2. Small CM cycles and derivatives of $L$-functions

3. Further results on the convolution $L$-function

4. Big CM cycles and derivatives of $L$-functions

5. Faltings heights of $\mathrm{CM}$ abelian varieties

References

\section{INTRODUCTION}

Fix an integer $n \geqslant 3$, and a quadratic imaginary field $\boldsymbol{k} \subset \mathbb{C}$ of odd discriminant $\operatorname{disc}(\boldsymbol{k})=-D$. Let $\chi_{\boldsymbol{k}}: \mathbb{A}^{\times} \rightarrow\{ \pm 1\}$ be the associated quadratic character, let $\mathfrak{d}_{\boldsymbol{k}} \subset \mathcal{O}_{\boldsymbol{k}}$ denote the different of $\boldsymbol{k}$, let $h_{\boldsymbol{k}}$ be the class number of $\boldsymbol{k}$, and let $w_{\boldsymbol{k}}$ be the number of roots of unity in $\boldsymbol{k}$.

By a hermitian $\mathcal{O}_{\boldsymbol{k}}$-lattice we mean a projective $\mathcal{O}_{\boldsymbol{k}}$-module of finite rank endowed with a nondegenerate hermitian form.

1991 Mathematics Subject Classification. 14G35, 14G40, 11F55, 11F27, $11 \mathrm{G} 18$.

Key words and phrases. Shimura varieties, Borcherds products, arithmetic intersection theory.

J.B. was supported in part by DFG grant BR-2163/4-2. B.H. was supported in part by NSF grants DMS-1501583 and DMS-1801905. M.R. was supported in part by the Deutsche Forschungsgemeinschaft through the grant SFB/TR 45. S.K. was supported by an NSERC Discovery Grant. T.Y. was supported in part by NSF grant DMS-1500743. 
1.1. Arithmetic theta lifts. Suppose we are given a pair $\left(\mathfrak{a}_{0}, \mathfrak{a}\right)$ in which

- $\mathfrak{a}_{0}$ is a self-dual hermitian $\mathcal{O}_{\boldsymbol{k}}$-lattice of signature $(1,0)$,

- $\mathfrak{a}$ is a self-dual hermitian $\mathcal{O}_{\boldsymbol{k}}$-lattice of signature $(n-1,1)$.

This pair determines hermitian $\boldsymbol{k}$-spaces $W_{0}=\mathfrak{a}_{0 \mathbb{Q}}$ and $W=\mathfrak{a}_{\mathbb{Q}}$.

From this data we constructed in BHKRYa a smooth Deligne-Mumford stack $\operatorname{Sh}(G, \mathcal{D})$ of dimension $n-1$ over $\boldsymbol{k}$ with complex points

$$
\operatorname{Sh}(G, \mathcal{D})(\mathbb{C})=G(\mathbb{Q}) \backslash \mathcal{D} \times G\left(\mathbb{A}_{f}\right) / K .
$$

The reductive group $G \subset \mathrm{GU}\left(W_{0}\right) \times \mathrm{GU}(W)$ is the largest subgroup on which the two similitude characters agree, and $K \subset G\left(\mathbb{A}_{f}\right)$ is the largest subgroup stabilizing the $\widehat{\mathbb{Z}}$-lattices $\widehat{\mathfrak{a}}_{0} \subset W_{0}\left(\mathbb{A}_{f}\right)$ and $\widehat{\mathfrak{a}} \subset W\left(\mathbb{A}_{f}\right)$.

We also defined in [BHKRYa, §2.3] an integral model

$$
\mathcal{S}_{\mathrm{Kra}} \subset \mathcal{M}_{(1,0)} \times \mathcal{O}_{k} \mathcal{M}_{(n-1,1)}^{\mathrm{Kra}}
$$

of $\operatorname{Sh}(G, \mathcal{D})$. It is regular and flat over $\mathcal{O}_{\boldsymbol{k}}$, and admits a canonical toroidal compactification $\mathcal{S}_{\mathrm{Kra}} \hookrightarrow \mathcal{S}_{\mathrm{Kra}}^{*}$ whose boundary is a smooth divisor.

The main result of BHKRYa] is the construction of a formal generating series of arithmetic divisors

$$
\widehat{\phi}(\tau)=\sum_{m \geqslant 0} \hat{\mathcal{Z}}_{\mathrm{Kra}}^{\mathrm{total}}(m) \cdot q^{m} \in \widehat{\mathrm{Ch}}_{\mathbb{Q}}^{1}\left(\mathcal{S}_{\mathrm{Kra}}^{*}\right)[[q]]
$$

valued in the Gillet-Soulé codimension one arithmetic Chow group with rational coefficients, extended to allow log-log Green functions at the boundary as in BGKK07, BBGK07, and the proof that this generating series is modular of weight $n$, level $\Gamma_{0}(D)$, and character $\chi_{\boldsymbol{k}}^{n}$. The modularity result implies that the coefficients span a finite-dimensional subspace of the arithmetic Chow group [BHKRYa, Remark 7.1.2].

After passing to the arithmetic Chow group with complex coefficients, for any classical modular form

$$
g \in S_{n}\left(\Gamma_{0}(D), \chi_{\boldsymbol{k}}^{n}\right)
$$

we may form the Petersson inner product

$$
\langle\widehat{\phi}, g\rangle_{\mathrm{Pet}}=\int_{\Gamma_{0}(D) \backslash \mathcal{H}} \overline{g(\tau)} \cdot \widehat{\phi}(\tau) \frac{d u d v}{v^{2-n}}
$$

where $\tau=u+i v$. As in [Kud04], define the arithmetic theta lift

$$
\widehat{\theta}(g)=\langle\widehat{\phi}, g\rangle_{\mathrm{Pet}} \in \widehat{\mathrm{Ch}}_{\mathbb{C}}^{1}\left(\mathcal{S}_{\mathrm{Kra}}^{*}\right) .
$$

Armed with the construction of the arithmetic theta lift (1.1.3), we are now able to complete the program of [How12, How15, BHY15] to prove Gross-Zagier style formulas relating arithmetic intersections to derivatives of $L$-functions.

The Shimura variety $\mathcal{S}_{\text {Kra }}^{*}$ carries different families of codimension $n-1$ cycles constructed from complex multiplication points, and our results show that the arithmetic intersections of these families with arithmetic lifts are related to central derivatives of $L$-functions. 
1.2. Central derivatives and small $\mathbf{C M}$ points. In $\$ 2$ we construct an étale and proper Deligne-Mumford stack $\mathcal{Y}_{\text {sm }}$ over $\mathcal{O}_{\boldsymbol{k}}$, along with a morphism

$$
\mathcal{Y}_{\mathrm{sm}} \rightarrow \mathcal{S}_{\mathrm{Kra}}^{*}
$$

This is the small CM cycle. Intersecting arithmetic divisors against $\mathcal{Y}_{\mathrm{sm}}$ defines a linear functional

$$
\left[-: \mathcal{Y}_{\mathrm{sm}}\right]: \widehat{\mathrm{Ch}}_{\mathbb{C}}^{1}\left(\mathcal{S}_{\mathrm{Kra}}^{*}\right) \rightarrow \mathbb{C},
$$

and our first main result computes the image of the arithmetic theta lift (1.1.3) under this linear functional.

The statement involves the convolution $L$-function $L\left(\tilde{g}, \theta_{\Lambda}, s\right)$ of two modular forms

$$
\tilde{g} \in S_{n}\left(\bar{\omega}_{L}\right), \quad \theta_{\Lambda} \in M_{n-1}\left(\omega_{\Lambda}^{\vee}\right)
$$

valued in finite-dimensional representations of $\mathrm{SL}_{2}(\mathbb{Z})$. We refer the reader to 2.3 for the precise definitions. Here we note only that $\tilde{g}$ is the image of $g$ under an induction map

$$
S_{n}\left(\Gamma_{0}(D), \chi_{\boldsymbol{k}}^{n}\right) \rightarrow S_{n}\left(\bar{\omega}_{L}\right)
$$

from scalar-valued forms to vector-valued forms, that $\theta_{\Lambda}$ is the theta function attached to a quadratic space $\Lambda$ over $\mathbb{Z}$ of signature $(2 n-2,0)$, and that the $L$-function $L\left(\tilde{g}, \theta_{\Lambda}, s\right)$ vanishes at its center of symmetry $s=0$.

Theorem A. The arithmetic theta lift (1.1.3) satisfies

$$
\left[\hat{\theta}(g): \mathcal{Y}_{\mathrm{sm}}\right]=-\left.\operatorname{deg}_{\mathbb{C}}\left(\mathcal{Y}_{\mathrm{sm}}\right) \cdot \frac{d}{d s} L\left(\tilde{g}, \theta_{\Lambda}, s\right)\right|_{s=0} .
$$

Here we have defined

$$
\operatorname{deg}_{\mathbb{C}}\left(\mathcal{Y}_{\mathrm{sm}}\right)=\sum_{y \in \mathcal{Y}_{\mathrm{sm}}(\mathbb{C})} \frac{1}{|\operatorname{Aut}(y)|},
$$

where the sum is over the finitely many isomorphism classes of the groupoid of complex points of $\mathcal{Y}_{\mathrm{sm}}$, viewed as an $\mathcal{O}_{\boldsymbol{k}}$-stack.

The proof is given in $\$ 2$, by combining the modularity result of [BHKRYa] with the main result of [BHY15]. In 93 we provide alternative formulations of Theorem $\AA$ that involve the usual convolution $L$-function of scalar-valued modular forms, as opposed to the vector-valued forms $\tilde{g}$ and $\theta_{\Lambda}$. See especially Theorem 3.4.1.

1.3. Central derivatives and big CM points. Fix a totally real field $F$ of degree $n$, and define a CM field

$$
E=\boldsymbol{k} \otimes_{\mathbb{Q}} F .
$$

Let $\Phi \subset \operatorname{Hom}(E, \mathbb{C})$ be a CM type of signature $(n-1,1)$, in the sense that there is a unique $\varphi^{\mathrm{sp}} \in \Phi$, called the special embedding, whose restriction to 
$\boldsymbol{k}$ agrees with the complex conjugate of the inclusion $\boldsymbol{k} \subset \mathbb{C}$. The reflex field of the pair $(E, \Phi)$ is

$$
E_{\Phi}=\varphi^{\mathrm{sp}}(E) \subset \mathbb{C},
$$

and we denote by $\mathcal{O}_{\Phi} \subset E_{\Phi}$ its ring of integers.

We define in $\$ 4.2$ an étale and proper Deligne-Mumford stack $\mathcal{Y}_{\text {big }}$ over $\mathcal{O}_{\Phi}$, along with a morphism of $\mathcal{O}_{\boldsymbol{k}}$-stacks

$$
\mathcal{Y}_{\text {big }} \rightarrow \mathcal{S}_{\text {Kra }}^{*}
$$

This is the big CM cycle. Here we view $\mathcal{Y}_{\text {big }}$ as an $\mathcal{O}_{\boldsymbol{k}}$-stack using the inclusion $\mathcal{O}_{k} \subset \mathcal{O}_{\Phi}$ of subrings of $\mathbb{C}$ (which is the complex conjugate of the special embedding $\left.\varphi^{\mathrm{sp}}: \mathcal{O}_{\boldsymbol{k}} \rightarrow \mathcal{O}_{\Phi}\right)$. Intersecting arithmetic divisors against $\mathcal{Y}_{\text {big }}$ defines a linear functional

$$
\left[-: \mathcal{Y}_{\text {big }}\right]: \widehat{\mathrm{Ch}}_{\mathbb{C}}^{1}\left(\mathcal{S}_{\mathrm{Kra}}^{*}\right) \rightarrow \mathbb{C} .
$$

Our second main result relates the image of the arithmetic theta lift (1.1.3) under this linear functional to the central derivative of a generalized $L$-function defined as the Petersson inner product $\langle E(s), \tilde{g}\rangle_{\text {Pet }}$. The modular form $\tilde{g}(\tau)$ is, once again, the image of $g(\tau)$ under the induction map (1.2.1). The modular form $E(\tau, s)$ is defined as the restriction via the diagonal embedding $\mathcal{H} \rightarrow \mathcal{H}^{n}$ of a weight one Hilbert modular Eisenstein series valued in the space of the contragredient representation $\omega_{L}^{\vee}$. See $\$ 4.3$ for details.

Theorem B. Assume that the discriminants of $\boldsymbol{k} / \mathbb{Q}$ and $F / \mathbb{Q}$ are odd and relatively prime. The arithmetic theta lift (1.1.3) satisfies

$$
\left[\hat{\theta}(g): \mathcal{Y}_{\text {big }}\right]=\left.\frac{-1}{n} \cdot \operatorname{deg}_{\mathbb{C}}\left(\mathcal{Y}_{\text {big }}\right) \cdot \frac{d}{d s}\langle E(s), \tilde{g}\rangle_{\text {Pet }}\right|_{s=0} .
$$

Here we have defined

$$
\operatorname{deg}_{\mathbb{C}}\left(\mathcal{Y}_{\text {big }}\right)=\sum_{y \in \mathcal{Y}_{\text {big }}(\mathbb{C})} \frac{1}{|\operatorname{Aut}(y)|},
$$

where the sum is over the finitely many isomorphism classes of the groupoid of complex points of $\mathcal{Y}_{\mathrm{big}}$, viewed as an $\mathcal{O}_{\boldsymbol{k}}$-stack.

The proof is given in $\$ 4$, by combining the modularity result of [BHKRYa] with the intersection calculations of [BKY12, How12, How15].

1.4. Colmez's conjecture. Suppose $E$ is a CM field with maximal totally real subfield $F$. Let $D_{E}$ and $D_{F}$ be the absolute discriminants of $E$ and $F$, set $\Gamma_{\mathbb{R}}(s)=\pi^{-s / 2} \Gamma(s / 2)$, and define the completed $L$-function

$$
\Lambda\left(s, \chi_{E}\right)=\left|\frac{D_{E}}{D_{F}}\right|^{\frac{s}{2}} \Gamma_{\mathbb{R}}(s+1)^{[F: \mathbb{Q}]} L\left(s, \chi_{E}\right)
$$


of the character $\chi_{E}: \mathbb{A}_{F}^{\times} \rightarrow\{ \pm 1\}$ determined by $E / F$. It satisfies the functional equation $\Lambda\left(1-s, \chi_{E}\right)=\Lambda\left(s, \chi_{E}\right)$, and

$$
\frac{\Lambda^{\prime}\left(0, \chi_{E}\right)}{\Lambda\left(0, \chi_{E}\right)}=\frac{L^{\prime}\left(0, \chi_{E}\right)}{L\left(0, \chi_{E}\right)}+\frac{1}{2} \log \left|\frac{D_{E}}{D_{F}}\right|-\frac{[F: \mathbb{Q}]}{2} \log \left(4 \pi e^{\gamma}\right)
$$

where $\gamma=-\Gamma^{\prime}(1)$ is the Euler-Mascheroni constant.

Suppose $A$ is an abelian variety over $\mathbb{C}$ with complex multiplication by $\mathcal{O}_{E}$ and CM type $\Phi$. In particular $A$ is defined over the algebraic closure of $\mathbb{Q}$ in $\mathbb{C}$. It is a theorem of Colmez Col93] that the Faltings height

$$
h_{(E, \Phi)}^{\text {Falt }}=h^{\text {Falt }}(A)
$$

depends only on the pair $(E, \Phi)$, and not on $A$ itself. Moreover, Colmez gave a conjectural formula for this Faltings height in terms of logarithmic derivatives of Artin $L$-functions. In the special case where $E=\boldsymbol{k}$, Colmez's conjecture reduces to the well-known Chowla-Selberg formula

$$
h_{\boldsymbol{k}}^{\text {Falt }}=-\frac{1}{2} \cdot \frac{\Lambda^{\prime}\left(0, \chi_{\boldsymbol{k}}\right)}{\Lambda\left(0, \chi_{\boldsymbol{k}}\right)}-\frac{1}{4} \cdot \log \left(16 \pi^{3} e^{\gamma}\right),
$$

where we omit the CM type $\{\operatorname{id}\} \subset \operatorname{Hom}(\boldsymbol{k}, \mathbb{C})$ from the notation.

Now suppose we are in the special case of 1.3 , where

$$
E=\boldsymbol{k} \otimes_{\mathbb{Q}} F
$$

and $\Phi \subset \operatorname{Hom}(E, \mathbb{C})$ has signature $(n-1,1)$. In this case, Colmez's conjecture simplifies to the equality of the following theorem.

Theorem C ([YY18]). For a pair $(E, \Phi)$ as above,

$$
h_{(E, \Phi)}^{\text {Falt }}=-\frac{2}{n} \cdot \frac{\Lambda^{\prime}\left(0, \chi_{E}\right)}{\Lambda\left(0, \chi_{E}\right)}+\frac{4-n}{2} \cdot \frac{\Lambda^{\prime}\left(0, \chi_{\boldsymbol{k}}\right)}{\Lambda\left(0, \chi_{\boldsymbol{k}}\right)}-\frac{n}{4} \cdot \log \left(16 \pi^{3} e^{\gamma}\right) .
$$

In [BHKRYa, §2.4] we defined the line bundle of weight one modular forms $\boldsymbol{\omega}$ on $\mathcal{S}_{\mathrm{Kra}}^{*}$. It was endowed it with a hermitian metric in [BHKRYa, $\$ 7.2$ ], and the resulting metrized line bundle determines a class

$$
\widehat{\omega} \in \widehat{\mathrm{Ch}}_{\mathbb{Q}}^{1}\left(\mathcal{S}_{\mathrm{Kra}}^{*}\right) \text {. }
$$

The constant term of (1.1.2) is

$$
\widehat{\mathcal{Z}}_{\mathrm{Kra}}^{\mathrm{tot}}(0)=-\widehat{\boldsymbol{\omega}}+(\mathrm{Exc},-\log (D))
$$

where Exc is the exceptional locus of $\mathcal{S}_{\mathrm{Kra}}^{*}$ appearing in BHKRYa, Theorem 2.3.4]. It is a smooth effective Cartier divisor supported in characteristics dividing $D$, and we view it as an arithmetic divisor by endowing it with the constant Green function $-\log (D)$ in the complex fiber.

Theorem D. The metrized line bundle $\widehat{\boldsymbol{\omega}}$ satisfies

$$
\left[\widehat{\boldsymbol{\omega}}: \mathcal{Y}_{\text {big }}\right]=\frac{-2}{n} \cdot \operatorname{deg}_{\mathbb{C}}\left(\mathcal{Y}_{\text {big }}\right) \cdot \frac{\Lambda^{\prime}\left(0, \chi_{E}\right)}{\Lambda\left(0, \chi_{E}\right)} .
$$


Theorem $\mathrm{C}$ is proved in YY18 as a consequence of the average version of Colmez's conjecture AGHMP18, YZ18, How20. Note that the proof in YY18 does not require our standing hypothesis that $\operatorname{disc}(\boldsymbol{k})$ is odd. Of course the assumption that $\operatorname{disc}(\boldsymbol{k})$ is odd is still needed for Theorem D. as it is only under these hypotheses that we have even defined the integral model $\mathcal{S}_{\mathrm{Kra}}^{*}$ and its line bundle of weight one modular forms.

In $\$ 5$ we will show that Theorems $\mathrm{C}$ and $\mathrm{D}$ are equivalent. One can interpret this in one of two ways. As Theorem $\mathrm{C}$ is already known, this equivalence proves Theorem D. On the other hand, in 4.5 will give an independent proof of Theorem D under the additional assumption that the discriminants of $\boldsymbol{k}$ and $F$ are odd and relatively prime. In this way we obtain a new proof of Theorem $\mathrm{C}$ under these extra hypotheses.

1.5. The case $n=2$. Throughout the introduction we have assumed that $n \geqslant 3$, and the reader might wonder how much of what we have written extends to the case $n=2$.

As explained in [BHKRYa, §1.6], when $n=2$ the proof of the modularity of (1.1.2) breaks down because there is no known integral model of $\operatorname{Sh}(G, \mathcal{D})$ whose reduction at the primes of $\mathcal{O}_{\boldsymbol{k}}$ dividing $D$ is normal. The existence of such a model when $n>2$ is used in [loc. cit.] to compute the vertical components of divisors of Borcherds products.

When $n=2$, the Shimura variety $\operatorname{Sh}(G, \mathcal{D})$ is essentially a union of modular curves (if the $\boldsymbol{k}$-hermitian space $W$ admits an isotropic line) or compact quaternionic Shimura curves (if $W$ is anisotropic). In either case the analogues of Theorems $\mathrm{A}$ and $\mathrm{B}$ are close in spirit to the Gross-Zagier theorem GZ86 and its generalizations YYZ13. In particular, the statement of Theorems $\mathrm{A}$ is quite parallel to the key result Theorem 6.1 in [GZ86, Section 1.6]. If we interchange in the computation of $\left[\hat{\theta}(g): \mathcal{Y}_{\mathrm{sm}}\right]$ the order of taking the Petersson inner product and the height pairing, this quantity is very analogous to the left hand side of Theorem 6.1 in [GZ86]. Both quantities are expressed as central derivatives of a Rankin convolution $L$-function of $g$ and a binary theta function which is determined by the CM cycle in question. If $g$ is a newform, then $\hat{\theta}(g)$ should lie in a $g$-isotypical component and the height pairing in our Theorem A should be proportional to the height of the $g$-isotypical component of (a twist of) $\mathcal{Y}_{\mathrm{sm}}$. It would be interesting to make such a comparison precise. However, note that there are substantial differences as well. While we work with unitary Shimura varieties and CM points whose discriminants are equal to the level, Gross and Zagier work with $\mathrm{GL}_{2}$ Shimura varieties and CM points whose discriminants are coprime to the level.

Theorem $\mathrm{C}$ is true as stated when $n=2$, and is proved in [YY18. Indeed, Colmez's conjecture is known for all quartic CM fields. If the quartic CM field is Galois over $\mathbb{Q}$, then the Galois group is abelian and Colmez's conjecture is known by work of Colmez Col93 and Obus Obu13. In the non-Galois case the CM types form a single Aut $(\mathbb{C} / \mathbb{Q})$-orbit; as Colmez's 
conjecture is constant on such orbits, the full Colmez conjecture follows from the average case proved in AGHMP18, and YY18.

Theorem $\mathrm{D}$ is also true as stated when $n=2$. Indeed, when we prove the equivalence of Theorems $\mathrm{C}$ and $\mathrm{D}$ in $\$ 5$ we only assume $n \geqslant 2$.

1.6. Thanks. The results of this paper are the outcome of a long term project, begun initially in Bonn in June of 2013, and supported in a crucial way by three weeklong meetings at AIM, in Palo Alto (May of 2014) and San Jose (November of 2015 and 2016), as part of their AIM SQuaRE's program. The opportunity to spend these periods of intensely focused efforts on the problems involved was essential. We would like to thank the University of Bonn and AIM for their support.

\section{Small CM CyCles AND DeRivatives of $L$-FunCtions}

In this section we combine the results of [BHKRYa] and BHY15] to prove Theorem A, Although we will restrict to $n \geqslant 3$ in 2.5 , we allow $n \geqslant 2$ until that point.

2.1. A Shimura variety of dimension zero. Define a rank three torus $T_{\mathrm{sm}}$ over $\mathbb{Q}$ as the fiber product

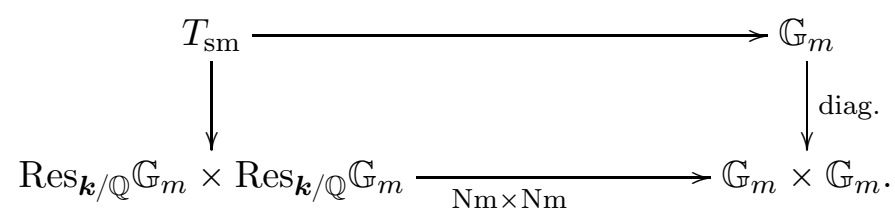

Its group of $\mathbb{Q}$-points is

$$
T_{\mathrm{sm}}(\mathbb{Q}) \cong\left\{(x, y) \in \boldsymbol{k}^{\times} \times \boldsymbol{k}^{\times}: x \bar{x}=y \bar{y}\right\} .
$$

The fixed embedding $\boldsymbol{k} \subset \mathbb{C}$ identifies Deligne's torus $\mathbb{S}$ with the real algebraic group $\left(\operatorname{Res}_{\boldsymbol{k} / \mathbb{Q}} \mathbb{G}_{m}\right){ }_{\mathbb{R}}$, and the diagonal inclusion

$$
\mathbb{S} \hookrightarrow\left(\operatorname{Res}_{\boldsymbol{k} / \mathbb{Q}} \mathbb{G}_{m}\right)_{\mathbb{R}} \times\left(\operatorname{Res}_{\boldsymbol{k} / \mathbb{Q}} \mathbb{G}_{m}\right)_{\mathbb{R}}
$$

factors through a morphism $h_{\mathrm{sm}}: \mathbb{S} \rightarrow T_{\mathrm{sm}, \mathbb{R}}$. The pair $\left(T_{\mathrm{sm}},\left\{h_{\mathrm{sm}}\right\}\right)$ is a Shimura datum, which, along with the compact open subgroup

$$
K_{\mathrm{sm}}=T_{\mathrm{sm}}\left(\mathbb{A}_{f}\right) \cap\left(\widehat{\mathcal{O}}_{\boldsymbol{k}}^{\times} \times \widehat{\mathcal{O}}_{\boldsymbol{k}}^{\times}\right),
$$

determines a 0 -dimensional $\boldsymbol{k}$-stack $\operatorname{Sh}\left(T_{\mathrm{sm}}\right)$ with complex points

$$
\operatorname{Sh}\left(T_{\mathrm{sm}}\right)(\mathbb{C})=T_{\mathrm{sm}}(\mathbb{Q}) \backslash\left\{h_{\mathrm{sm}}\right\} \times T_{\mathrm{sm}}\left(\mathbb{A}_{f}\right) / K_{\mathrm{sm}} .
$$

2.2. The small CM cycle. The Shimura variety just constructed has a moduli interpretation, which allows us to construct an integral model. The interpretation we have in mind requires first choosing a triple $\left(\mathfrak{a}_{0}, \mathfrak{a}_{1}, \mathfrak{b}\right)$ in which

- $\mathfrak{a}_{0}$ is a self-dual hermitian $\mathcal{O}_{\boldsymbol{k}}$-lattice of signature $(1,0)$,

- $\mathfrak{a}_{1}$ is a self-dual hermitian $\mathcal{O}_{\boldsymbol{k}}$-lattice of signature $(0,1)$,

- $\mathfrak{b}$ is a self-dual hermitian $\mathcal{O}_{\boldsymbol{k}}$-lattice of signature $(n-1,0)$. 
The hermitian forms on $\mathfrak{a}_{0}$ and $\mathfrak{b}$ induce a hermitian form of signature $(n-1,0)$ on the projective $\mathcal{O}_{\boldsymbol{k}}$-module

$$
\Lambda=\operatorname{Hom}_{\mathcal{O}_{\boldsymbol{k}}}\left(\mathfrak{a}_{0}, \mathfrak{b}\right),
$$

as explained in [BHY15, §2.1] or [BHKRYa, (2.1.5)].

Recall from [BHY15, §3.1] or [BHKRYa, §2.3] the $\mathcal{O}_{\boldsymbol{k}}$-stacks $\mathcal{M}_{(p, 0)}$ and $\mathcal{M}_{(0, p)}$. Both parametrize abelian schemes $A \rightarrow S$ of relative dimension $p \geqslant$ 1 over $\mathcal{O}_{\boldsymbol{k}}$-schemes, endowed with principal polarizations and $\mathcal{O}_{\boldsymbol{k}}$-actions. For the first moduli problem we impose the signature $(p, 0)$ condition that $\mathcal{O}_{\boldsymbol{k}}$ acts on the $\mathcal{O}_{S}$-module $\operatorname{Lie}(A)$ via the structure morphism $\mathcal{O}_{\boldsymbol{k}} \rightarrow \mathcal{O}_{S}$. For the second we impose the signature $(0, p)$ condition that the action is by the complex conjugate of the structure morphism. Both of these stacks are étale and proper over $\mathcal{O}_{\boldsymbol{k}}$ by [How15, Proposition 2.1.2].

Remark 2.2.1. The generic fibers of $\mathcal{M}_{(1,0)}$ and $\mathcal{M}_{(0,1)}$ are the Shimura varieties associated to $\mathfrak{a}_{0 \mathbb{Q}}$ and $\mathfrak{a}_{1 \mathbb{Q}}$, while the generic fiber of $\mathcal{M}_{(n-1,0)}$ contains the Shimura variety associated to $\mathfrak{b}_{\mathbb{Q}}$ as an open and closed substack. For more precise information, see [KR14, Proposition 2.13] and the lemma that precedes it.

Denote by $\tilde{\mathcal{Y}}_{\mathrm{sm}}$ the functor that associates to every $\mathcal{O}_{\boldsymbol{k}}$-scheme $S$ the groupoid of quadruples $\left(A_{0}, A_{1}, B, \eta\right)$ in which

$$
\left(A_{0}, A_{1}, B\right) \in \mathcal{M}_{(1,0)}(S) \times \mathcal{M}_{(0,1)}(S) \times \mathcal{M}_{(n-1,0)}(S),
$$

and

$$
\eta: \underline{\operatorname{Hom}}_{\mathcal{O}_{k}}\left(A_{0}, B\right) \cong \underline{\Lambda}
$$

is an isomorphism of étale sheaves of hermitian $\mathcal{O}_{\boldsymbol{k}}$-modules, where the hermitian form on the left hand side is defined as in BHKRYa, (2.5.1)]. We impose the further condition that for every geometric point $s \rightarrow S$, and every prime $\ell \neq \operatorname{char}(s)$, there is an isomorphism of hermitian $\mathcal{O}_{\boldsymbol{k}, \ell}$-lattices

$$
\operatorname{Hom}_{\mathcal{O}_{\boldsymbol{k}}}\left(A_{0 s}\left[\ell^{\infty}\right], A_{1 s}\left[\ell^{\infty}\right]\right) \cong \operatorname{Hom}_{\mathcal{O}_{\boldsymbol{k}}}\left(\mathfrak{a}_{0}, \mathfrak{a}_{1}\right) \otimes_{\mathbb{Z}} \mathbb{Z}_{\ell}
$$

\section{Lemma 2.2.2. If}

$$
s \rightarrow \mathcal{M}_{(1,0)} \times{ }_{\mathcal{O}_{k}} \mathcal{M}_{(0,1)} \times{ }_{\mathcal{O}_{k}} \mathcal{M}_{(n-1,0)}
$$

is a geometric point of characteristic 0 such that (2.2.3) holds for all primes $\ell$ except possibly one, then it holds for the remaining prime as well.

Proof. The proof is identical to [BHKRYa, Lemma 2.2.2].

Proposition 2.2.3. The functor $\tilde{\mathcal{Y}}_{\mathrm{sm}}$ is represented by a Deligne-Mumford stack, étale and proper over $\mathcal{O}_{\boldsymbol{k}}$, and there is a canonical isomorphism of $\boldsymbol{k}$-stacks

$$
\operatorname{Sh}\left(T_{\mathrm{sm}}\right) \cong \tilde{\mathcal{Y}}_{\mathrm{sm} / \boldsymbol{k}}
$$


Proof. For any $\mathcal{O}_{\boldsymbol{k}^{\text {-scheme }} S} S$, let $\mathcal{N}(S)$ be the groupoid of triples (2.2.1) satisfying (2.2.3) for every geometric point $s \rightarrow S$ and every prime $\ell \neq$ $\operatorname{char}(s)$. In other words, the definition is the same as $\tilde{\mathcal{Y}}_{\text {sm }}$ except that we omit the datum (2.2.2) from the moduli problem.

We interrupt the proof of Proposition 2.2 .3 for a lemma.

Lemma 2.2.4. The functor $\mathcal{N}$ is represented by an open and closed substack

$$
\mathcal{N} \subset \mathcal{M}_{(1,0)} \times{ }_{\mathcal{O}_{k}} \mathcal{M}_{(0,1)} \times{ }_{\mathcal{O}_{k}} \mathcal{M}_{(n-1,0)} .
$$

Proof. This is [BHY15, Proposition 5.2]. As the proof there is left to the reader, we indicate the idea. Let

$$
\mathcal{B} \subset \mathcal{M}_{(1,0)} \times{ }_{\mathcal{O}_{k}} \mathcal{M}_{(0,1)} \times_{\mathcal{O}_{k}} \mathcal{M}_{(n-1,0)}
$$

be one connected component, and suppose there is a geometric point $s \rightarrow \mathcal{B}$ of characteristic $p$ such that (2.2.3) holds for all $\ell \neq p$. The geometric fibers of the $\ell$-adic sheaf $\underline{\operatorname{Hom}}_{\mathcal{O}_{k}}\left(A_{0}\left[\ell^{\infty}\right], A_{1}\left[\ell^{\infty}\right]\right)$ on

$$
\mathcal{B}_{(p)}=\mathcal{B} \times_{\operatorname{Spec}(\mathbb{Z})} \operatorname{Spec}\left(\mathbb{Z}_{(p)}\right)
$$

are all isomorphic, and therefore (2.2.3) holds for all geometric points $s \rightarrow$ $\mathcal{B}_{(p)}$ and all $\ell \neq p$. In particular, using Lemma 2.2.2, if $s \rightarrow \mathcal{B}$ is a geometric point of characteristic 0 , then (2.2.3) holds for every prime $\ell$. Having proved this, one can reverse the argument to see that (2.2.3) holds for every geometric point $s \rightarrow \mathcal{B}$ and every $\ell \neq \operatorname{char}(s)$. Thus if the condition (2.2.3) holds at one geometric point, it holds at all geometric points on the same connected component.

We now return to the proof of Proposition 2.2.3. As noted above, the stacks $\mathcal{M}_{(p, 0)}$ and $\mathcal{M}_{(0, p)}$ are étale and proper over $\mathcal{O}_{\boldsymbol{k}}$, and hence the same is true of $\mathcal{N}$.

Let $\left(A_{0}, A_{1}, B\right)$ be the universal object over $\mathcal{N}$. Combining BHY15, Theorem 5.1] and [Hid04, Corollary 6.9], the étale sheaf $\underline{\operatorname{Hom}}_{\mathcal{O}_{\boldsymbol{k}}}\left(A_{0}, B\right)$ is represented by a Deligne-Mumford stack whose connected components are finite étale over $\mathcal{N}$. Fixing a geometric point $s \rightarrow \mathcal{N}$, we obtain a representation of $\pi_{1}^{e t}(\mathcal{N}, s)$ on a finitely generated $\mathcal{O}_{\boldsymbol{k}}$-module $\operatorname{Hom}_{\mathcal{O}_{\boldsymbol{k}}}\left(A_{0 s}, B_{s}\right)$, and the kernel of this representation cuts out a finite étale cover $\mathcal{N}^{\prime} \rightarrow \mathcal{N}$ over which the sheaf $\underline{\operatorname{Hom}}_{\mathcal{O}_{\boldsymbol{k}}}\left(A_{0}, B\right)$ becomes constant.

It is now easy to see that the functor $\tilde{\mathcal{Y}}_{\text {sm }}$ is represented by the disjoint union of finitely many copies of the maximal open and closed substack of $\mathcal{N}^{\prime}$ over which there exists an isomorphism (2.2.2).

It remains to construct the isomorphism (2.2.4). The natural actions of $\mathcal{O}_{\boldsymbol{k}}$ on $\mathfrak{a}_{0}$ and $\mathfrak{b}$, along with the complex conjugate of the natural action of $\mathcal{O}_{\boldsymbol{k}}$ on $\mathfrak{a}_{1}$, determine a morphism of reductive groups

$$
\operatorname{Res}_{\boldsymbol{k} / \mathbb{Q}} \mathbb{G}_{m} \times \operatorname{Res}_{\boldsymbol{k} / \mathbb{Q}} \mathbb{G}_{m} \stackrel{(w, z) \mapsto(w, \bar{z}, z)}{\longrightarrow} \mathrm{GU}\left(\mathfrak{a}_{0 \mathbb{Q}}\right) \times \mathrm{GU}\left(\mathfrak{a}_{1 \mathbb{Q}}\right) \times \mathrm{GU}\left(\mathfrak{b}_{\mathbb{Q}}\right) .
$$

Restricting this morphism to the subtorus $T_{\mathrm{sm}}$ defines a morphism

$$
\mathbb{S} \stackrel{h_{\mathrm{sm}}}{\longrightarrow} T_{\mathrm{sm}, \mathbb{R}} \rightarrow \mathrm{GU}\left(\mathfrak{a}_{0 \mathbb{R}}\right) \times \mathrm{GU}\left(\mathfrak{a}_{1 \mathbb{R}}\right) \times \mathrm{GU}\left(\mathfrak{b}_{\mathbb{R}}\right),
$$


endowing the real vector spaces $\mathfrak{a}_{0 \mathbb{R}}, \mathfrak{a}_{1 \mathbb{R}}$, and $\mathfrak{b}_{\mathbb{R}}$ with complex structures.

The isomorphism (2.2.4) on complex points sends a pair

$$
\left(h_{\mathrm{sm}}, g\right) \in \operatorname{Sh}\left(T_{\mathrm{sm}}\right)(\mathbb{C})
$$

to the quadruple $\left(A_{0}, A_{1}, B, \eta\right)$ defined by

$$
A_{0}(\mathbb{C})=\mathfrak{a}_{0 \mathbb{R}} / g \mathfrak{a}_{0}, \quad A_{1}(\mathbb{C})=\mathfrak{a}_{1 \mathbb{R}} / g \mathfrak{a}_{1}, \quad B(\mathbb{C})=\mathfrak{b}_{\mathbb{R}} / g \mathfrak{b},
$$

endowed with their natural $\mathcal{O}_{\boldsymbol{k}}$-actions and polarizations as in the proof of BHKRYa, Proposition 2.2.1]. The datum $\eta$ is the canonical identification

$$
\operatorname{Hom}_{\mathcal{O}_{\boldsymbol{k}}}\left(A_{0}, B\right)=\operatorname{Hom}_{\mathcal{O}_{\boldsymbol{k}}}\left(g \mathfrak{a}_{0}, g \mathfrak{b}\right)=\operatorname{Hom}_{\mathcal{O}_{\boldsymbol{k}}}\left(\mathfrak{a}_{0}, \mathfrak{b}\right)=\Lambda .
$$

It follows from the theory of canonical models that this isomorphism on complex points descends to an isomorphism of $\boldsymbol{k}$-stacks, completing the proof of Proposition 2.2.3.

The finite group $\operatorname{Aut}(\Lambda)$ of automorphisms of the hermitian lattice $\Lambda$ acts on $\widetilde{\mathcal{Y}}_{\text {sm }}$ by

$$
\gamma *\left(A_{0}, A_{1}, B, \eta\right)=\left(A_{0}, A_{1}, B, \gamma \circ \eta\right),
$$

allowing us to form the stack quotient $\mathcal{Y}_{\text {sm }}=\operatorname{Aut}(\Lambda) \backslash \widetilde{\mathcal{Y}}_{\mathrm{sm}}$. The forgetful map

$$
\tilde{\mathcal{Y}}_{\text {sm }} \rightarrow \mathcal{M}_{(1,0)} \times \mathcal{M}_{(0,1)} \times \mathcal{M}_{(n-1,0)}
$$

(all fiber products over $\mathcal{O}_{\boldsymbol{k}}$ ) factors through an open and closed immersion

$$
\mathcal{Y}_{\mathrm{sm}} \rightarrow \mathcal{M}_{(1,0)} \times \mathcal{M}_{(0,1)} \times \mathcal{M}_{(n-1,0)}
$$

whose image is the open and closed substack $\mathcal{N}$ of Lemma 2.2.4.

The triple $\left(\mathfrak{a}_{0}, \mathfrak{a}_{1}, \mathfrak{b}\right)$ determines a pair $\left(\mathfrak{a}_{0}, \mathfrak{a}\right)$ as in the introduction, simply by setting $\mathfrak{a}=\mathfrak{a}_{1} \oplus \mathfrak{b}$. This data determines a unitary Shimura variety with integral model $\mathcal{S}_{\mathrm{Kra}}$ as in (1.1.1), and there is a commutative diagram

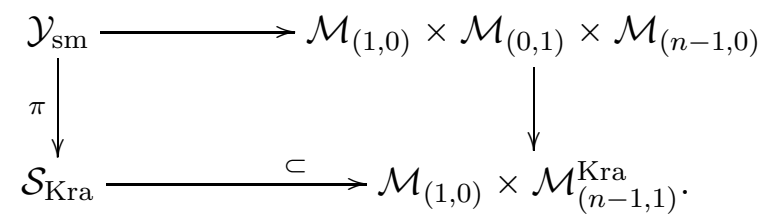

The vertical arrow on the right sends

$$
\left(A_{0}, A_{1}, B\right) \mapsto\left(A_{0}, A_{1} \times B\right),
$$

and the arrow $\pi$ is defined by the commutativity of the diagram.

Remark 2.2.5. In order for $A_{1} \times B$ to define a point of $\mathcal{M}_{(n-1,1)}^{\mathrm{Kra}}$, we must endow its Lie algebra with a codimension one subsheaf

$$
\mathcal{F}_{A_{1} \times B} \subset \operatorname{Lie}\left(A_{1} \times B\right)
$$

satisfying Krämer's condition [BHKRYa, $\S 2.3]$. We choose $\mathcal{F}_{A_{1} \times B}=\operatorname{Lie}(B)$. 
Definition 2.2.6. Composing the morphism $\pi$ in the diagram above with the inclusion of $\mathcal{S}_{\mathrm{Kra}}$ into its toroidal compactification, we obtain a morphism of $\mathcal{O}_{\boldsymbol{k}}$-stacks

called the small CM cycle.

$$
\pi: \mathcal{Y}_{\mathrm{sm}} \rightarrow \mathcal{S}_{\mathrm{Kra}}^{*}
$$

As in How15, Definition 3.1.8], there is a linear functional

$$
\widehat{\mathrm{Ch}}_{\mathbb{C}}^{1}\left(\mathcal{S}_{\mathrm{Kra}}^{*}\right) \rightarrow \mathbb{C}
$$

called arithmetic degree along $\mathcal{Y}_{\text {sm }}$ and denoted $\widehat{\mathcal{Z}} \mapsto\left[\widehat{\mathcal{Z}}: \mathcal{Y}_{\text {sm }}\right]$, defined as the composition

$$
\widehat{\mathrm{Ch}}_{\mathbb{C}}^{1}\left(\mathcal{S}_{\mathrm{Kra}}^{*}\right) \stackrel{\pi^{*}}{\longrightarrow} \widehat{\mathrm{Ch}}_{\mathbb{C}}^{1}\left(\mathcal{Y}_{\mathrm{sm}}\right) \stackrel{\widehat{\mathrm{deg}}}{\longrightarrow} \mathbb{C}
$$

The first arrow is pullback of arithmetic divisors. The second arrow (arithmetic degree) is normalized as follows: An irreducible divisor $\mathcal{Z} \subset \mathcal{Y}_{\text {sm }}$ is necessarily supported in finitely many nonzero characteristics, and hence any $\mathbb{C}$-valued function $\operatorname{Gr}(\mathcal{Z}, \cdot)$ on the finite set $\mathcal{Y}_{\text {sm }}(\mathbb{C})$ defines a Green function for it. The arithmetic degree of the arithmetic divisor

$$
(\mathcal{Z}, \operatorname{Gr}(\mathcal{Z}, \cdot)) \in \widehat{\operatorname{Ch}}_{\mathbb{C}}^{1}\left(\mathcal{Y}_{\text {sm }}\right)
$$

is defined to be

$$
\widehat{\operatorname{deg}}(\mathcal{Z}, \operatorname{Gr}(\mathcal{Z}, \cdot))=\sum_{\mathfrak{q} \subset \mathcal{O}_{k}} \sum_{z \in \mathcal{Z}\left(\mathbb{F}_{\mathfrak{q}}^{\text {alg }}\right)} \frac{\log (\mathrm{N}(\mathfrak{q}))}{\# \operatorname{Aut} \mathcal{X}(z)}+\sum_{z \in \mathcal{Y}_{\mathrm{sm}}(\mathbb{C})} \frac{\operatorname{Gr}(\mathcal{Z}, z)}{\# \operatorname{Aut}_{\mathcal{Y}_{\mathrm{sm}}(\mathbb{C})}(z)},
$$

where $\mathbb{F}_{\mathfrak{q}}^{\text {alg }}$ is an algebraic closure of $\mathcal{O}_{\boldsymbol{k}} / \mathfrak{q}$, and $\mathrm{N}(\mathfrak{q})=\#\left(\mathcal{O}_{\boldsymbol{k}} / \mathfrak{q}\right)$.

Remark 2.2.7. The above definition of arithmetic degree does not include a factor of $1 / 2$ in front of the archimedean contribution, seemingly in disagreement with the usual definition (see GS90, §3.4.3] for example). In fact there is no disagreement. Our convention is that $\mathcal{Y}_{\mathrm{sm}}(\mathbb{C})$ means the complex points of $\mathcal{Y}_{\text {sm }}(\mathbb{C})$ as a $\boldsymbol{k}$-stack, whereas in the usual definition it would be regarded as a $\mathbb{Q}$-stack. Thus the usual definition includes a sum over twice as many complex points, but with a $1 / 2$ in front.

Remark 2.2.8. The small CM cycle arises from a morphism of Shimura varieties. Indeed, there is a morphism of Shimura data $\left(T_{\mathrm{sm}},\left\{h_{\mathrm{sm}}\right\}\right) \rightarrow$ $(G, \mathcal{D})$, and the induced morphism of Shimura varieties sits in a commutative diagram

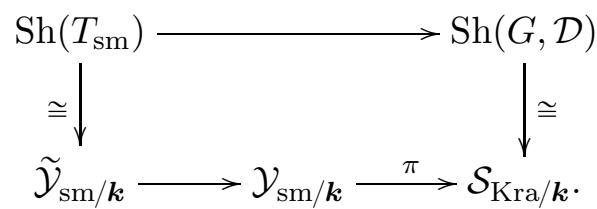

Proposition 2.2.9. The degree $\operatorname{deg}_{\mathbb{C}}\left(\mathcal{Y}_{\mathrm{sm}}\right)$ of Theorem $\triangle$ satisfies

$$
\operatorname{deg}_{\mathbb{C}}\left(\mathcal{Y}_{\mathrm{sm}}\right)=\left(h_{\boldsymbol{k}} / w_{\boldsymbol{k}}\right)^{2} \cdot \frac{2^{1-o(D)}}{|\operatorname{Aut}(\Lambda)|},
$$


where $o(D)$ is the number of distinct prime divisors of $D$.

Proof. This is an elementary calculation. Briefly, the groupoid $\mathcal{Y}_{\mathrm{sm}}(\mathbb{C})$ has $2^{1-o(D)} h_{\boldsymbol{k}}^{2}$ isomorphism classes of points, and each point has the same automorphism group $\mathcal{O}_{\boldsymbol{k}}^{\times} \times \mathcal{O}_{\boldsymbol{k}}^{\times} \times U(\Lambda)$.

Recall from (1.4.2) that the constant term of (1.1.2) is

$$
\hat{\mathcal{Z}}_{\mathrm{Kra}}^{\text {tot }}(0)=-\widehat{\boldsymbol{\omega}}+(\operatorname{Exc},-\log (D))
$$

where $\hat{\boldsymbol{\omega}}$ is the metrized line bundle of weight one modular forms. The exceptional locus Exc $\subset \mathcal{S}_{\text {Kra }}$ was defined in [BHKRYa, §2.3]. It is a reduced effective Cartier divisor supported in characteristics dividing $D$, and can be characterized as follows. The integral model $\mathcal{S}_{\text {Kra }}$ carries over it an abelian scheme $A \rightarrow \mathcal{S}_{\mathrm{Kra}}$ of relative dimension $n$ endowed with an action of $\mathcal{O}_{\boldsymbol{k}}$. This abelian scheme is obtained by pulling back the universal object from the second factor of the fiber product in (1.1.1). If we let $\delta \in \mathcal{O}_{\boldsymbol{k}}$ be a fixed square root of $-D$, then Exc is the reduced stack underlying closed substack of $\mathcal{S}_{\mathrm{Kra}}$ defined by $\delta \cdot \operatorname{Lie}(A)=0$.

Proposition 2.2.10. The constant term (1.4.2) satisfies

$$
\left[\hat{\mathcal{Z}}_{\mathrm{Kra}}^{\mathrm{tot}}(0): \mathcal{Y}_{\mathrm{sm}}\right]=-\left[\widehat{\boldsymbol{\omega}}: \mathcal{Y}_{\mathrm{sm}}\right]=2 \operatorname{deg}_{\mathbb{C}}\left(\mathcal{Y}_{\mathrm{sm}}\right) \cdot \frac{\Lambda^{\prime}\left(0, \chi_{\boldsymbol{k}}\right)}{\Lambda\left(0, \chi_{\boldsymbol{k}}\right)}
$$

Proof. The second equality was proved in the course of proving BHY15, Theorem 6.4]. We note that the argument uses the Chowla-Selberg formula (1.4.1) in an essential way.

The first equality is equivalent to

$$
\left[(\operatorname{Exc},-\log (D)): \mathcal{Y}_{\mathrm{sm}}\right]=0
$$

and so it suffices to prove

$$
\left[(0, \log (D)): \mathcal{Y}_{\mathrm{sm}}\right]=\operatorname{deg}_{\mathbb{C}}\left(\mathcal{Y}_{\mathrm{sm}}\right) \cdot \log (D)=\left[(\operatorname{Exc}, 0): \mathcal{Y}_{\mathrm{sm}}\right]
$$

The first equality in (2.2.5) is obvious from the definitions. To prove the second equality, we first prove

$$
\mathcal{Y}_{\mathrm{sm}} \times \mathcal{S}_{\mathrm{Kra}} \operatorname{Exc}=\mathcal{Y}_{\mathrm{sm}} \times \operatorname{Spec}\left(\mathcal{O}_{\boldsymbol{k}}\right) \operatorname{Spec}\left(\mathcal{O}_{\boldsymbol{k}} / \mathfrak{d}_{\boldsymbol{k}}\right) .
$$

As the exceptional locus Exc $\subset \mathcal{S}_{\mathrm{Kra}}$ is reduced and supported in characteristics dividing $D$, it satisfies

$$
\operatorname{Exc} \subset \mathcal{S}_{\text {Kra }} \times \operatorname{Spec}\left(\mathcal{O}_{\boldsymbol{k}}\right) \operatorname{Spec}\left(\mathcal{O}_{\boldsymbol{k}} / \mathfrak{d}_{\boldsymbol{k}}\right) .
$$

This implies the inclusion $\subset$ in (2.2.6). As $\mathcal{Y}_{\text {sm }}$ is étale over $\mathcal{O}_{\boldsymbol{k}}$, the right hand side of (2.2.6) is reduced, and hence so is the left hand side. To prove that equality holds in (2.2.6), it now suffices to check the inclusion $\supset$ on the level of geometric points.

As above, let $\delta \in \mathcal{O}_{\boldsymbol{k}}$ be a square root of $-D$. Suppose $p \mid D$ is a prime, $\mathfrak{p} \subset \mathcal{O}_{k}$ is the unique prime above it, and $\mathbb{F}_{\mathfrak{p}}^{\text {alg }}$ is an algebraic closure of its residue field. Suppose we have a point $y \in \mathcal{Y}_{\text {sm }}\left(\mathbb{F}_{\mathfrak{p}}^{\text {alg }}\right)$ corresponding to a 
triple $\left(A_{0}, A_{1}, B\right)$ over $\mathbb{F}_{\mathfrak{p}}^{\text {alg }}$. As $\delta=0$ in $\mathbb{F}_{\mathfrak{p}}^{\text {alg }}$, the signature conditions imply that the endomorphism $\delta \in \mathcal{O}_{\boldsymbol{k}}$ kills the Lie algebras of $A_{0}, A_{1}$, and $B$. In particular $\delta$ kills the Lie algebra of $A_{1} \times B$, which is the pullback via

$$
\pi: \mathcal{Y}_{\mathrm{sm}} \rightarrow \mathcal{S}_{\mathrm{Kra}}
$$

of the universal $A \rightarrow \mathcal{S}_{\mathrm{Kra}}$. Using the characterization of Exc recalled above, we find that that $\pi(y) \in$ Exc. This proves (2.2.6).

The equality (2.2.6), and the fact that both sides of that equality are reduced, implies that

$$
\left[(\mathrm{Exc}, 0): \mathcal{Y}_{\mathrm{sm}}\right]=\sum_{p \mid D} \log (p) \sum_{y \in \mathcal{Y}_{\mathrm{sm}}\left(\mathbb{F}_{\mathfrak{p}}^{\mathrm{alg}}\right)} \frac{1}{|\operatorname{Aut}(y)|} .
$$

On the other hand, the étaleness of $\mathcal{Y}_{\mathrm{sm}} \rightarrow \operatorname{Spec}\left(\mathcal{O}_{\boldsymbol{k}}\right)$ implies that the right hand side is equal to

$$
\sum_{p \mid D} \log (p) \sum_{y \in \mathcal{Y}_{\mathrm{sm}}(\mathbb{C})} \frac{1}{|\operatorname{Aut}(y)|}=\log (D) \cdot \operatorname{deg}_{\mathbb{C}}\left(\mathcal{Y}_{\mathrm{sm}}\right),
$$

completing the proof of the second equality in (2.2.5).

2.3. The convolution $L$-function. Recall that we have defined a hermitian $\mathcal{O}_{\boldsymbol{k}}$-lattice $\Lambda=\operatorname{Hom}_{\mathcal{O}_{\boldsymbol{k}}}\left(\mathfrak{a}_{0}, \mathfrak{b}\right)$ of signature $(n-1,0)$. We also define hermitian $\mathcal{O}_{\boldsymbol{k}}$-lattices

$$
L_{0}=\operatorname{Hom}_{\mathcal{O}_{\boldsymbol{k}}}\left(\mathfrak{a}_{0}, \mathfrak{a}_{1}\right), \quad L=\operatorname{Hom}_{\mathcal{O}_{\boldsymbol{k}}}\left(\mathfrak{a}_{0}, \mathfrak{a}\right),
$$

of signature $(1,0)$ and $(n-1,1)$, so that $L \cong L_{0} \oplus \Lambda$.

The hermitian form $\langle\cdot, \cdot\rangle: L \times L \rightarrow \mathcal{O}_{k}$ determines a $\mathbb{Z}$-valued quadratic form $Q(x)=\langle x, x\rangle$ on $L$, and we denote in the same way its restrictions to $L_{0}$ and $\Lambda$. The dual lattice of $L$ with respect to the $\mathbb{Z}$-bilinear form

$$
\left[x_{1}, x_{2}\right]=Q\left(x_{1}+x_{2}\right)-Q\left(x_{1}\right)-Q\left(x_{2}\right)
$$

is $L^{\prime}=\mathfrak{d}_{k}^{-1} L$.

As in [BHY15, $\S 2.2]$ we denote by $S_{L}=\mathbb{C}\left[L^{\prime} / L\right]$ the space of complexvalued functions on $L^{\prime} / L$, and by $\omega_{L}: \mathrm{SL}_{2}(\mathbb{Z}) \rightarrow \operatorname{Aut}_{\mathbb{C}}\left(S_{L}\right)$ the Weil representation. There is a complex conjugate representation $\bar{\omega}_{L}$ on $S_{L}$ defined by

$$
\bar{\omega}_{L}(\gamma) \phi=\overline{\omega_{L}(\gamma) \bar{\phi}}
$$

Suppose we begin with a classical scalar-valued cusp form

$$
g(\tau)=\sum_{m>0} c(m) q^{m} \in S_{n}\left(\Gamma_{0}(D), \chi_{\boldsymbol{k}}^{n}\right)
$$

Such a form determines a vector-valued form

$$
\tilde{g}(\tau)=\sum_{\gamma \in \Gamma_{0}(D) \backslash \mathrm{SL}_{2}(\mathbb{Z})}\left(\left.g\right|_{n} \gamma\right)(\tau) \cdot \overline{\omega_{L}\left(\gamma^{-1}\right) \phi_{0}} \in S_{n}\left(\bar{\omega}_{L}\right),
$$


where $\phi_{0} \in S_{L}$ is the characteristic function of the trivial coset. This construction defines the induction map (1.2.1). The form $\tilde{g}(\tau)$ has a $q$-expansion

$$
\tilde{g}(\tau)=\sum_{m>0} \tilde{c}(m) q^{m}
$$

with coefficients $\tilde{c}(m) \in S_{L}$.

There is a similar Weil representation $\omega_{\Lambda}: \operatorname{SL}_{2}(\mathbb{Z}) \rightarrow \operatorname{Aut}_{\mathbb{C}}\left(S_{\Lambda}\right)$, and for every $m \in \mathbb{Q}$ we define a linear functional $R_{\Lambda}(m) \in S_{\Lambda}^{\vee}$ by

$$
R_{\Lambda}(m)(\phi)=\sum_{\substack{x \in \Lambda^{\prime} \\\langle x, x\rangle=m}} \phi(x)
$$

where $\phi \in S_{\Lambda}$ and $\langle\cdot, \cdot\rangle: \Lambda_{\mathbb{Q}} \times \Lambda_{\mathbb{Q}} \rightarrow \boldsymbol{k}$ is the $\mathbb{Q}$-linear extension of the hermitian form on $\Lambda$. The theta series

$$
\theta_{\Lambda}(\tau)=\sum_{m \in \mathbb{Q}} R_{\Lambda}(m) q^{m} \in M_{n-1}\left(\omega_{\Lambda}^{\vee}\right)
$$

is a modular form valued in the contragredient representation $S_{\Lambda}^{\vee}$.

As in [BHY15, §5.3] or [BY09, §4.4], we define the Rankin-Selberg convolution L-function

$$
L\left(\tilde{g}, \theta_{\Lambda}, s\right)=\Gamma\left(\frac{s}{2}+n-1\right) \sum_{m \geqslant 0} \frac{\left\{\overline{\tilde{c}(m)}, R_{\Lambda}(m)\right\}}{(4 \pi m)^{\frac{s}{2}+n-1}} .
$$

Here $\{\cdot, \cdot\}: S_{L} \times S_{L}^{\vee} \rightarrow \mathbb{C}$ is the tautological pairing. The inclusion

$$
\Lambda^{\prime} / \Lambda \rightarrow L^{\prime} / L
$$

induces a linear map $S_{L} \rightarrow S_{\Lambda}$ by restriction of functions, and we use the dual $S_{\Lambda}^{\vee} \rightarrow S_{L}^{\vee}$ to view $R_{\Lambda}(m)$ as an element of $S_{L}^{\vee}$.

Remark 2.3.1. The convolution $L$-function satisfies a functional equation in $s \mapsto-s$, forcing $L\left(\tilde{g}, \theta_{\Lambda}, 0\right)=0$.

Remark 2.3.2. In this generality, neither the cusp form $g$ nor the theta series $\theta_{\Lambda}$ is a Hecke eigenform. Thus the convolution $L$-function (2.3.3) cannot be expected to have an Euler product expansion.

2.4. A preliminary central derivative formula. We now recall the main result of [BHY15], and explain the connection between the cycles and Shimura varieties here and in that work.

Define hermitian $\widehat{\mathcal{O}}_{\boldsymbol{k}}$-lattices

$$
\mathbb{L}_{0, f}=\operatorname{Hom}_{\mathcal{O}_{\boldsymbol{k}}}\left(\mathfrak{a}_{0}, \mathfrak{a}_{1}\right) \otimes_{\mathbb{Z}} \widehat{\mathbb{Z}}, \quad \mathbb{L}_{f}=\operatorname{Hom}_{\mathcal{O}_{\boldsymbol{k}}}\left(\mathfrak{a}_{0}, \mathfrak{a}\right) \otimes_{\mathbb{Z}} \widehat{\mathbb{Z}},
$$

and let $\mathbb{L}_{0, \infty}$ and $\mathbb{L}_{\infty}$ be $\boldsymbol{k}_{\mathbb{R}}$-hermitian spaces of signatures $(1,0)$ and $(n, 0)$, respectively. In the terminology of [BHY15, §2.1], the pairs

$$
\mathbb{L}_{0}=\left(\mathbb{L}_{0, \infty}, \mathbb{L}_{0, f}\right), \quad \mathbb{L}=\left(\mathbb{L}_{\infty}, \mathbb{L}_{f}\right)
$$


are incoherent hermitian $\left(\boldsymbol{k}_{\mathbb{R}}, \widehat{\mathcal{O}}_{\boldsymbol{k}}\right)$-modules. Our small CM cycle is related to the cycle of [BHY15, $§ 5.1]$ by

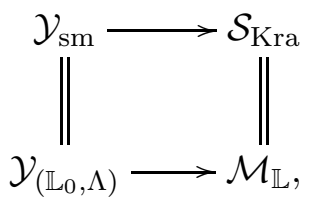

and the metrized line bundle $\widehat{\boldsymbol{\omega}}^{-1}$ of BHKRYa agrees with the metrized cotautological bundle $\hat{\mathcal{T}}_{\mathbb{L}}$ of BHY15].

Let $\Delta$ be the automorphism group of the finite abelian group $L^{\prime} / L$ endowed with the quadratic form $L^{\prime} / L \rightarrow \mathbb{Q} / \mathbb{Z}$ obtained by reduction of $Q: L \rightarrow \mathbb{Z}$. The tautological action of $\Delta$ on $S_{L}=\mathbb{C}\left[L^{\prime} / L\right]$ commutes with the Weil representation $\omega_{L}$, and hence $\Delta$ acts on all spaces of modular forms valued in the representation $\omega_{L}$.

Let $H_{2-n}\left(\omega_{L}\right)$ be the space of harmonic Maass forms of [BHY15, §2.2]. Every $f \in H_{2-n}\left(\omega_{L}\right)$ has a holomorphic part

$$
f^{+}(\tau)=\sum_{\substack{m \in \mathbb{Q} \\ m \gg-\infty}} c_{f}^{+}(m) \cdot q^{m},
$$

which is a formal $q$-expansion with coefficients in $S_{L}$. Let $c_{f}^{+}(0,0)$ be the value of $c_{f}^{+}(0) \in S_{L}$ at the trivial coset.

As in [BF04] or [BY09, §3.1], there is a $\Delta$-equivariant, surjective, conjugate linear differential operator

$$
\xi: H_{2-n}\left(\omega_{L}\right) \rightarrow S_{n}\left(\bar{\omega}_{L}\right),
$$

and the construction of [BHY15, (4.15)] defines a linear functional

$$
\widehat{\mathcal{Z}}: H_{2-n}\left(\omega_{L}\right)^{\Delta} \rightarrow \widehat{\mathrm{Ch}}_{\mathbb{C}}^{1}\left(\mathcal{S}_{\mathrm{Kra}}^{*}\right)
$$

These are related by the main result of [BHY15], which we now state.

Theorem 2.4.1 ([BHY15]). The equality

$$
\left[\widehat{\mathcal{Z}}(f): \mathcal{Y}_{\mathrm{sm}}\right]-c_{f}^{+}(0,0) \cdot\left[\widehat{\boldsymbol{\omega}}: \mathcal{Y}_{\mathrm{sm}}\right]=-\operatorname{deg}_{\mathbb{C}}\left(\mathcal{Y}_{\mathrm{sm}}\right) \cdot L^{\prime}\left(\xi(f), \theta_{\Lambda}, 0\right)
$$

holds for any $\Delta$-invariant $f \in H_{2-n}\left(\omega_{L}\right)$.

2.5. The proof of Theorem $\mathbf{A}$. Throughout 2.5 we assume $n \geqslant 3$. Under this assumption the linear functional (2.4.1) is closely related to the coefficients of the generating series (1.1.2). Indeed, If $m$ is a positive integer, [BHY15, Lemma 3.10] shows that there is a unique

$$
f_{m} \in H_{2-n}\left(\omega_{L}\right)^{\Delta}
$$

with holomorphic part

$$
f_{m}^{+}(\tau)=\phi_{0} \cdot q^{-m}+O(1),
$$


where $\phi_{0} \in S_{L}$ is the characteristic function of the trivial coset. Applying the above linear functional to this form recovers the $m^{\text {th }}$ coefficient

$$
\hat{\mathcal{Z}}_{\text {Kra }}^{\text {tot }}(m)=\hat{\mathcal{Z}}\left(f_{m}\right)
$$

of the generating series (1.1.2).

The following proposition explains the connection between the linear functional (2.4.1) and the arithmetic theta lift (1.1.3).

Proposition 2.5.1. For every $g \in S_{n}\left(\Gamma_{0}(D), \chi_{\boldsymbol{k}}^{n}\right)$ there is a $\Delta$-invariant form $f \in H_{2-n}\left(\omega_{L}\right)$ such that

$$
\hat{\theta}(g)=\hat{\mathcal{Z}}(f)+c_{f}^{+}(0,0) \cdot \hat{\mathcal{Z}}_{\mathrm{Kra}}^{\mathrm{tot}}(0),
$$

and such that $\xi(f)$ is equal to the form $\tilde{g} \in S_{n}\left(\bar{\omega}_{L}\right)$ defined by (2.3.2). Moreover, we may choose $f$ to be a linear combination of the forms $f_{m}$ characterized by (2.5.1).

Proof. Consider the space $H_{2-n}^{\infty}\left(\Gamma_{0}(D), \chi_{\boldsymbol{k}}^{n}\right)$ of harmonic Maass forms of BHKRYa, $\S 7.2$ ]. The constructions of BF04 provide us with a surjective conjugate linear differential operator

$$
\xi: H_{2-n}^{\infty}\left(\Gamma_{0}(D), \chi_{\boldsymbol{k}}^{n}\right) \rightarrow S_{n}\left(\Gamma_{0}(D), \chi_{\boldsymbol{k}}^{n}\right),
$$

and we choose an $f_{0} \in H_{2-n}^{\infty}\left(\Gamma_{0}(D), \chi_{\boldsymbol{k}}^{n}\right)$ such that $\xi\left(f_{0}\right)=g$. It is easily seen that $f_{0}$ may be chosen to vanish at all cusps of $\Gamma_{0}(D)$ different from $\infty$. This can, for instance, be attained by adding a suitable weakly holomorphic form in the space $M_{2-n}^{!, \infty}\left(\Gamma_{0}(D), \chi_{\boldsymbol{k}}^{n}\right)$ of [BHKRYa, $\left.\S 4.2\right]$. The Fourier expansion of the holomorphic part of $f_{0}$ is denoted

$$
f_{0}^{+}(\tau)=\sum_{m \in \mathbb{Q}} c_{0}^{+}(m) q^{m}
$$

As in (2.3.2), the form $f_{0}$ determines an $S_{L}$-valued harmonic Maass form

$$
f(\tau)=\sum_{\gamma \in \Gamma_{0}(D) \backslash \mathrm{SL}_{2}(\mathbb{Z})}\left(\left.f_{0}\right|_{2-n} \gamma\right)(\tau) \cdot \omega_{L}\left(\gamma^{-1}\right) \phi_{0} \in H_{2-n}\left(\omega_{L}\right)^{\Delta} .
$$

As the $\xi$-operator is equivariant for the action of $\mathrm{SL}_{2}(\mathbb{Z})$, we have $\xi(f)=$ $\tilde{g}$. According to BHKRYa, Proposition 6.1.2], which holds analogously for harmonic Maass forms, the coefficients of the holomorphic part $f^{+}$satisfy

$$
c_{f}^{+}(m, \mu)= \begin{cases}c_{0}^{+}(m) & \text { if } \mu=0 \\ 0 & \text { otherwise }\end{cases}
$$

for all $m \leqslant 0$. This equality implies that

$$
f=\sum_{m>0} c_{0}^{+}(-m) f_{m}
$$

where $f_{m} \in H_{2-n}\left(\omega_{L}\right)^{\Delta}$ is the harmonic form characterized by (2.5.1). Indeed, the difference between the two forms is a harmonic form $h$ whose holomorphic part $\sum_{m \geqslant 0} c_{h}^{+}(m) q^{m}$ has no principal part. It follows from 
BF04, Theorem 3.6] that such a harmonic form is actually holomorphic, and therefore vanishes because the weight is negative.

The above decomposition of $f$ as a linear combination of the $f_{m}$ 's implies that

$$
\widehat{\mathcal{Z}}(f)=\sum_{m>0} c_{0}^{+}(-m) \cdot \widehat{\mathcal{Z}}_{\mathrm{Kra}}^{\mathrm{tot}}(m) \in \widehat{\mathrm{Ch}}_{\mathbb{C}}^{1}\left(\mathcal{S}_{\mathrm{Kra}}^{*}\right),
$$

and consequently

$$
\begin{aligned}
\hat{\theta}(g) & =\left\langle\widehat{\phi}, \xi\left(f_{0}\right)\right\rangle_{\text {Pet }} \\
& =\left\{f_{0}, \widehat{\phi}\right\} \\
& =\sum_{m \geqslant 0} c_{0}^{+}(-m) \cdot \widehat{\mathcal{Z}}_{\mathrm{Kra}}^{\mathrm{tot}}(m) \\
& =\widehat{\mathcal{Z}}(f)+c_{f}^{+}(0,0) \cdot \hat{\mathcal{Z}}_{\mathrm{Kra}}^{\mathrm{tot}}(0) .
\end{aligned}
$$

Here, in the second line, we have used the bilinear pairing

$$
\{\cdot, \cdot\}: H_{2-n}^{\infty}\left(\Gamma_{0}(D), \chi_{\boldsymbol{k}}^{n}\right) \times M_{n}\left(\Gamma_{0}(D), \chi_{\boldsymbol{k}}^{n}\right) \rightarrow \mathbb{C}
$$

analogous to $\mathrm{BF} 04$, Proposition 3.5], and the fact that $f_{0}$ vanishes at all cusps different from $\infty$.

Remark 2.5.2. It is incorrectly claimed in BHY15, §1.3] that (2.5.2) holds for every form $f$ with $\xi(f)=\tilde{g}$.

The following is stated in the introduction as Theorem $\mathrm{A}$.

Theorem 2.5.3. If $g \in S_{n}\left(\Gamma_{0}(D), \chi_{\boldsymbol{k}}^{n}\right)$ and $\tilde{g} \in S_{n}\left(\bar{\omega}_{L}\right)$ are related by (2.3.2), then

$$
\left[\widehat{\theta}(g): \mathcal{Y}_{\mathrm{sm}}\right]=-\operatorname{deg}_{\mathbb{C}}\left(\mathcal{Y}_{\mathrm{sm}}\right) \cdot L^{\prime}\left(\tilde{g}, \theta_{\Lambda}, 0\right) .
$$

Proof. Choosing $f$ as in Proposition 2.5.1 and using the first equality of Proposition 2.2.10, yields

$$
\left[\hat{\theta}(g): \mathcal{Y}_{\mathrm{sm}}\right]=\left[\widehat{\mathcal{Z}}(f): \mathcal{Y}_{\mathrm{sm}}\right]-c_{f}^{+}(0,0) \cdot\left[\widehat{\boldsymbol{\omega}}: \mathcal{Y}_{\mathrm{sm}}\right]
$$

Thus the claim follows from Theorem 2.4.1,

\section{Further Results on the CONVOlution $L$-FunCtion}

In this section we specialize to the case where $g \in S_{n}\left(\Gamma_{0}(D), \chi_{\boldsymbol{k}}^{n}\right)$ is a new eigenform, and express the convolution $L$-function (2.3.3) associated to the vector valued cusp form $\tilde{g}$ in terms of the usual $L$-function associated to $g$.

This allows us, in Theorem 3.4 .1 below, to rewrite Theorem $\mathrm{A}$ of the introduction in a way that avoids vector-valued modular forms. When $n$ is even, it also allows us to formulate a version of Theorem $\mathrm{A}$ in which the $L$-function has an Euler product.

We assume $n \geqslant 2$ until we reach $\oint 3.4$, at which point we restrict to $n \geqslant 3$. 
3.1. Atkin-Lehner operators. Recall that $\chi_{\boldsymbol{k}}$ is the idele class character associated to the quadratic field $\boldsymbol{k}$. If we view $\chi_{\boldsymbol{k}}$ as a Dirichlet character modulo $D$, then any factorization $D=Q_{1} Q_{2}$ induces a factorization

$$
\chi_{\boldsymbol{k}}=\chi_{Q_{1}} \chi_{Q_{2}}
$$

where $\chi_{Q_{i}}:\left(\mathbb{Z} / Q_{i} \mathbb{Z}\right)^{\times} \rightarrow \mathbb{C}^{\times}$is a quadratic Dirichlet character.

Fix a normalized cuspidal new eigenform

$$
g(\tau)=\sum_{m>0} c(m) q^{m} \in S_{n}\left(\Gamma_{0}(D), \chi_{\boldsymbol{k}}^{n}\right) .
$$

As in [BHKRYa, Section 4.1], for each positive divisor $Q \mid D$, fix a matrix

$$
R_{Q}=\left(\begin{array}{cc}
\alpha & \beta \\
\frac{D}{Q} \gamma & Q \delta
\end{array}\right) \in \Gamma_{0}(D / Q)
$$

with $\alpha, \beta, \gamma, \delta \in \mathbb{Z}$, and define the Atkin-Lehner operator

$$
W_{Q}=\left(\begin{array}{cc}
Q \alpha & \beta \\
D \gamma & Q \delta
\end{array}\right)=R_{Q}\left(\begin{array}{ll}
Q & \\
& 1
\end{array}\right) .
$$

The cusp form

$$
\begin{aligned}
g_{Q}(\tau) & =\left.\chi_{Q}^{n}(\beta) \chi_{D / Q}^{n}(\alpha) \cdot g\right|_{n} W_{Q} \\
& =\sum_{m>0} c_{Q}(m) q^{m},
\end{aligned}
$$

is then independent of the choice of $\alpha, \beta, \gamma, \delta$.

Let $\epsilon_{Q}(g)$ be the fourth root of unity

$$
\epsilon_{Q}(g)=\prod_{\substack{q \mid Q \\ q \text { prime }}} \chi_{Q}^{n}(Q / q) \cdot \lambda_{q}
$$

where

$$
\lambda_{q}=\overline{c(q)} \cdot\left\{\begin{array}{lll}
-q^{1-\frac{n}{2}} & \text { if } n \equiv 0 \quad(\bmod 2) \\
\delta_{q} q^{\frac{1-n}{2}} & \text { if } n \equiv 1 \quad(\bmod 2),
\end{array}\right.
$$

and $\delta_{q}$ is defined by

$$
\delta_{q}=\left\{\begin{array}{lll}
1 & \text { if } q \equiv 1 & (\bmod 4) \\
i & \text { if } q \equiv 3 & (\bmod 4)
\end{array}\right.
$$

According to [Asa76, Theorem 2], we have

$$
\begin{aligned}
c_{Q}(m) & =\epsilon_{Q}(g) \chi_{Q}^{n}(m) c(m) & & \text { if }(m, Q)=1, \\
c_{Q}(m) & =\epsilon_{Q}(g) \chi_{D / Q}^{n}(m) \overline{c(m)} & & \text { if }(m, D / Q)=1, \\
c_{Q}\left(m_{1} m_{2}\right) & =\epsilon_{Q}(g)^{-1} c_{Q}\left(m_{1}\right) c_{Q}\left(m_{2}\right) & & \text { if }\left(m_{1}, m_{2}\right)=1 .
\end{aligned}
$$

Remark 3.1.1. If $n$ is even, then the Fourier coefficients of $g$ are totally real. It follows that $g_{Q}=\epsilon_{Q}(g) g$ for every divisor $Q \mid D$. Furthermore,

$$
\epsilon_{Q}(g)=\prod_{q \mid Q}\left(-q^{1-\frac{n}{2}} c(q)\right)= \pm 1 .
$$


3.2. Twisting theta functions. Let $\left(\mathfrak{a}_{0}, \mathfrak{a}_{1}, \mathfrak{b}\right)$ be a triple of self-dual hermitian $\mathcal{O}_{\boldsymbol{k}}$-lattices of signatures $(1,0),(0,1)$, and $(n-1,0)$, as in $\$ 2.2$, and recall that from this data we constructed hermitian $\mathcal{O}_{\boldsymbol{k}}$-lattices

$$
\mathfrak{a}=\mathfrak{a}_{1} \oplus \mathfrak{b}, \quad L=\operatorname{Hom}_{\mathcal{O}_{\boldsymbol{k}}}\left(\mathfrak{a}_{0}, \mathfrak{a}\right)
$$

of signature $(n-1,1)$. We also define

$$
L_{1}=\operatorname{Hom}_{\mathcal{O}_{\boldsymbol{k}}}\left(\mathfrak{a}_{0}, \mathfrak{a}_{1}\right), \quad \Lambda=\operatorname{Hom}_{\mathcal{O}_{\boldsymbol{k}}}\left(\mathfrak{a}_{0}, \mathfrak{b}\right),
$$

so that $L=L_{1} \oplus \Lambda$.

Let $\mathrm{GU}(\Lambda)$ be the unitary similitude group associated with $\Lambda$, viewed as an algebraic group over $\mathbb{Z}$. For any $\mathbb{Z}$-algebra $R$ its $R$-valued points are given by

$$
\mathrm{GU}(\Lambda)(R)=\left\{h \in \mathrm{GL}_{\mathcal{O}_{k}}\left(\Lambda_{R}\right):\langle h x, h y\rangle=\nu(h)\langle x, y\rangle \forall x, y \in \Lambda_{R}\right\},
$$

where $\nu(h) \in R^{\times}$denotes the similitude factor of $h$. Note the relation

$$
\mathrm{Nm}_{\boldsymbol{k} / \mathbb{Q}}(\operatorname{det}(h))=\nu(h)^{n-1} .
$$

For $h \in \mathrm{GU}(\Lambda)(\mathbb{R})$ the similitude factor $\nu(h)$ belongs to $\mathbb{R}_{>0}$.

As $\Lambda$ is positive definite, the set

$$
X_{\Lambda}=\mathrm{GU}(\Lambda)(\mathbb{Q}) \backslash \mathrm{GU}(\Lambda)\left(\mathbb{A}_{f}\right) / \mathrm{GU}(\Lambda)(\widehat{\mathbb{Z}})
$$

is finite. Denoting by

$$
\mathrm{CL}(\boldsymbol{k})=\boldsymbol{k}^{\times} \backslash \hat{\boldsymbol{k}}^{\times} / \widehat{\mathcal{O}}_{\boldsymbol{k}}^{\times}
$$

the ideal class group of $\boldsymbol{k}$, the natural map $\operatorname{Res}_{\boldsymbol{k} / \mathbb{Q}} \mathbb{G}_{m} \rightarrow \mathrm{GU}(\Lambda)$ to the center induces an action

$$
\mathrm{CL}(\boldsymbol{k}) \times X_{\Lambda} \longrightarrow X_{\Lambda}
$$

As in the proof of [BHKRYa, Proposition 2.1.1], any $h \in \operatorname{GU}(\Lambda)\left(\mathbb{A}_{f}\right)$ determines an $\mathcal{O}_{\boldsymbol{k}}$-lattice

$$
\Lambda_{h}=\Lambda_{\mathbb{Q}} \cap h \widehat{\Lambda} .
$$

This lattice is not self-dual under the hermitian form $\langle-,-\rangle$ on $\Lambda_{\mathbb{Q}}$. However, there is a unique positive rational number $\operatorname{rat}(\nu(h))$ such that

$$
\frac{\nu(h)}{\operatorname{rat}(\nu(h))} \in \widehat{\mathbb{Z}}^{\times},
$$

and the lattice $\Lambda_{h}$ is self-dual under the rescaled hermitian form

$$
\langle x, y\rangle_{h}=\frac{1}{\operatorname{rat}(\nu(h))} \cdot\langle x, y\rangle \text {. }
$$

If $h \in \mathrm{GU}(\Lambda)(\widehat{\mathbb{Z}})$ then $\Lambda_{h}=\Lambda$. If $h \in \mathrm{GU}(\Lambda)(\mathbb{Q})$, then $\Lambda_{h} \cong \Lambda$ as hermitian $\mathcal{O}_{\boldsymbol{k}}$-modules. Hence $h \mapsto \Lambda_{h}$ defines a function from $X_{\Lambda}$ to the set of isometry classes of self-dual hermitian $\mathcal{O}_{\boldsymbol{k}}$-module of signature $(n-1,0)$.

Similarly, for any $h \in \mathrm{GU}(\Lambda)\left(\mathbb{A}_{f}\right)$ we define a self-dual hermitian $\mathcal{O}_{\boldsymbol{k}^{-}}$ lattice of signature $(0,1)$ by endowing

$$
L_{1, h}=L_{1 \mathbb{Q}} \cap \operatorname{det}(h) \widehat{L}_{1}
$$


with the hermitian form

$$
\langle x, y\rangle_{h}=\frac{1}{\operatorname{rat}(\nu(h))^{n-1}} \cdot\langle x, y\rangle .
$$

The assignment $h \mapsto L_{1, h}$ defines a map from $X_{\Lambda}$ to the set of isometry classes of self-dual hermitian $\mathcal{O}_{\boldsymbol{k}}$-lattices of signature $(0,1)$.

Lemma 3.2.1. For any $h \in \mathrm{GU}(\Lambda)\left(\mathbb{A}_{f}\right)$ the hermitian $\mathcal{O}_{\boldsymbol{k}}$-lattice

$$
L_{h}=L_{1, h} \oplus \Lambda_{h}
$$

is isomorphic everywhere locally to $L$. Moreover, $L_{h}$ and $L$ become isomorphism after tensoring with $\mathbb{Q}$.

Proof. Let $p$ be a prime. As in [BHKRYa, §1.8], a $\boldsymbol{k}_{p}$-hermitian space is determined by its dimension and invariant. The relations

$$
\begin{aligned}
\operatorname{det}\left(\Lambda_{h} \otimes_{\mathbb{Z}} \mathbb{Q}\right) & =\operatorname{rat}(\nu(h))^{1-n} \cdot \operatorname{det}\left(\Lambda \otimes_{\mathbb{Z}} \mathbb{Q}\right), \\
\operatorname{det}\left(L_{1, h} \otimes_{\mathbb{Z}} \mathbb{Q}\right) & =\operatorname{rat}(\nu(h))^{1-n} \cdot \operatorname{det}\left(L_{1} \otimes_{\mathbb{Z}} \mathbb{Q}\right),
\end{aligned}
$$

combined with (3.2.3), imply that $L \otimes_{\mathbb{Z}} \mathbb{Q}$ and $L_{h} \otimes_{\mathbb{Z}} \mathbb{Q}$ have the same invariant everywhere locally. As they both have signature $(n-1,1)$, they are isomorphic everywhere locally, and hence isomorphic globally.

A result of Jacobowitz Jac62 shows that any two self-dual lattices in $L \otimes_{\mathbb{Z}} \mathbb{Q}$ are isomorphic everywhere locally, and hence it follows from the previous paragraph that $L$ and $L_{h}$ are isomorphic everywhere locally.

Define a linear map

$$
M_{n-1}\left(\omega_{\Lambda}^{\vee}\right) \rightarrow M_{n-1}\left(\Gamma_{0}(D), \chi_{\boldsymbol{k}}^{n-1}\right)
$$

from $S_{\Lambda}^{\vee}$-valued modular forms to scalar-valued modular forms by evaluation at the characteristic function $\phi_{0} \in S_{\Lambda}$ of the trivial coset $0 \in \Lambda^{\prime} / \Lambda$. This map takes the vector valued theta series $\theta_{\Lambda} \in M_{n-1}\left(\omega_{\Lambda}^{\vee}\right)$ of $₫ 2.3$ to the scalar valued theta series

$$
\theta_{\Lambda}^{\mathrm{sc}}(\tau)=\sum_{m \in \mathbb{Z}_{\geqslant 0}} R_{\Lambda}^{\mathrm{sc}}(m) \cdot q^{m}
$$

where $R_{\Lambda}^{\mathrm{sc}}(m)$ is the number of ways to represent $m$ by $\Lambda$.

Let $\eta$ be an algebraic automorphic form for $\mathrm{GU}(\Lambda)$ which is trivial at $\infty$ and right $\mathrm{GU}_{\Lambda}(\widehat{\mathbb{Z}})$-invariant. In other words, a function

$$
\eta: X_{\Lambda} \longrightarrow \mathbb{C} \text {. }
$$

Throughout we assume that under the action (3.2.4) the function $\eta$ transforms with a character $\chi_{\eta}: \mathrm{CL}(\boldsymbol{k}) \rightarrow \mathbb{C}^{\times}$, that is,

$$
\eta(\alpha h)=\chi_{\eta}(\alpha) \eta(h)
$$

We associate a theta function to $\eta$ by setting

$$
\theta_{\eta, \Lambda}^{\mathrm{sc}}=\sum_{h \in X_{\Lambda}} \frac{\eta(h)}{\left|\operatorname{Aut}\left(\Lambda_{h}\right)\right|} \cdot \theta_{\Lambda_{h}}^{\mathrm{sc}} \in M_{n-1}\left(\Gamma_{0}(D), \chi_{\boldsymbol{k}}^{n-1}\right) .
$$


This form is cuspidal when the character $\chi_{\eta}$ is non-trivial. We denote its Fourier expansion by

$$
\theta_{\eta, \Lambda}^{\mathrm{sc}}(\tau)=\sum_{m \geqslant 0} R_{\eta, \Lambda}^{\mathrm{sc}}(m) \cdot q^{m}
$$

Similarly, we may define

$$
\theta_{\eta, \Lambda}(\tau)=\sum_{h \in X_{\Lambda}} \frac{\eta(h)}{\left|\operatorname{Aut}\left(\Lambda_{h}\right)\right|} \cdot \theta_{\Lambda_{h}}(\tau),
$$

but this is only a formal sum: as $h$ varies the forms $\theta_{\Lambda_{h}}$ take values in the varying spaces $S_{\Lambda_{h}}^{\vee}$.

Lemma 3.2.1 allows us to identify $S_{L} \cong S_{L_{h}}$, and hence make sense of the $L$-function $L\left(\tilde{g}, \theta_{\Lambda_{h}}, s\right)$ as in (2.3.3). In the next subsection we will compare

$$
L\left(\tilde{g}, \theta_{\eta, \Lambda}, s\right)=\sum_{h \in X_{\Lambda}} \frac{\eta(h)}{\left|\operatorname{Aut}\left(\Lambda_{h}\right)\right|} \cdot L\left(\tilde{g}, \theta_{\Lambda_{h}}, s\right) .
$$

to the usual convolution $L$-function

$$
L\left(g, \theta_{\eta, \Lambda}^{\mathrm{sc}}, s\right)=\Gamma\left(\frac{s}{2}+n-1\right) \sum_{m=1}^{\infty} \frac{\overline{c(m)} R_{\eta, \Lambda}^{\mathrm{sc}}(m)}{(4 \pi m)^{\frac{s}{2}+n-1}}
$$

of the scalar-valued forms $g$ and $\theta_{\eta, \Lambda}^{\text {sc }}$.

3.3. Rankin-Selberg $L$-functions for scalar and vector valued forms. In this subsection we prove a precise relation between (3.2.6) and (3.2.7). First, we give an explicit formula for the Fourier coefficients $a(m, \mu)$ of $\tilde{g}$ in terms of those of $g$ analogous to BHKRYa, Proposition 6.1.2].

For a prime $p$ dividing $D$ define

$$
\gamma_{p}=\delta_{p}^{-n} \cdot(D, p)_{p}^{n} \cdot \operatorname{inv}_{p}\left(V_{p}\right) \in\{ \pm 1, \pm i\},
$$

where $\operatorname{inv}_{p}\left(V_{p}\right)$ is the invariant of $V_{p}=\operatorname{Hom}_{\boldsymbol{k}}\left(W_{0}, W\right) \otimes_{\mathbb{Q}} \mathbb{Q}_{p}$ in the sense of BHKRYa, (1.8.3)] and $\delta_{p} \in\{1, i\}$ is as before. It is equal to the local Weil index of the Weil representation of $\mathrm{SL}_{2}\left(\mathbb{Z}_{p}\right)$ on $S_{L_{p}} \subset S\left(V_{p}\right)$, where $V_{p}$ is viewed as a quadratic space by taking the trace of the hermitian form. This is explained in more detail in BHKRYa, Section 8.1]. For any $Q$ dividing $D$ we define

$$
\gamma_{Q}=\prod_{q \mid Q} \gamma_{q} .
$$

Remark 3.3.1. If $n$ is even and $p \mid D$, then (3.3.1) simplifies to

$$
\gamma_{p}=\left(\frac{-1}{p}\right)^{n / 2} \operatorname{inv}_{p}\left(V_{p}\right) .
$$

For any $\mu \in L^{\prime} / L$ define $Q_{\mu} \mid D$ by

$$
Q_{\mu}=\prod_{\substack{p \mid D \\ \mu_{p} \neq 0}} p
$$


where $\mu_{p}$ is the image of $\mu$ in $L_{p}^{\prime} / L_{p}$. Let $\phi_{\mu} \in S_{L}$ be the characteristic function of $\mu$.

Proposition 3.3.2. For all $m \in \mathbb{Q}$ the coefficients $\tilde{a}(m) \in S_{L}$ of $\tilde{g}$ satisfy

$$
\tilde{a}(m, \mu)= \begin{cases}\sum_{Q_{\mu}|Q| D} Q^{1-n} \overline{\gamma_{Q}} \cdot c_{Q}(m Q) & \text { if } m \equiv-Q(\mu) \quad(\bmod \mathbb{Z}), \\ 0 & \text { otherwise. }\end{cases}
$$

Proof. The first formula is a special case of results of Scheithauer Sch09, Section 5]. It can also be proved in the same way as Proposition 6.1 .2 of BHKRYa. The complex conjugation over $\gamma_{Q}$ arises because of the fact that $\tilde{g}$ transforms with the complex conjugate representation $\bar{\omega}_{L}$. The additional factor $Q^{1-n}$ is due to the fact that we work here in weight $n$.

Proposition 3.3.3. The convolution L-function (2.3.3) satisfies

$$
L\left(\tilde{g}, \theta_{\Lambda}, s\right)=\sum_{Q \mid D} Q^{\frac{s}{2}} \gamma_{Q} \cdot L\left(g_{Q}, \theta_{\Lambda_{\mathfrak{q}}}^{\mathrm{sc}}, s\right)
$$

where $\mathfrak{q} \in \widehat{\boldsymbol{k}}^{\times}$is such that $\mathfrak{q}^{2} \widehat{\mathcal{O}}_{\boldsymbol{k}}^{\times}=Q \widehat{\mathcal{O}}_{\boldsymbol{k}}^{\times}$. Moreover, for any $\eta: X_{\Lambda} \rightarrow \mathbb{C}$ satisfying (3.2.5) the $L$-functions (3.2.6) and (3.2.7) are related by

$$
L\left(\tilde{g}, \theta_{\eta, \Lambda}, s\right)=\sum_{Q \mid D} Q^{\frac{s}{2}} \gamma_{Q} \cdot \chi_{\eta}\left(\mathfrak{q}^{-1}\right) L\left(g_{Q}, \theta_{\eta, \Lambda}^{\mathrm{sc}}, s\right) .
$$

Proof. Proposition 3.3.2 implies

$$
\begin{aligned}
\frac{L\left(\tilde{g}, \theta_{\Lambda}, s\right)}{\Gamma\left(\frac{s}{2}+n-1\right)} & =\sum_{\mu \in \Lambda^{\prime} / \Lambda} \sum_{m \in \mathbb{Q}_{>0}} \sum_{Q_{\mu}|Q| D} Q^{1-n} \gamma_{Q} \cdot \frac{\overline{c_{Q}(m Q)} R_{\Lambda}\left(m, \phi_{\mu}\right)}{(4 \pi m)^{\frac{s}{2}+n-1}} \\
& =\sum_{Q \mid D} Q^{1-n} \gamma_{Q} \sum_{m \in \frac{1}{Q} \mathbb{Z}_{>0}} \frac{\overline{c_{Q}(m Q)}}{(4 \pi m)^{\frac{s}{2}+n-1}} \sum_{\substack{\mu \in \Lambda^{\prime} / \Lambda \\
Q_{\mu} \mid Q}} R_{\Lambda}\left(m, \phi_{\mu}\right) \\
& =\sum_{Q \mid D} Q^{\frac{s}{2}} \gamma_{Q} \sum_{m \in \mathbb{Z}_{>0}} \frac{\overline{c_{Q}(m)}}{(4 \pi m)^{\frac{s}{2}+n-1}} \sum_{\substack{\mu \in \Lambda^{\prime} / \Lambda \\
Q_{\mu} \mid Q}} R_{\Lambda}\left(m / Q, \phi_{\mu}\right) .
\end{aligned}
$$

The first claim now follows from the relation

$$
\sum_{\substack{\mu \in \Lambda^{\prime} / \Lambda \\ Q_{\mu} \mid Q}} R_{\Lambda}(m / Q, \mu)=R_{\Lambda_{\mathfrak{q}^{-1}}}(m, 0)=R_{\Lambda_{\mathfrak{q}}}(m, 0) .
$$

For the second claim, if we replace $\Lambda$ by $\Lambda_{h}$ and $L_{1}$ by $L_{1, h}$ for $h \in X_{\Lambda}$, then $L$ and $\gamma_{Q}$ remain unchanged. The above calculations therefore imply 
that

$$
\begin{aligned}
L\left(\tilde{g}, \theta_{\eta, \Lambda}, s\right) & =\sum_{Q \mid D} \gamma_{Q} Q^{\frac{s}{2}} \sum_{h \in X_{\Lambda}} \frac{\eta(h)}{\left|\operatorname{Aut}\left(\Lambda_{h}\right)\right|} L\left(g_{Q}, \theta_{\Lambda_{\mathfrak{q} h}}^{\mathrm{sc}}, s\right) \\
& =\sum_{Q \mid D} \gamma_{Q} Q^{\frac{s}{2}} \sum_{h \in X_{\Lambda}} \frac{\eta\left(\mathfrak{q}^{-1} h\right)}{\left|\operatorname{Aut}\left(\Lambda_{h}\right)\right|} L\left(g_{Q}, \theta_{\Lambda_{h}}^{\mathrm{sc}}, s\right) \\
& =\sum_{Q \mid D} \gamma_{Q} Q^{\frac{s}{2}} \cdot \chi_{\eta}\left(\mathfrak{q}^{-1}\right) L\left(g_{Q}, \theta_{\eta, \Lambda}^{\mathrm{sc}}, s\right),
\end{aligned}
$$

where we have used (3.2.5) and the fact that $\left|\operatorname{Aut}\left(\Lambda_{h}\right)\right|=\left|\operatorname{Aut}\left(\Lambda_{\mathfrak{q} h}\right)\right|$.

Corollary 3.3.4. If $n$ is even, then

$$
L\left(\tilde{g}, \theta_{\eta, \Lambda}, s\right)=L\left(g, \theta_{\eta, \Lambda}^{\mathrm{sc}}, s\right) \cdot \prod_{p \mid D}\left(1+\chi_{\eta}\left(\mathfrak{p}^{-1}\right) \epsilon_{p}(g) \gamma_{p} p^{\frac{s}{2}}\right) .
$$

Proof. This is immediate from Proposition 3.3 .3 and Remark 3.1.1.

3.4. Small CM cycles and derivatives of $L$-functions, revisited. Now we are ready to state a variant of Theorem A using only scalar valued modular forms. Assume $n \geqslant 3$.

Every $h \in X_{\Lambda}$ determines a codimension $n-1$ cycle

$$
\mathcal{Y}_{\mathrm{sm}, h} \rightarrow \mathcal{S}_{\mathrm{Kra}}^{*}
$$

as follows. From the triple $\left(\mathfrak{a}_{0}, \mathfrak{a}_{1}, \mathfrak{b}\right)$ fixed in $\$ 3.2$ and the hermitian $\mathcal{O}_{\boldsymbol{k}^{-}}$ lattices $L_{h}=L_{1, h} \oplus \Lambda_{h}$ of Lemma 3.2.1, we denote by $\mathfrak{a}_{1, h}$ and $\mathfrak{b}_{h}$ the unique hermitian $\mathcal{O}_{k}$-lattices satisfying

$$
L_{1, h} \cong \operatorname{Hom}_{\mathcal{O}_{\boldsymbol{k}}}\left(\mathfrak{a}_{0}, \mathfrak{a}_{1, h}\right), \quad \Lambda_{h} \cong \operatorname{Hom}_{\mathcal{O}_{\boldsymbol{k}}}\left(\mathfrak{a}_{0}, \mathfrak{b}_{h}\right),
$$

and set $\mathfrak{a}_{h}=\mathfrak{a}_{1, h} \oplus \mathfrak{b}_{h}$ so that $L_{h} \cong \operatorname{Hom}_{\mathcal{O}_{\boldsymbol{k}}}\left(\mathfrak{a}_{0}, \mathfrak{a}_{h}\right)$. Compare with (3.2.1) and (3.2.2).

Repeating the construction of the small $\mathrm{CM}$ cycle $\mathcal{Y}_{\mathrm{sm}}$ with the triple $\left(\mathfrak{a}_{0}, \mathfrak{a}_{1}, \mathfrak{b}\right)$ replaced by $\left(\mathfrak{a}_{0}, \mathfrak{a}_{1, h}, \mathfrak{b}_{h}\right)$ results in a proper étale $\mathcal{O}_{\boldsymbol{k}}$-stack $\mathcal{Y}_{\mathrm{sm}, h}$. Repeating the construction of the Shimura variety $\mathcal{S}_{\text {Kra }}$ with the triple $\left(\mathfrak{a}_{0}, \mathfrak{a}\right)$ replaced by $\left(\mathfrak{a}_{0}, \mathfrak{a}_{h}\right)$ results in a new Shimura variety $\mathcal{S}_{\mathrm{Kra}, h}$, along with a finite and unramified morphism

$$
\mathcal{Y}_{\mathrm{sm}, h} \rightarrow \mathcal{S}_{\mathrm{Kra}, h} .
$$

It follows from Lemma 3.2 .1 that $\mathfrak{a}$ and $\mathfrak{a}_{h}$ are isomorphic everywhere locally, and examination of the moduli problem defining $\mathcal{S}_{\mathrm{Kra}}$ in BHKRYa, $\S 2.3$ ] shows that $\mathcal{S}_{\mathrm{Kra}}$ depends only the everywhere local data determined by the pair $\left(\mathfrak{a}_{0}, \mathfrak{a}\right)$, and not on the actual global $\mathcal{O}_{\boldsymbol{k}}$-hermitian lattices. Therefore, there is a canonical morphism of $\mathcal{O}_{\boldsymbol{k}}$-stacks

$$
\mathcal{Y}_{\mathrm{sm}, h} \rightarrow \mathcal{S}_{\mathrm{Kra}, h} \cong \mathcal{S}_{\mathrm{Kra}}
$$

in which the isomorphism is simply the identity functor on the moduli problems. In the end, this amounts to simply repeating the construction of 
$\mathcal{Y}_{\mathrm{sm}} \rightarrow \mathcal{S}_{\mathrm{Kra}}$ from Definition 2.2.6 word-for-word, but replacing $\Lambda$ by $\Lambda_{h}$ everywhere. This defines the desired cycle (3.4.1).

Each algebraic automorphic form $\eta: X_{\Lambda} \rightarrow \mathbb{C}$ satisfying (3.2.5) now determines a cycle

$$
\eta \mathcal{Y}_{\mathrm{sm}}=\sum_{h \in X_{\Lambda}} \eta(h) \cdot \mathcal{Y}_{\mathrm{sm}, h}
$$

on $\mathcal{S}_{\mathrm{Kra}}^{*}$ with complex coefficients, and a corresponding linear functional

$$
\left[-: \eta \mathcal{Y}_{\mathrm{sm}}\right]: \widehat{\mathrm{Ch}}_{\mathbb{C}}^{1}\left(\mathcal{S}_{\mathrm{Kra}}^{*}\right) \rightarrow \mathbb{C}
$$

Theorem 3.4.1. The arithmetic theta lift (1.1.3) satisfies

$$
\left[\hat{\theta}(g): \mathcal{Y}_{\mathrm{sm}}\right]=-\left.\operatorname{deg}_{\mathbb{C}}\left(\mathcal{Y}_{\mathrm{sm}}\right) \cdot \frac{d}{d s}\left[\sum_{Q \mid D} Q^{\frac{s}{2}} \gamma_{Q} L\left(g_{Q}, \theta_{\Lambda_{\mathfrak{q}}}^{\mathrm{sc}}, s\right)\right]\right|_{s=0}
$$

where $\mathfrak{q} \in \widehat{\boldsymbol{k}}^{\times}$is such that $\mathfrak{q}^{2} \widehat{\mathcal{O}}_{\boldsymbol{k}}^{\times}=Q \widehat{\mathcal{O}}_{\boldsymbol{k}}^{\times}$. Moreover, if $n$ is even and $\eta: X_{\Lambda} \rightarrow \mathbb{C}$ satisfies (3.2.5), then

$$
\begin{aligned}
& {\left[\hat{\theta}(g): \eta \mathcal{Y}_{\mathrm{sm}}\right]} \\
& =-\left.2^{1-o\left(d_{\boldsymbol{k}}\right)}\left(h_{\boldsymbol{k}} / w_{\boldsymbol{k}}\right)^{2} \cdot \frac{d}{d s}\left[L\left(g, \theta_{\eta, \Lambda}^{\mathrm{sc}}, s\right) \cdot \prod_{p \mid D}\left(1+\chi_{\eta}\left(\mathfrak{p}^{-1}\right) \epsilon_{p}(g) \gamma_{p} p^{\frac{s}{2}}\right)\right]\right|_{s=0},
\end{aligned}
$$

where $\mathfrak{p} \in \widehat{\boldsymbol{k}}^{\times}$such that $\mathfrak{p}^{2} \widehat{\mathcal{O}}_{\boldsymbol{k}}^{\times}=p \widehat{\mathcal{O}}_{\boldsymbol{k}}^{\times}$. Note that in the first formula the sum is over all positive divisors $Q \mid D$, while in the second the product is over the prime divisors $p \mid D$.

Proof. The first assertion follows from Theorem $\mathrm{A}$ and Proposition 3.3 .3 .

For the second assertion, applying Theorem $\mathrm{A}$ to

$$
\mathcal{Y}_{\mathrm{sm}, h} \rightarrow \mathcal{S}_{\mathrm{Kra}, h}^{*} \cong \mathcal{S}_{\mathrm{Kra}}^{*}
$$

yields

$$
\left[\hat{\theta}(g): \mathcal{Y}_{\mathrm{sm}, h}\right]=-\left.\operatorname{deg}_{\mathbb{C}}\left(\mathcal{Y}_{\mathrm{sm}, h}\right) \cdot \frac{d}{d s} L\left(\tilde{g}, \theta_{\Lambda_{h}}, s\right)\right|_{s=0} .
$$

Combining this with Proposition 2.2.9 yields

$$
\left[\widehat{\theta}(g): \eta \mathcal{Y}_{\mathrm{sm}}\right]=-\left.2^{1-o\left(d_{\boldsymbol{k}}\right)}\left(h_{\boldsymbol{k}} / w_{\boldsymbol{k}}\right)^{2} \cdot \frac{d}{d s} L\left(\tilde{g}, \theta_{\eta, \Lambda}, s\right)\right|_{s=0},
$$

and an application of Corollary 3.3.4 completes the proof.

Remark 3.4.2. Since the $L$-function $(3.2 .6)$ vanishes at $s=0$, the same must be true for the expressions in brackets on the right hand sides of the equalities of the above theorem. In particular, when $n$ is even, then either $L\left(g, \theta_{\eta, \Lambda}^{\mathrm{sc}}, s\right)$ or at least one of the factors

$$
1+\chi_{\eta}\left(\mathfrak{p}^{-1}\right) \epsilon_{p}(g) \gamma_{p} p^{\frac{s}{2}}
$$


(for a prime $p \mid D$ ) vanishes at $s=0$. If we pick the newform $g$ such that the latter local factors are nonvanishing, then $L\left(g, \theta_{\eta, \Lambda}^{\mathrm{sc}}, 0\right)=0$ and we obtain

$$
\left[\hat{\theta}(g): \eta \mathcal{Y}_{\mathrm{sm}}\right]=-2^{1-o\left(d_{\boldsymbol{k}}\right)} \frac{h_{\boldsymbol{k}}^{2}}{w_{\boldsymbol{k}}^{2}} \cdot \prod_{p \mid D}\left(1+\chi_{\eta}\left(\mathfrak{p}^{-1}\right) \epsilon_{p}(g) \gamma_{p}\right) \cdot L^{\prime}\left(g, \theta_{\eta, \Lambda}^{\mathrm{sc}}, 0\right) .
$$

\section{Big CM CYClES AND DERIVATIVES OF $L$-FUnCtions}

In this section we prove Theorem B by combining results of BHKRYa and [How12, How15, BKY12]. We asume $n \geqslant 2$ until 4.4 , at which point we restrict to $n \geqslant 3$.

4.1. A Shimura variety of dimension zero. Let $F$ be a totally real field of degree $n$, and define a CM field $E=\boldsymbol{k} \otimes_{\mathbb{Q}} F$. Define a rank $n+2$ torus $T_{\text {big }}$ over $\mathbb{Q}$ as the fiber product

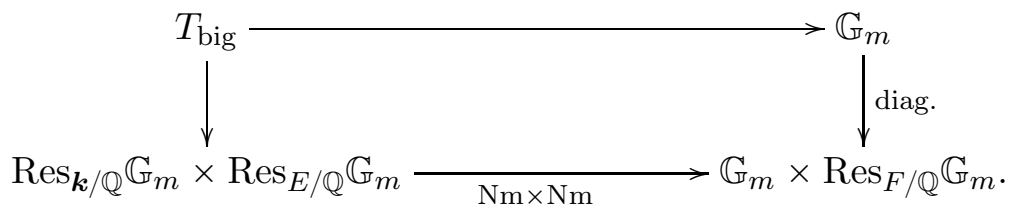

Its group of $\mathbb{Q}$-points is

$$
T_{\text {big }}(\mathbb{Q}) \cong\left\{(x, y) \in \boldsymbol{k}^{\times} \times E^{\times}: x \bar{x}=y \bar{y}\right\} .
$$

Remark 4.1.1. There is an isomorphism

$$
T_{\text {big }}(\mathbb{Q}) \cong \boldsymbol{k}^{\times} \times \operatorname{ker}\left(\mathrm{Nm}: E^{\times} \rightarrow F^{\times}\right)
$$

defined by $(x, y) \mapsto\left(x, x^{-1} y\right)$. It is clear that this arises from an isomorphism

$$
T_{\text {big }} \cong \operatorname{Res}_{\boldsymbol{k} / \mathbb{Q}} \mathbb{G}_{m} \times \operatorname{ker}\left(\mathrm{Nm}: \operatorname{Res}_{E / \mathbb{Q}} \mathbb{G}_{m} \rightarrow \operatorname{Res}_{F / \mathbb{Q}} \mathbb{G}_{m}\right) \text {. }
$$

As in the discussion preceding Theorem $\mathbb{B}$, let $\Phi \subset \operatorname{Hom}_{\mathbb{Q}}(E, \mathbb{C})$ be a CM type of signature $(n-1,1)$, let

$$
\varphi^{\mathrm{sp}}: E \rightarrow \mathbb{C}
$$

be its special element, and let $\mathcal{O}_{\Phi}$ be the ring of integers of $E_{\Phi}=\varphi^{\mathrm{sp}}(E)$.

The CM type $\Phi$ determines an isomorphism $\mathbb{C}^{n} \cong E_{\mathbb{R}}$, and hence an embedding $\mathbb{C}^{\times} \rightarrow E_{\mathbb{R}}^{\times}$arising from a morphism of real algebraic groups $\mathbb{S} \rightarrow\left(\operatorname{Res}_{E / \mathbb{Q}} \mathbb{G}_{m}\right)_{\mathbb{R}}$. This induces a morphism

$$
\mathbb{S} \rightarrow\left(\operatorname{Res}_{\boldsymbol{k} / \mathbb{Q}} \mathbb{G}_{m}\right)_{\mathbb{R}} \times\left(\operatorname{Res}_{E / \mathbb{Q}} \mathbb{G}_{m}\right)_{\mathbb{R}}
$$

which factors through a morphism

$$
h_{\text {big }}: \mathbb{S} \rightarrow T_{\text {big }, \mathbb{R}}
$$

The pair $\left(T_{\text {big }},\left\{h_{\text {big }}\right\}\right)$ is a Shimura datum, which, along with the compact open subgroup

$$
K_{\text {big }}=T_{\text {big }}\left(\mathbb{A}_{f}\right) \cap\left(\widehat{\mathcal{O}}_{\boldsymbol{k}}^{\times} \times \widehat{\mathcal{O}}_{E}^{\times}\right),
$$

determines a 0 -dimensional $E_{\Phi^{-}}$-stack $\operatorname{Sh}\left(T_{\text {big }}\right)$ with complex points

$$
\operatorname{Sh}\left(T_{\text {big }}\right)(\mathbb{C})=T_{\text {big }}(\mathbb{Q}) \backslash\left\{h_{\text {big }}\right\} \times T_{\text {big }}\left(\mathbb{A}_{f}\right) / K_{\text {big }} .
$$


4.2. The big CM cycle. The Shimura variety just constructed has a moduli interpretation, which we will use to construct an integral model. The interpretation we have in mind requires first choosing a triple $\left(\mathfrak{a}_{0}, \mathfrak{a}, i_{E}\right)$ in which

- $\mathfrak{a}_{0}$ is a self-dual hermitian $\mathcal{O}_{\boldsymbol{k}}$-lattice of signature $(1,0)$,

- $\mathfrak{a}$ is a self-dual hermitian $\mathcal{O}_{\boldsymbol{k}}$-lattice of signature $(n-1,1)$,

- $i_{E}: \mathcal{O}_{E} \rightarrow \operatorname{End}_{\mathcal{O}_{\boldsymbol{k}}}(\mathfrak{a})$ is an action extending the action of $\mathcal{O}_{\boldsymbol{k}}$.

Denoting by $H: \mathfrak{a} \times \mathfrak{a} \rightarrow \mathcal{O}_{\boldsymbol{k}}$ the hermitian form, we require further that

$$
H\left(i_{E}(x) a, b\right)=H\left(a, i_{E}(\bar{x}) b\right)
$$

for all $x \in \mathcal{O}_{E}$ and $a, b \in \mathfrak{a}$, and that in the decomposition

$$
\mathfrak{a}_{\mathbb{R}} \cong \bigoplus_{\varphi_{F}: F \rightarrow \mathbb{R}} \mathfrak{a} \otimes_{\mathcal{O}_{F}, \varphi_{F}} \mathbb{R}
$$

the summand indexed by $\varphi_{F}=\left.\varphi^{\mathrm{sp}}\right|_{F}$ is negative definite (which, by the signature condition, implies that the other summands are positive definite).

Remark 4.2.1. In general such a triple need not exist. In the applications will assume that the discriminants of $\boldsymbol{k} / \mathbb{Q}$ and $F / \mathbb{Q}$ are odd and relatively prime, and in this case one can construct such a triple using the argument of [How12, Proposition 3.1.6].

We now define a moduli space of abelian varieties with complex multiplication by $\mathcal{O}_{E}$ and type $\Phi$, as in [How12, $\left.\S 3.1\right]$. Denote by $\mathcal{C} \mathcal{M}_{\Phi}$ the functor that associates to every $\mathcal{O}_{\Phi}$-scheme $S$ the groupoid of triples $(A, \iota, \psi)$ in which

- $A \rightarrow S$ is an abelian scheme of dimenension $n$,

- $\iota: \mathcal{O}_{E} \rightarrow \operatorname{End}(A)$ is an $\mathcal{O}_{E}$-action,

- $\psi: A \rightarrow A^{\vee}$ is a principal polarization such that

$$
\iota(x)^{\vee} \circ \psi=\psi \circ \iota(\bar{x})
$$

for all $x \in \mathcal{O}_{E}$.

We also impose the $\Phi$-determinant condition that every $x \in \mathcal{O}_{E}$ acts on $\operatorname{Lie}(A)$ with characteristic polynomial equal to the image of

$$
\prod_{\varphi \in \Phi}(T-\varphi(x)) \in \mathcal{O}_{\Phi}[T]
$$

in $\mathcal{O}_{S}[T]$. We usually abbreviate $A \in \mathcal{C} \mathcal{M}_{\Phi}(S)$, and suppress the data $\iota$ and $\psi$ from the notation. By [How12, Proposition 3.1.2], the functor $\mathcal{C M}_{\Phi}$ is represented by a Deligne-Mumford stack, proper and étale over $\mathcal{O}_{\Phi}$.

Remark 4.2.2. The $\Phi$-determinant condition defined above agrees with that of [How12, §3.1]. As in Har15, Proposition 2.1.3], this is a consequence of Amitsur's formula, which can be found in Ami80, Theorem A] or [Che14, Lemma 1.12]. 
Define an open and closed substack

$$
\mathcal{Y}_{\text {big }} \subset \mathcal{M}_{(1,0)} \times \mathcal{O}_{k} \mathcal{C} \mathcal{M}_{\Phi}
$$

as the union of connected components $\mathcal{B} \subset \mathcal{M}_{(1,0)} \times \mathcal{O}_{k} \mathcal{C} \mathcal{M}_{\Phi}$ satisfying the following property: for every complex point $y=\left(A_{0}, A\right) \in \mathcal{B}(\mathbb{C})$, and for all primes $\ell$, there is an $\mathcal{O}_{E}$-linear isomorphism of hermitian $\mathcal{O}_{\boldsymbol{k}, \ell}$-lattices

$$
\operatorname{Hom}_{\mathcal{O}_{\boldsymbol{k}, \ell}}\left(A_{0}\left[\ell^{\infty}\right], A\left[\ell^{\infty}\right]\right) \cong \operatorname{Hom}_{\mathcal{O}_{\boldsymbol{k}}}\left(\mathfrak{a}_{0}, \mathfrak{a}\right) \otimes_{\mathbb{Z}} \mathbb{Q}_{\ell} \text {. }
$$

Remark 4.2.3. To verify that a connected component $\mathcal{B} \subset \mathcal{M}_{(1,0)} \times \mathcal{O}_{k} \mathcal{C M}_{\Phi}$ is contained in $\mathcal{Y}_{\text {big }}$, it suffices to check that (4.2.1) holds for one complex point $y \in \mathcal{B}(\mathbb{C})$. This is a consequence of the main theorem of complex multiplication and the fact that the points of $\mathcal{B}(\mathbb{C})$ form a single $\operatorname{Aut}\left(\mathbb{C} / E_{\Phi}\right)$ orbit.

Proposition 4.2.4. There is a canonical isomorphism of $E_{\Phi}$-stacks

$$
\operatorname{Sh}\left(T_{\text {big }}\right) \cong \mathcal{Y}_{\text {big } / E_{\Phi}} .
$$

Proof. The natural actions of $\mathcal{O}_{\boldsymbol{k}}$ and $\mathcal{O}_{E}$ on $\mathfrak{a}_{0}$ and $\mathfrak{a}$ determine an action of the subtorus

$$
T_{\text {big }} \subset \operatorname{Res}_{\boldsymbol{k} / \mathbb{Q}} \mathbb{G}_{m} \times \operatorname{Res}_{E / \mathbb{Q}} \mathbb{G}_{m}
$$

on $\mathfrak{a}_{0 \mathbb{Q}}$ and $\mathfrak{a}_{\mathbb{Q}}$, and the morphism $h_{\text {big }}: \mathbb{S} \rightarrow T_{\mathrm{sm}, \mathbb{R}}$ endows each of the real vector spaces $\mathfrak{a}_{0 \mathbb{R}}$ and $\mathfrak{a}_{\mathbb{R}}$ with a complex structure.

The desired isomorphism on complex points sends

$$
\left(h_{\mathrm{big}}, g\right) \in \operatorname{Sh}\left(T_{\mathrm{sm}}\right)(\mathbb{C})
$$

to the pair $\left(A_{0}, A\right)$ defined by

$$
A_{0}(\mathbb{C})=\mathfrak{a}_{0 \mathbb{R}} / g \mathfrak{a}_{0}, \quad A(\mathbb{C})=\mathfrak{a}_{\mathbb{R}} / g \mathfrak{a} .
$$

The elliptic curve $A_{0}$ is endowed with its natural $\mathcal{O}_{\boldsymbol{k}}$-action and its unique principal ploarization. The abelian variety $A$ is endowed with its natural $\mathcal{O}_{E}$-action, and the polarization induced by the symplectic form determined by its $\mathcal{O}_{\boldsymbol{k}}$-hermitian form, as in the proof of [BHKRYa, Proposition 2.2.1].

It follows from the theory of canonical models that this isomorphism on complex points descends to an isomorphism of $E_{\Phi^{-} \text {-stacks. }}$

The triple $\left(\mathfrak{a}_{0}, \mathfrak{a}, i_{E}\right)$ determines a pair $\left(\mathfrak{a}_{0}, \mathfrak{a}\right)$ as in the introduction, which determines a unitary Shimura variety with integral model $\mathcal{S}_{\mathrm{Kra}}$ as in (1.1.1). Recalling that $\mathcal{O}_{\boldsymbol{k}} \subset \mathcal{O}_{\Phi}$ as subrings of $\mathbb{C}$, we now view both $\mathcal{Y}_{\text {big }}$ and $\mathcal{C} \mathcal{M}_{\Phi}$ as $\mathcal{O}_{\boldsymbol{k}}$-stacks. There is a commutative diagram

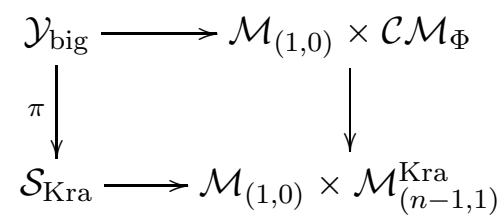

(all fiber products are over $\mathcal{O}_{\boldsymbol{k}}$ ), in which the vertical arrow on the right is the identity on the first factor and "forget complex multiplication" on the second. The arrow $\pi$ is defined by the commutativity of the diagram. 
Remark 4.2.5. In order to define the morphism

$$
\mathcal{C} \mathcal{M}_{\Phi} \rightarrow \mathcal{M}_{(n-1,1)}^{\mathrm{Kra}}
$$

in the diagram above, we must endow a point $A \in \mathcal{C M}_{\Phi}(S)$ with a subsheaf $\mathcal{F}_{A} \subset \operatorname{Lie}(A)$ satisfying Krämer's condition [BHKRYa, §2.3]. Using the morphism

$$
\mathcal{O}_{E} \stackrel{\varphi^{\mathrm{sp}}}{\longrightarrow} \mathcal{O}_{\Phi} \rightarrow \mathcal{O}_{S}
$$

denote by $J_{\varphi^{\text {sp }}} \subset \mathcal{O}_{E} \otimes_{\mathbb{Z}} \mathcal{O}_{S}$ the kernel of

$$
\mathcal{O}_{E} \otimes_{\mathbb{Z}} \mathcal{O}_{S} \stackrel{x \otimes y \mapsto \varphi^{\mathrm{sp}}(x) \cdot y}{\longrightarrow} \mathcal{O}_{S} .
$$

According to [How15, Lemma 4.1.2], the subsheaf $\mathcal{F}_{A}=J_{\varphi^{\mathrm{sp}}} \operatorname{Lie}(A)$ has the desired properties.

Definition 4.2.6. Composing the morphism $\pi$ in the diagram above with the inclusion of $\mathcal{S}_{\mathrm{Kra}}$ into its toroidal compactification, we obtain a morphism of $\mathcal{O}_{\boldsymbol{k}}$-stacks

called the big CM cycle.

$$
\pi: \mathcal{Y}_{\text {big }} \rightarrow \mathcal{S}_{\text {Kra }}^{*}
$$

Exactly as in $\$ 2.2$, the arithmetic degree along $\mathcal{Y}_{\text {big }}$ is the composition

$$
\widehat{\mathrm{Ch}}_{\mathbb{C}}^{1}\left(\mathcal{S}_{\mathrm{Kra}}^{*}\right) \stackrel{\pi^{*}}{\longrightarrow} \widehat{\mathrm{Ch}}_{\mathbb{C}}^{1}\left(\mathcal{Y}_{\mathrm{big}}\right) \stackrel{\widehat{\mathrm{deg}}}{\longrightarrow} \mathbb{C}
$$

We denote this linear functional by $\widehat{\mathcal{Z}} \mapsto\left[\widehat{\mathcal{Z}}: \mathcal{Y}_{\text {big }}\right]$.

Remark 4.2.7. The big CM cycle arises from a morphism of Shimura varieties. Indeed, there is a morphism of Shimura data $\left(T_{\text {big }},\left\{h_{\text {big }}\right\}\right) \rightarrow(G, \mathcal{D})$, and the induced morphism of Shimura varieties sits in a commutative diagram of $E_{\Phi}$-stacks

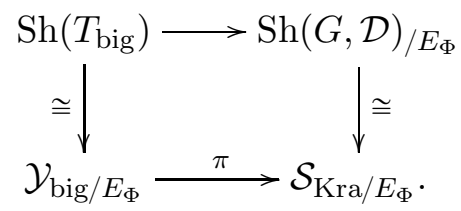

Proposition 4.2.8. The degree $\operatorname{deg}_{\mathbb{C}}\left(\mathcal{Y}_{\mathrm{big}}\right)$ of Theorem B satisfies

$$
\frac{1}{n} \cdot \operatorname{deg}_{\mathbb{C}}\left(\mathcal{Y}_{\text {big }}\right)=\frac{h_{\boldsymbol{k}}}{w_{\boldsymbol{k}}} \cdot \frac{\Lambda\left(0, \chi_{E}\right)}{2^{r-1}},
$$

where $r$ is the number of places of $F$ that ramify in $E$ (including all archimedean places).

Proof. It is clear from Proposition 4.2.4 that

$$
\frac{1}{n} \cdot \operatorname{deg}_{\mathbb{C}}\left(\mathcal{Y}_{\text {big }}\right)=\sum_{y \in \operatorname{Sh}\left(T_{\text {big }}\right)(\mathbb{C})} \frac{1}{|\operatorname{Aut}(y)|}=\frac{\left|T_{\text {big }}(\mathbb{Q}) \backslash T_{\text {big }}\left(\mathbb{A}_{f}\right) / K_{\text {big }}\right|}{\left|T_{\text {big }}(\mathbb{Q}) \cap K_{\text {big }}\right|} .
$$

Note that when we defined the degree on the left we counted the complex points of $\mathcal{Y}_{\text {big }}$ viewed as an $\mathcal{O}_{\boldsymbol{k}}$-stack, whereas in the middle expression we 


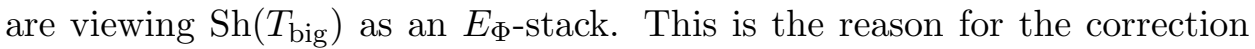
factor of $n=\left[E_{\Phi}: \boldsymbol{k}\right]$ on the left.

Let $E^{\prime} \subset E^{\times}$be the kernel of the norm map Nm : $E^{\times} \rightarrow F^{\times}$, and define

$$
\widehat{E}^{\prime} \subset \widehat{E}^{\times}, \quad \hat{\mathcal{O}}_{E}^{\prime} \subset \widehat{\mathcal{O}}_{E}^{\times}
$$

similarly. Note that $\mu(E)=E^{\prime} \cap \widehat{\mathcal{O}}_{E}^{\prime}$ is the group of roots of unity in $E$, whose order we denote by $w_{E}$. Using the isomorphism $T_{\text {big }}(\mathbb{Q}) \cong \boldsymbol{k}^{\times} \times E^{\prime}$ of Remark 4.1.1, we find

$$
\frac{\left|T_{\mathrm{big}}(\mathbb{Q}) \backslash T_{\mathrm{big}}\left(\mathbb{A}_{f}\right) / K_{\mathrm{big}}\right|}{\left|T_{\mathrm{big}}(\mathbb{Q}) \cap K_{\mathrm{big}}\right|}=\frac{h_{\boldsymbol{k}}}{w_{\boldsymbol{k}}} \cdot \frac{\left|E^{\prime} \backslash \widehat{E}^{\prime} / \widehat{\mathcal{O}}_{E}^{\prime}\right|}{w_{E}} .
$$

Denote by $C_{F}$ and $C_{E}$ the ideal class groups of $E$ and $F$, and by $\tilde{F}$ and $\tilde{E}$ their Hilbert class fields. As $E / F$ is ramified at all archimedean places, $\tilde{F} \cap E=F$, and the natural map

$$
\operatorname{Gal}(\tilde{E} / E) \rightarrow \operatorname{Gal}(\tilde{F} / F)
$$

is surjective. Hence, by class field theory, the norm

$$
\mathrm{Nm}: C_{E} \rightarrow C_{F}
$$

is surjective. Denote its kernel by $B$, so that we have a short exact sequence

$$
1 \rightarrow B \rightarrow C_{E} \stackrel{\mathrm{Nm}}{\longrightarrow} C_{F} \rightarrow 1 .
$$

Define a group

$$
\tilde{B}=E^{\times} \backslash\left\{(\mathfrak{B}, \beta): \begin{array}{c}
\mathfrak{B} \subset E \text { is a fractional } \mathcal{O}_{E} \text {-ideal, } \\
\beta \in F^{\times}, \text {and } \operatorname{Nm}(\mathfrak{B})=\beta \mathcal{O}_{F}
\end{array}\right\},
$$

where the action of $E^{\times}$is by $\alpha \cdot(\mathfrak{B}, \beta)=(\alpha \mathfrak{B}, \alpha \bar{\alpha} \beta)$. There is an evident short exact sequence

$$
1 \rightarrow \operatorname{Nm}\left(\mathcal{O}_{E}^{\times}\right) \backslash \mathcal{O}_{F}^{\times} \stackrel{\beta \mapsto\left(\mathcal{O}_{E}, \beta\right)}{\longrightarrow} \tilde{B} \rightarrow B \rightarrow 1 .
$$

Lemma 4.2.9. We have $\left[\mathcal{O}_{E}^{\times}: \operatorname{Nm}\left(\mathcal{O}_{E}^{\times}\right)\right]=2^{n-1} w_{E}$.

Proof. Let $Q=\left[\mathcal{O}_{E}^{\times}: \mu(E) \mathcal{O}_{F}^{\times}\right]$. If $Q=1$ then

$$
\left[\operatorname{Nm}\left(\mathcal{O}_{E}^{\times}\right): \mathcal{O}_{F}^{\times, 2}\right]=1 \quad \text { and }\left[\mathcal{O}_{E}^{\times}: \mathcal{O}_{F}^{\times}\right]=\frac{1}{2} \cdot w_{E},
$$

and so

$$
\left[\mathcal{O}_{F}^{\times}: \operatorname{Nm}\left(\mathcal{O}_{E}^{\times}\right)\right]=\left[\mathcal{O}_{F}^{\times}: \mathcal{O}_{F}^{\times, 2}\right]=2^{n}=\frac{2^{n-1} w_{E}}{\left[\mathcal{O}_{E}^{\times}: \mathcal{O}_{F}^{\times}\right]},
$$

where the middle equality follows from Dirichlet's unit theorem.

If $Q>1$ then [Was82, Theorem 4.12] and its proof show that $Q=2$, and that the image of the map $\phi: \mathcal{O}_{E}^{\times} \rightarrow \mathcal{O}_{E}^{\times}$defined by $\phi(x)=x / \bar{x}$ is the index two subgroup $\phi\left(\mathcal{O}_{E}^{\times}\right)=\mu(E)^{2} \subset \mu(E)$. From this it follows easily that

$$
\left[\operatorname{Nm}\left(\mathcal{O}_{E}^{\times}\right): \mathcal{O}_{F}^{\times, 2}\right]=2 \text { and }\left[\mathcal{O}_{E}^{\times}: \mathcal{O}_{F}^{\times}\right]=w_{E},
$$


and so

$$
\left[\mathcal{O}_{F}^{\times}: \operatorname{Nm}\left(\mathcal{O}_{E}^{\times}\right)\right]=\frac{1}{2} \cdot\left[\mathcal{O}_{F}^{\times}: \mathcal{O}_{F}^{\times, 2}\right]=2^{n-1}=\frac{2^{n-1} w_{E}}{\left[\mathcal{O}_{E}^{\times}: \mathcal{O}_{F}^{\times}\right]}
$$

Combining the information we have so far gives

$$
|\tilde{B}|=\left[\mathcal{O}_{F}^{\times}: \operatorname{Nm}\left(\mathcal{O}_{E}^{\times}\right)\right] \cdot|B|=\frac{2^{n-1} w_{E}}{\left[\mathcal{O}_{E}^{\times}: \mathcal{O}_{F}^{\times}\right]} \cdot \frac{\left|C_{E}\right|}{\left|C_{F}\right|}=w_{E} \cdot \Lambda\left(0, \chi_{E}\right),
$$

where the final equality is a consequence of Dirichlet's class number formula.

Lemma 4.2.10. There is an exact sequence

$$
1 \rightarrow E^{\prime} \backslash \widehat{E}^{\prime} / \widehat{\mathcal{O}}_{E}^{\prime} \rightarrow \tilde{B} \rightarrow\{ \pm 1\}^{r} \rightarrow\{ \pm 1\} \rightarrow 1 .
$$

Proof. Every $x \in \widehat{E}^{\prime}$ determines a fractional $\mathcal{O}_{E}$-ideal $\mathfrak{B}=x \mathcal{O}_{E}$ with $\operatorname{Nm}(\mathfrak{B})=\mathcal{O}_{F}$, and the rule $x \mapsto(\mathfrak{B}, 1)$ is easily seen to define an injection

$$
E^{\prime} \backslash \widehat{E}^{\prime} / \widehat{\mathcal{O}}_{E}^{\prime} \rightarrow \tilde{B}
$$

Given a $(\mathfrak{B}, \beta) \in \tilde{B}$, consider the elements $\chi_{E, v}(\beta) \in\{ \pm 1\}$ as $v$ runs over all places of $F$. If $v$ is split in $E$ then certainly $\chi_{E, v}(\beta)=1$. If $v$ is inert in $E$ then $\operatorname{Nm}(\mathfrak{B})=\beta \mathcal{O}_{F}$ implies that $\chi_{E, v}(\beta)=1$. As the product over all $v$ of $\chi_{E, v}(\beta)$ is equal to 1 , we see that sending $(\mathfrak{B}, \beta)$ to the tuple of $\chi_{E, v}(\beta)$ with $v$ ramified in $E$ defines a homomorphism

$$
\tilde{B} \rightarrow \operatorname{ker}\left(\{ \pm 1\}^{r} \stackrel{\text { product }}{\longrightarrow}\{ \pm 1\}\right) .
$$

To see that (4.2.5) is surjective, fix a tuple $\left(\epsilon_{v}\right)_{v} \in\{ \pm 1\}^{r}$ indexed by the places of $F$ ramified in $E$, and assume that $\prod_{v} \epsilon_{v}=1$. Let $b \in \mathbb{A}_{F}^{\times}$be any idele satisfying:

- If $v$ is ramified in $E$ then $\chi_{E, v}\left(b_{v}\right)=\epsilon_{v}$.

- If $v$ is a finite place of $F$ then $b_{v} \in \mathcal{O}_{F, v}^{\times}$.

The second condition implies that $\chi_{E, v}\left(b_{v}\right)=1$ whenever $v$ is unramified in $E$, and hence

$$
\chi_{E}(b)=\prod_{v} \epsilon_{v}=1 .
$$

Thus $b$ lies in the kernel of the reciprocity map

$$
\mathbb{A}_{F}^{\times} \rightarrow F^{\times} \backslash \mathbb{A}_{F}^{\times} / \mathrm{Nm}\left(\mathbb{A}_{E}^{\times}\right) \cong \operatorname{Gal}(E / F),
$$

and so can be factored as $b=\beta^{-1} x \bar{x}$ for some $\beta \in F^{\times}$and $x \in \mathbb{A}_{E}^{\times}$. Setting $\mathfrak{B}=x \mathcal{O}_{E}$, the pair $(\mathfrak{B}, \beta) \in \tilde{B}$ maps to $\left(\epsilon_{v}\right)_{v}$ under (4.2.5).

It only remains to show that the image of (4.2.4) is equal to the kernel of (4.2.5). It is clear from the definitions that the composition

$$
E^{\prime} \backslash \widehat{E}^{\prime} / \widehat{\mathcal{O}}_{E}^{\prime} \rightarrow \tilde{B} \rightarrow\{ \pm 1\}^{r}
$$

is trivial, proving one inclusion. For the other inclusion, suppose $(\mathfrak{B}, \beta) \in \tilde{B}$ lies in the kernel of (4.2.5). We have already seen that this implies that 
$\beta \in F^{\times}$satisfies $\chi_{E, v}(\beta)=1$ for every place $v$ of $F$, and so $\beta$ is a norm from $E$ everywhere locally. By the Hasse-Minkowski theorem, $\beta$ is a norm globally, say $\beta=\alpha \bar{\alpha}$ with $\alpha \in E^{\times}$. In the group $\tilde{B}$, we therefore have the relation

$$
(\mathfrak{B}, \beta)=\alpha^{-1}(\mathfrak{B}, \beta)=(\mathfrak{A}, 1)
$$

for a fractional $\mathcal{O}_{E}$-ideal $\mathfrak{A}=\alpha^{-1} \mathfrak{B}$ satisfying $\operatorname{Nm}(\mathfrak{A})=\mathcal{O}_{F}$. Any such $\mathfrak{A}$ has the form $\mathfrak{A}=x \mathcal{O}_{E}$ for some $x \in \widehat{E}^{\prime}$, proving that $(\mathfrak{B}, \beta)$ lies in the image of (4.2.4).

Combining the lemma with (4.2.3) gives

$$
\frac{\left|E^{\prime} \backslash \hat{E}^{\prime} / \widehat{\mathcal{O}}_{E}^{\prime}\right|}{w_{E}}=\frac{|\tilde{B}|}{2^{r-1} w_{E}}=\frac{\Lambda\left(0, \chi_{E}\right)}{2^{r-1}}
$$

and combining this with (4.2.2) completes the proof of Proposition 4.2.8,

Proposition 4.2.11. Assume that the discriminants of $\boldsymbol{k}$ and $F$ are relatively prime. The constant term (1.4.2) satisfies

$$
\left[\hat{\mathcal{Z}}_{\mathrm{Kra}}^{\text {tot }}(0): \mathcal{Y}_{\text {big }}\right]=-\left[\widehat{\boldsymbol{\omega}}: \mathcal{Y}_{\text {big }}\right] \text {. }
$$

Proof. The stated equality is equivalent to

$$
\left[(\operatorname{Exc},-\log (D)): \mathcal{Y}_{\mathrm{big}}\right]=0
$$

and so it suffices to prove

$$
\left[(0, \log (D)): \mathcal{Y}_{\text {big }}\right]=\operatorname{deg}_{\mathbb{C}}\left(\mathcal{Y}_{\text {big }}\right) \cdot \log (D)=\left[(\text { Exc, } 0): \mathcal{Y}_{\text {big }}\right]
$$

The first equality is clear from the definitions. To prove the second equality, we first argue that

$$
\mathcal{Y}_{\text {big }} \times \mathcal{S}_{\text {Kra }} \operatorname{Exc}=\mathcal{Y}_{\text {big }} \times{ }_{\operatorname{Spec}\left(\mathcal{O}_{\boldsymbol{k}}\right)} \operatorname{Spec}\left(\mathcal{O}_{\boldsymbol{k}} / \mathfrak{d}_{\boldsymbol{k}}\right),
$$

as in the proof of Proposition 2.2.10,

The inclusion $\subset$ of (4.2.6) is again clear from

$$
\operatorname{Exc} \subset \mathcal{S}_{\text {Kra }} \times{ }_{\operatorname{Spec}\left(\mathcal{O}_{\boldsymbol{k}}\right)} \operatorname{Spec}\left(\mathcal{O}_{\boldsymbol{k}} / \mathfrak{d}_{\boldsymbol{k}}\right) .
$$

Recall that $\mathcal{Y}_{\text {big }} \rightarrow \operatorname{Spec}\left(\mathcal{O}_{\Phi}\right)$ is étale. Our hypothesis on the discriminants of $\boldsymbol{k}$ and $F$ implies that $\operatorname{Spec}\left(\mathcal{O}_{\Phi}\right) \rightarrow \operatorname{Spec}\left(\mathcal{O}_{\boldsymbol{k}}\right)$ is étale at all primes dividing $\mathfrak{d}_{k}$, and hence the same is true for $\mathcal{Y}_{\text {big }} \rightarrow \operatorname{Spec}\left(\mathcal{O}_{k}\right)$. This implies that the right hand side of (4.2.6) is reduced, and hence so is the left hand side. To prove equality in (4.2.6), it therefore suffices to prove the inclusion $\supset$ on the level of geometric points.

Suppose $\mathfrak{p} \mid \mathfrak{d}_{k}$ is prime, and let $\mathbb{F}_{\mathfrak{p}}^{\text {alg }}$ be an algebraic closure of its residue field. Suppose that $y \in \mathcal{Y}_{\text {big }}\left(\mathbb{F}_{\mathfrak{p}}^{\text {alg }}\right)$ corresponds to the pair $\left(A_{0}, A\right)$, so that $A \in \mathcal{C} \mathcal{M}_{\Phi}\left(\mathbb{F}_{\mathfrak{p}}^{\text {alg }}\right)$. Let $W$ be the completed étale local ring of the geometric point

$$
\operatorname{Spec}\left(\mathbb{F}_{\mathfrak{p}}^{\text {alg }}\right) \stackrel{y}{\rightarrow} \mathcal{Y}_{\text {big }} \rightarrow \operatorname{Spec}\left(\mathcal{O}_{\Phi}\right) .
$$

More concretely, $W$ is the completion of the maximal unramified extension of $\mathcal{O}_{\boldsymbol{k}, \mathfrak{p}}$, equipped with an injective ring homomorphism $\mathcal{O}_{\Phi} \rightarrow W$. Let $\mathbb{C}_{\mathfrak{p}}$ 
be the completion of an algebraic closure of the fraction field of $W$, and fix an isomorphism of $E_{\Phi}$-algebras $\mathbb{C} \cong \mathbb{C}_{\mathfrak{p}}$.

For every $\varphi \in \Phi$ the induced map $\mathcal{O}_{E} \rightarrow \mathbb{C} \cong \mathbb{C}_{\mathfrak{p}}$ takes values in the subring $W$, and the induced map

$$
\mathcal{O}_{E} \otimes_{\mathbb{Z}} W \rightarrow \prod_{\varphi \in \Phi} W
$$

is surjective (by our hypothesis that $\boldsymbol{k}$ and $F$ have relatively prime discriminants). Denote its kernel by $J_{\Phi} \subset \mathcal{O}_{E} \otimes_{\mathbb{Z}} W$, and define an $\mathcal{O}_{E} \otimes_{\mathbb{Z}} W$-module

$$
\operatorname{Lie}_{\Phi}=\left(\mathcal{O}_{E} \otimes_{\mathbb{Z}} W\right) / J_{\Phi} \cong \prod_{\varphi \in \Phi} W .
$$

As in the proof of [How15, Lemma 4.1.2], there is an isomorphism of $\mathcal{O}_{E} \otimes_{\mathbb{Z}}$ $\mathbb{F}_{\mathfrak{p}}^{\text {alg }}$-modules

$$
\operatorname{Lie}(A) \cong \operatorname{Lie}_{\Phi} \otimes_{W} \mathbb{F}_{\mathfrak{p}}^{\text {alg }} \cong \prod_{\varphi \in \Phi} \mathbb{F}_{\mathfrak{p}}^{\text {alg }}
$$

Let $\delta \in \mathcal{O}_{\boldsymbol{k}}$ be a square root of $-D$. As the image of $\delta$ under

$$
\mathcal{O}_{E} \stackrel{\varphi}{\rightarrow} W \rightarrow \mathbb{F}_{\mathfrak{p}}^{\text {alg }}
$$

is 0 for every $\varphi \in \Phi$, it follows from what was said above that $\delta$ annihilates $\operatorname{Lie}(A)$. Exactly as in the proof of Proposition 2.2.10, this implies that the image of $y$ under $\mathcal{Y}_{\text {big }} \rightarrow \mathcal{S}_{\text {Kra }}$ lies on the exceptional divisor. This completes the proof of (4.2.6), and the remainder of the proof is exactly as in Proposition 2.2.10.

4.3. A generalized $L$-function. The action $i_{E}: \mathcal{O}_{E} \rightarrow \operatorname{End}_{\mathcal{O}_{k}}(\mathfrak{a})$ makes

$$
L=\operatorname{Hom}_{\mathcal{O}_{\boldsymbol{k}}}\left(\mathfrak{a}_{0}, \mathfrak{a}\right)
$$

into a projective $\mathcal{O}_{E}$-module of rank one, and the $\mathcal{O}_{\boldsymbol{k}}$-hermitian form on $L$ defined by [BHKRYa, (2.1.5)] satisfies $\left\langle\alpha x_{1}, x_{2}\right\rangle=\left\langle x_{1}, \bar{\alpha} x_{2}\right\rangle$ for all $\alpha \in \mathcal{O}_{E}$ and $x_{1}, x_{2} \in L$. It is a formal consequence of this that the $E$-vector space $\mathscr{V}=L \otimes_{\mathbb{Z}} \mathbb{Q}$ carries an $E$-hermitian form

$$
\langle-,-\rangle_{\text {big }}: \mathscr{V} \times \mathscr{V} \rightarrow E,
$$

uniquely determined by the property

$$
\left\langle x_{1}, x_{2}\right\rangle=\operatorname{Tr}_{E / \boldsymbol{k}}\left\langle x_{1}, x_{2}\right\rangle_{\text {big }} .
$$

This hermitian form has signature $(0,1)$ at $\left.\varphi^{\mathrm{sp}}\right|_{F}$, and signature $(1,0)$ at all other archimedean places of $F$.

From the $E$-hermitian form we obtain an $F$-valued quadratic form $\mathscr{Q}(x)=$ $\langle x, x\rangle_{\text {big }}$ on $\mathscr{V}$ with signature $(0,2)$ at $\left.\varphi^{\mathrm{sp}}\right|_{F}$, and signature $(2,0)$ at all other archimedean places of $F$. The $\mathbb{Q}$-quadratic form

$$
Q(x)=\operatorname{Tr}_{F / \mathbb{Q}} \mathscr{Q}(x)
$$

is $\mathbb{Z}$-valued on $L \subset \mathscr{V}$, and agrees with the quadratic form of $₫ 2.3$. Let

$$
\omega_{L}: \mathrm{SL}_{2}(\mathbb{Z}) \rightarrow \operatorname{Aut}_{\mathbb{C}}\left(S_{L}\right)
$$


be the Weil representation on the space $S_{L}=\mathbb{C}\left[L^{\prime} / L\right]$, where $L^{\prime}=\mathfrak{d}_{k}^{-1} L$ is the dual lattice of $L$ relative to the $\mathbb{Z}$-bilinear form (2.3.1).

Write each $\vec{\tau} \in F_{\mathbb{C}}$ in the form $\vec{\tau}=\vec{u}+i \vec{v}$ with $\vec{u}, \vec{v} \in F_{\mathbb{R}}$, and set

$$
\mathcal{H}_{F}=\left\{\vec{\tau} \in F_{\mathbb{C}}: \vec{v} \text { is totally positive }\right\} .
$$

Every Schwartz function $\phi \in S(\widehat{\mathscr{V}})$ determines an incoherent Hilbert modular Eisenstein series

$$
E(\vec{\tau}, s, \phi)=\sum_{\alpha \in F} E_{\alpha}(\vec{v}, s, \phi) \cdot q^{\alpha}
$$

on $\mathcal{H}_{F}$, as in [BKY12, (4.4)] and [AGHMP18, $\left.\S 6.1\right]$. If we identify

$$
S_{L}=\mathbb{C}\left[L^{\prime} / L\right] \subset S(\widehat{\mathscr{V}})
$$

as the space of $\widehat{L}$-invariant functions supported on $\widehat{L}^{\prime}$, then (4.3.2) can be viewed as a function $E(\vec{\tau}, s)$ on $\mathcal{H}_{F}$ taking values in the complex dual $S_{L}^{\vee}$.

We quickly recall the construction of (4.3.2). If $v$ is an arichmedean place of $F$, denote by $\left(\mathscr{C}_{v}, \mathscr{Q}_{v}\right)$ the unique positive definite rank 2 quadratic space over $F_{v}$. Set $\mathscr{C}_{\infty}=\prod_{v \mid \infty} \mathscr{C}_{v}$. The rank 2 quadratic space

$$
\mathscr{C}=\mathscr{C}_{\infty} \times \widehat{\mathscr{V}}
$$

over $\mathbb{A}_{F}$ is incoherent, in the sense that it is not the adelization of any $F$ quadratic space. In fact, $\mathscr{C}$ is isomorphic to $\mathscr{V}$ everywhere locally, except at the unique archimedean place $\left.\varphi^{\mathrm{sp}}\right|_{F}$ at which $\mathscr{V}$ is negative definite.

Let $\psi_{\mathbb{Q}}: \mathbb{Q} \backslash \mathbb{A} \rightarrow \mathbb{C}^{\times}$be the standard additive character, and define

$$
\psi_{F}: F \backslash \mathbb{A}_{F} \rightarrow \mathbb{C}^{\times}
$$

by $\psi_{F}=\psi_{\mathbb{Q}} \circ \operatorname{Tr}_{F / \mathbb{Q}}$. Denote by $I\left(s, \chi_{E}\right)$ the degenerate principal series representation of $\mathrm{SL}_{2}\left(\mathbb{A}_{F}\right)$ induced from the character $\left.\left.\chi_{E}\right|^{\cdot}\right|^{s}$ on the subgroup $B \subset \mathrm{SL}_{2}$ of upper triangular matrices. Thus $I\left(s, \chi_{E}\right)$ consists of all smooth functions $\Phi(g, s)$ on $\mathrm{SL}_{2}\left(\mathbb{A}_{F}\right)$ satisfying the transformation law

$$
\Phi\left(\left(\begin{array}{cc}
a & b \\
& a^{-1}
\end{array}\right) g, s\right)=\chi_{E}(a)|a|^{s+1} \Phi(g, s) .
$$

The Weil representation $\omega_{\mathscr{C}}$ determined by the character $\psi_{F}$ defines an action of $\mathrm{SL}_{2}\left(\mathbb{A}_{F}\right)$ on $S(\mathscr{C})$, and for any Schwartz function

$$
\phi_{\infty} \otimes \phi \in S\left(\mathscr{C}_{\infty}\right) \otimes S(\widehat{\mathscr{V}}) \cong S(\mathscr{C})
$$

the function

$$
\Phi(g, 0)=\omega_{\mathscr{C}}(g)\left(\phi_{\infty} \otimes \phi\right)(0)
$$

lies in the induced representation $I\left(0, \chi_{E}\right)$. It extends uniquely to a standard section $\Phi(g, s)$ of $I\left(s, \chi_{E}\right)$, which determines an Eisenstein series

$$
E\left(g, s, \phi_{\infty} \otimes \phi\right)=\sum_{\gamma \in B(F) \backslash \mathrm{SL}_{2}(F)} \Phi(\gamma g, s)
$$

in the variable $g \in \mathrm{SL}_{2}\left(\mathbb{A}_{F}\right)$. 
We always choose $\phi \in S_{L} \subset S(\mathscr{V})$, and take the archimedean component $\phi_{\infty}$ of our Schwartz function to be the Gaussian distribution

$$
\phi_{\infty}^{\mathbf{1}}=\otimes \phi_{v}^{\mathbf{1}} \in \underset{v \mid \infty}{\bigotimes} S\left(\mathscr{C}_{v}\right)
$$

defined by $\phi_{v}^{\mathbf{1}}(x)=e^{-2 \pi \mathscr{Q}_{v}(x)}$, so that the resulting Eisenstein series

$$
E(\vec{\tau}, s, \phi)=\frac{1}{\sqrt{\mathrm{Nm}(\vec{v})}} \cdot E\left(g_{\vec{\tau}}, s, \phi_{\infty}^{\mathbf{1}} \otimes \phi\right)
$$

has parallel weight 1 . Here

$$
g_{\vec{\tau}}=\left(\begin{array}{ll}
1 & \vec{u} \\
0 & 1
\end{array}\right)\left(\begin{array}{cc}
\sqrt{\vec{v}} & \\
& 1 / \sqrt{\vec{v}}
\end{array}\right) \in \mathrm{SL}_{2}\left(F_{\mathbb{R}}\right)
$$

and $\mathrm{Nm}: F_{\mathbb{R}}^{\times} \rightarrow \mathbb{R}^{\times}$is the norm.

A choice of ordering of the embeddings $F \rightarrow \mathbb{R}$ fixes an isomorphism of $\mathcal{H}_{F}$ with the $n$-fold product of the complex upper half-plane with itself, and the diagonal inclusion $\mathcal{H} \hookrightarrow \mathcal{H}_{F}$ is independent of the choice of ordering. By restricting our Eisenstein series to the diagonal we obtain an $S_{L}^{\vee}$-valued function

$$
E(\tau, s)=\left.E(\vec{\tau}, s)\right|_{\mathcal{H}}
$$

in the variable $\tau \in \mathcal{H}$, which transforms like a modular form of weight $n$ and representation $\omega_{L}^{\vee}$ under the full modular group $\mathrm{SL}_{2}(\mathbb{Z})$.

Given a cusp form $\tilde{g} \in S_{n}\left(\bar{\omega}_{L}\right)$ valued in $S_{L}$, consider the Petersson inner product

$$
\langle E(s), \tilde{g}\rangle_{\mathrm{Pet}}=\int_{\mathrm{SL}_{2}(\mathbb{Z}) \backslash \mathcal{H}}\{\overline{\tilde{g}(\tau)}, E(\tau, s)\} \frac{d u d v}{v^{2-n}},
$$

where $\{\cdot, \cdot\}: S_{L} \times S_{L}^{\vee} \rightarrow \mathbb{C}$ is the tautological pairing. This is an unnormalized version of the generalized $L$-function

$$
\mathcal{L}(s, \tilde{g})=\Lambda\left(s+1, \chi_{E}\right) \cdot\langle E(s), \tilde{g}\rangle_{\mathrm{Pet}}
$$

of [BKY12, (1.2)] or [AGHMP18, $§ 6.3]$.

Let $F_{+} \subset F$ be the subset of totally positive elements. The Eisenstein series $E(\vec{\tau}, s)$ satisfies a functional equation in $s \mapsto-s$, forcing it to vanish at $s=0$. As in [BKY12, Proposition 4.6] and [AGHMP18, §6.2], we can extract from the central derivative $E^{\prime}(\vec{\tau}, 0)$ a formal $q$-expansion

$$
a_{F}(0)+\sum_{\alpha \in F_{+}} a_{F}(\alpha) \cdot q^{\alpha}
$$

If $\alpha \in F_{+}$then $E_{\alpha}^{\prime}(\vec{v}, 0, \phi)$ is independent of $\vec{v}$, and we define $a_{F}(\alpha) \in S_{L}^{\vee}$ by

$$
a_{F}(\alpha, \phi)=\Lambda\left(0, \chi_{E}\right) \cdot E_{\alpha}^{\prime}(\vec{v}, 0, \phi) .
$$

We define $a_{F}(0) \in S_{L}^{\vee}$ by

$$
a_{F}(0, \phi)=\Lambda(0, \chi) \cdot E_{0}^{\prime}(\vec{v}, 0, \phi)-\Lambda\left(0, \chi_{E}\right) \cdot \phi(0) \log \operatorname{Nm}(\vec{v}) .
$$

Again, this is independent of $\vec{v}$. 
Remark 4.3.1. For notational simplicity, we often denote by $a_{F}(\alpha, \mu)$ the value of $a_{F}(\alpha): S_{L} \rightarrow \mathbb{C}$ at the characteristic function of a coset $\mu \in L^{\prime} / L$.

For any nonzero $\alpha \in F$, define

$$
\operatorname{Diff}(\mathscr{C}, \alpha)=\left\{\text { places } v \text { of } F: \mathscr{C}_{v} \text { does not represent } \alpha\right\} .
$$

This is a finite set of odd cardinality, and any $v \in \operatorname{Diff}(\mathscr{C}, \alpha)$ is necessarily nonsplit in $E$. We are really only interested in this set when $\alpha \in F_{+}$. As $\mathscr{C}$ is positive definite at all archimedean places, for such $\alpha$ we have

$$
\operatorname{Diff}(\mathscr{C}, \alpha)=\left\{\text { primes } \mathfrak{p} \subset \mathcal{O}_{F}: \mathscr{V}_{\mathfrak{p}} \text { does not represent } \alpha\right\}
$$

We will need explicit formulas for all $a_{F}(\alpha, \mu)$ with $\alpha \in F_{+}$, but only for the trivial coset $\mu=0$. These are provided by the following proposition.

Proposition 4.3.2. Suppose $\alpha \in F_{+}$.

(1) If $|\operatorname{Diff}(\mathscr{C}, \alpha)|>1$ then $a_{F}(\alpha)=0$.

(2) If $\operatorname{Diff}(\mathscr{C}, \alpha)=\{\mathfrak{p}\}$, then

$$
a_{F}(\alpha, 0)=-2^{r-1} \cdot \rho\left(\alpha \mathfrak{d}_{F} \mathfrak{p}^{-\epsilon_{\mathfrak{p}}}\right) \cdot \operatorname{ord}_{\mathfrak{p}}\left(\alpha \mathfrak{p} \mathfrak{d}_{F}\right) \cdot \log (\mathrm{N}(\mathfrak{p})),
$$

where the notation is as follows: $r$ is the number of places of $F$ ramified in $E$ (including all archimedean places), $\mathfrak{d}_{F} \subset \mathcal{O}_{F}$ is the different of $F$, and

$$
\epsilon_{\mathfrak{p}}=\left\{\begin{array}{l}
1 \quad \text { if } \mathfrak{p} \text { is inert in } E \\
0 \quad \text { if } \mathfrak{p} \text { is ramified in } E .
\end{array}\right.
$$

Moreover, for any fractional $\mathcal{O}_{F}$-ideal $\mathfrak{b} \subset F$ we have set

$$
\rho(\mathfrak{b})=\mid\left\{\text { ideals } \mathfrak{B} \subset \mathcal{O}_{E}: \mathfrak{B} \overline{\mathfrak{B}}=\mathfrak{b} \mathcal{O}_{E}\right\} \mid .
$$

In particular, $\rho(\mathfrak{b})=0$ unless $\mathfrak{b} \subset \mathcal{O}_{F}$.

Proof. Up to a change of notation, this is [How12, Proposition 4.2.1], whose proof amounts to collecting together calculations of [Yan05]. More general formulas can be found in [AGHMP18, §7.1] and [HY12, §4.6].

Proposition 4.3.3. Assume that the discriminants of $\boldsymbol{k}$ and $F$ are relatively prime. For any $\mu \in L^{\prime} / L$ we have

$$
a_{F}(0, \mu)= \begin{cases}-2 \Lambda^{\prime}\left(0, \chi_{E}\right) & \text { if } \mu=0 \\ 0 & \text { otherwise. }\end{cases}
$$

Proof. Let $\Phi_{\mu}=\prod_{\mathfrak{p}} \Phi_{\mu, \mathfrak{p}}$ be the standard section of $I\left(s, \chi_{E}\right)$ determined by the characteristic function $\phi_{\mu} \in S_{L} \subset S(\mathscr{V})$ of $\mu \in L^{\prime} / L$. According to [AGHMP18, Proposition 6.2.3], we then have

$$
a_{F}(0, \mu)=-2 \phi_{\mu}(0) \Lambda^{\prime}\left(0, \chi_{E}\right)-\left.\Lambda\left(0, \chi_{E}\right) \cdot \frac{d}{d s}\left(\prod_{\mathfrak{p}} M_{\mathfrak{p}}\left(s, \phi_{\mu}\right)\right)\right|_{s=0}
$$


where the product is over all finite places $\mathfrak{p}$ of $F$, and the local factors on the right have the form

$$
M_{\mathfrak{p}}\left(s, \phi_{\mu}\right)=c_{\mathfrak{p}} \cdot \frac{L_{\mathfrak{p}}\left(s+1, \chi_{E}\right)}{L_{\mathfrak{p}}\left(s, \chi_{E}\right)} \cdot W_{0, \mathfrak{p}}\left(s, \Phi_{\mu}\right)
$$

for some constants $c_{\mathfrak{p}}$ independent of $s$. Here, setting

$$
w=\left(\begin{array}{cc}
0 & -1 \\
1 & 0
\end{array}\right), \quad n(b)=\left(\begin{array}{ll}
1 & b \\
0 & 1
\end{array}\right),
$$

the function

$$
W_{0, \mathfrak{p}}\left(s, \Phi_{\mu}\right)=\int_{F_{\mathfrak{p}}} \Phi_{\mu, \mathfrak{p}}(w n(b), s) d b
$$

is the value of the local Whittaker function $W_{0, \mathfrak{p}}\left(g, s, \Phi_{\mu}\right)$ at the identity in $\mathrm{SL}_{2}\left(F_{\mathfrak{p}}\right)$. Our goal is to prove that $M_{\mathfrak{p}}\left(s, \phi_{\mu}\right)$ is independent of $s$, and hence both the particular value of $c_{\mathfrak{p}}$ and the choice of Haar measure on $F_{\mathfrak{p}}$ are irrelevant to us.

Fix a prime $\mathfrak{p} \subset \mathcal{O}_{F}$, and let $p$ be the rational prime below it. We may identify $\mathscr{V}_{\mathfrak{p}} \cong E_{\mathfrak{p}}$ in such a way that $L_{\mathfrak{p}} \cong \mathcal{O}_{E, \mathfrak{p}}$, and so that the $F_{\mathfrak{p}}$-valued quadratic form $\mathscr{Q}$ on $\mathscr{V}_{\mathfrak{p}} \cong E_{\mathfrak{p}}$ becomes

$$
\mathscr{Q}(x)=\beta x \bar{x}
$$

for some $\beta \in F_{\mathfrak{p}} \times$. If $\mathfrak{d}_{F}$ denotes the different of $F / \mathbb{Q}$, then

$$
\beta \mathcal{O}_{F, \mathfrak{p}}=\mathfrak{d}_{F}^{-1} \mathcal{O}_{F, \mathfrak{p}} .
$$

Indeed, let $\mathfrak{d}_{E}$ be the different of $E / \mathbb{Q}$. The lattice $L_{\mathfrak{p}}^{\prime}=\mathfrak{d}_{\boldsymbol{k}}^{-1} \mathcal{O}_{E, \mathfrak{p}}$ is the dual lattice of $\mathcal{O}_{E, \mathfrak{p}}$ relative to the $\mathbb{Q}_{p}$-bilinear form $[x, y]=\operatorname{Tr}_{E_{\mathfrak{p}} / \mathbb{Q}_{p}}(\beta x \bar{y})$, which implies the first equality in

$$
\beta^{-1} \mathcal{O}_{E, \mathfrak{p}}=\mathfrak{d}_{E} \mathfrak{d}_{\boldsymbol{k}}^{-1} \mathcal{O}_{E, \mathfrak{p}}=\mathfrak{d}_{F} \mathcal{O}_{E, \mathfrak{p}}
$$

The second equality is a consequence of our assumption that the discriminants of $\boldsymbol{k}$ and $F$ are relatively prime.

If we endow $\mathscr{V}_{\mathfrak{p}}=E_{\mathfrak{p}}$ with the rescaled quadratic form

$$
\mathscr{Q}^{\sharp}(x) \stackrel{\text { def }}{=} \beta^{-1} \mathscr{Q}(x)=x \bar{x},
$$

and define a new additive character

$$
\psi_{F, \mathfrak{p}}^{\sharp}(x) \stackrel{\text { def }}{=} \psi_{F, \mathfrak{p}}(\beta x)
$$

(unramified by (4.3.7)), we obtain a new Weil representation

$$
\omega^{\sharp}: \operatorname{SL}_{2}\left(F_{\mathfrak{p}}\right) \rightarrow \operatorname{Aut}\left(S\left(\mathscr{V}_{\mathfrak{p}}\right)\right),
$$

and hence, as in (4.3.3), a function

$$
S\left(\mathscr{V}_{\mathfrak{p}}\right) \stackrel{\phi \mapsto \Phi_{\mathfrak{p}}^{\sharp}(s, g)}{\longrightarrow} I_{\mathfrak{p}}\left(s, \chi_{E}\right)
$$

defined by first setting $\Phi_{\mathfrak{p}}^{\sharp}(0, g)=\omega^{\sharp}(g) \phi(0)$, and then extending to a standard section. 
The local Schwartz function $\phi_{\mu, \mathfrak{p}} \in S\left(\mathscr{V}_{\mathfrak{p}}\right)$ now determines a standard section $\Phi_{\mu, \mathfrak{p}}^{\sharp}(g, s)$ of $I_{\mathfrak{p}}\left(s, \chi_{E}\right)$, and explicit formulas for the Weil representation, as in [HY12, (4.2.1)], show that

$$
\int_{F_{\mathfrak{p}}} \Phi_{\mu, \mathfrak{p}}(w n(b), s) d b=\int_{F_{\mathfrak{p}}} \Phi_{\mu, \mathfrak{p}}^{\sharp}(w n(b), s) d b .
$$

What our discussion shows is that there is no harm in rescaling the quadratic form on $\mathscr{V}_{\mathfrak{p}}$ to make $\beta=1$, and simultaneously modifying the additive character $\psi_{F, \mathfrak{p}}$ to make it unramified.

After this rescaling, one can easily deduce explicit formulas for $W_{0, \mathfrak{p}}\left(s, \Phi_{\mu}\right)$ from the literature. Indeed, if the local component $\mu_{\mathfrak{p}} \in L_{\mathfrak{p}}^{\prime} / L_{\mathfrak{p}}$ is zero, then the calculations found in [Yan05, §2] imply that

$$
W_{0, \mathfrak{p}}\left(s, \Phi_{\mu}\right)=\frac{L_{\mathfrak{p}}\left(s, \chi_{E}\right)}{L_{\mathfrak{p}}\left(s+1, \chi_{E}\right)}
$$

up to scaling by a nonzero constant independent of $s$. If instead $\mu_{\mathfrak{p}} \neq 0$ then $\mathfrak{p}$ is ramified in $E$ (and in particular $p>2$ ), and it follows from the calculations found in the proof of [HY12, Proposition 4.6.4] that $W_{0, \mathfrak{p}}\left(s, \Phi_{\mu}\right)=0$. In any case (4.3.6) is independent of $s$ for every $\mathfrak{p}$, and so the derivative in (4.3.5) vanishes.

4.4. A preliminary central derivative formula. The entirety of 44.4 is devoted to proving Theorem 4.4.1, which a big CM analogue of Theorem 2.4.1. The proof will make essential use of the calculations of [How12, How15, BKY12.

We assume $n \geqslant 3$ throughout 44.4 . This allows us to make use of the distinguished harmonic forms

$$
f_{m} \in H_{2-n}\left(\bar{\omega}_{L}\right)^{\Delta}
$$

(for $m>0$ ) characterized by (2.5.1).

Theorem 4.4.1. Assume that the discriminants of $\boldsymbol{k} / \mathbb{Q}$ and $F / \mathbb{Q}$ are odd and relatively prime, and fix a positive integer $m$. If $f=f_{m}$ is the harmonic form above, and $\widehat{\mathcal{Z}}$ is the linear function (2.4.1), then

$$
\frac{n \cdot\left[\widehat{\mathcal{Z}}(f): \mathcal{Y}_{\text {big }}\right]}{\operatorname{deg}_{\mathbb{C}}\left(\mathcal{Y}_{\text {big }}\right)}+2 c_{f}^{+}(0,0) \frac{\Lambda^{\prime}\left(0, \chi_{E}\right)}{\Lambda\left(0, \chi_{E}\right)}=-\left.\frac{d}{d s}\langle E(s), \xi(f)\rangle_{\text {Pet }}\right|_{s=0} .
$$

For the form $f=f_{m}$ we have

$$
\widehat{\mathcal{Z}}(f)=\widehat{\mathcal{Z}}_{\mathrm{Kra}}^{\mathrm{tot}}(m)=\left(\mathcal{Z}_{\mathrm{Kra}}^{\mathrm{tot}}(m), \Theta^{\mathrm{reg}}\left(f_{m}\right)\right) \in \widehat{\mathrm{Ch}}^{1}\left(\mathcal{S}_{\mathrm{Kra}}^{*}\right)
$$

where the Green function $\Theta^{\text {reg }}\left(f_{m}\right)$ for the divisor $\mathcal{Z}_{\text {Kra }}^{\text {tot }}(m)$ is constructed in [BHKRYa, §7] as a regularized theta lift. The arithmetic degree appearing 
in Theorem 4.4.1 decomposes as

$$
\begin{aligned}
{\left[\widehat{\mathcal{Z}}(f): \mathcal{Y}_{\text {big }}\right]=} & \sum_{\mathfrak{p} \subset \mathcal{O}_{k}} \log (\mathrm{N}(\mathfrak{p})) \sum_{y \in\left(\mathcal{Z}_{\text {Kra }}^{\text {tot }}(m) \cap \mathcal{Y}_{\text {big }}\right)\left(\mathbb{F}_{\mathfrak{p}}^{\text {alg }}\right)} \frac{\operatorname{length}\left(\mathcal{O}_{y}\right)}{|\operatorname{Aut}(y)|} \\
& +\sum_{y \in \mathcal{Y}_{\text {big }}(\mathbb{C})} \frac{\Theta^{\mathrm{reg}}\left(f_{m}\right)(y)}{|\operatorname{Aut}(y)|}
\end{aligned}
$$

where $\mathbb{F}_{\mathfrak{p}}=\mathcal{O}_{\boldsymbol{k}} / \mathfrak{p}$, and $\mathcal{O}_{y}$ is the étale local ring of

$$
\mathcal{Z}_{\text {Kra }}^{\text {tot }}(m) \cap \mathcal{Y}_{\text {big }} \stackrel{\text { def }}{=} \mathcal{Z}_{\text {Kra }}^{\text {tot }}(m) \times_{\mathcal{S}_{\text {Kra }}^{*}} \mathcal{Y}_{\text {big }}
$$

at $y$. The final summation is over all complex points of $\mathcal{Y}_{\text {big }}$, viewed as an $\mathcal{O}_{\boldsymbol{k}}$-stack. We will see that the terms on the right hand side of (4.4.1) are intimately related to the Eisenstein series coefficients $a_{F}(\alpha)$ of 4.3 .

We first study the structure of the stack-theoretic intersection (4.4.2). Suppose $S$ is a connected $\mathcal{O}_{\Phi^{-}}$-scheme, and

$$
\left(A_{0}, A\right) \in\left(\mathcal{M}_{(1,0)} \times \mathcal{O}_{k} \mathcal{C} \mathcal{M}_{\Phi}\right)(S)
$$

is an $S$-point. The $\mathcal{O}_{\boldsymbol{k}}$-module $\operatorname{Hom}_{\mathcal{O}_{\boldsymbol{k}}}\left(A_{0}, A\right)$ carries an $\mathcal{O}_{\boldsymbol{k}}$-hermitian form $\langle-,-\rangle$ defined by [BHKRYa, (2.5.1)]. The construction of this hermitian form only uses the underlying point of $\mathcal{S}_{\mathrm{Kra}}$, and not the action $\mathcal{O}_{E} \rightarrow$ $\operatorname{End}_{\mathcal{O}_{k}}(A)$. As in [How15, §3.2], the extra action of $\mathcal{O}_{E}$ makes $\operatorname{Hom}_{\mathcal{O}_{k}}\left(A_{0}, A\right)$ into a projective $\mathcal{O}_{E}$-module, and there is a totally positive definite $E$ hermitian form $\langle-,-\rangle_{\text {big }}$ on

$$
\mathscr{V}\left(A_{0}, A\right)=\operatorname{Hom}_{\mathcal{O}_{\boldsymbol{k}}}\left(A_{0}, A\right) \otimes_{\mathbb{Z}} \mathbb{Q}
$$

characterized by the relation

$$
\left\langle x_{1}, x_{2}\right\rangle=\operatorname{Tr}_{E / \boldsymbol{k}}\left\langle x_{1}, x_{2}\right\rangle_{\text {big }} .
$$

for all $x_{1}, x_{2} \in \operatorname{Hom}_{\mathcal{O}_{k}}\left(A_{0}, A\right)$.

Fix an $\alpha \in F_{+}$. Recalling that

$$
\mathcal{Y}_{\text {big }} \subset \mathcal{M}_{(1,0)} \times \mathcal{O}_{k} \mathcal{C} \mathcal{M}_{\Phi}
$$

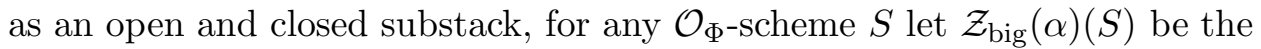
groupoid of triples $\left(A_{0}, A, x\right)$, in which

- $\left(A_{0}, A\right) \in \mathcal{Y}_{\text {big }}(S)$,

- $x \in \operatorname{Hom}_{\mathcal{O}_{k}}\left(A_{0}, A\right)$ satisfies $\langle x, x\rangle_{\mathrm{big}}=\alpha$.

This functor is represented by an $\mathcal{O}_{\Phi}$-stack $\mathcal{Z}_{\text {big }}(\alpha)$, and the evident forgetful morphism

$$
\mathcal{Z}_{\text {big }}(\alpha) \rightarrow \mathcal{Y}_{\text {big }}
$$

is finite and unramified.

This construction is entirely analogous to the construction of the special divisors $\mathcal{Z}_{\text {Kra }}^{\text {tot }}(m) \rightarrow \mathcal{S}_{\text {Kra }}$ of [BHKRYa]. In fact, directly from the definitions, if $S$ is an $\mathcal{O}_{\Phi}$-scheme an $S$-point

$$
\left(A_{0}, A, x\right) \in\left(\mathcal{Z}_{\text {Krat }}^{\text {tot }}(m) \cap \mathcal{Y}_{\text {big }}\right)(S)
$$


consists of a pair $\left(A_{0}, A\right) \in \mathcal{Y}_{\text {big }}(S)$ and an $x \in \operatorname{Hom}_{\mathcal{O}_{k}}\left(A_{0}, A\right)$ satisfying $m=\langle x, x\rangle$. From this it is clear that there is an isomorphism

$$
\mathcal{Z}_{\text {Kra }}^{\text {tot }}(m) \cap \mathcal{Y}_{\text {big }} \cong \bigsqcup_{\substack{\alpha \in F_{+} \\ \operatorname{Tr}_{F / \mathbb{Q}}(\alpha)=m}} \mathcal{Z}_{\text {big }}(\alpha),
$$

defined by sending the triple $\left(A_{0}, A, x\right)$ to the same triple, but now viewed as an $S$-point of the stack $\mathcal{Z}_{\text {big }}(\alpha)$ determined by $\alpha=\langle x, x\rangle_{\text {big }}$.

Proposition 4.4.2. For each $\alpha \in F_{+}$the stack $\mathcal{Z}_{\text {big }}(\alpha)$ is either empty, or has dimension 0 and is supported at a single prime of $\mathcal{O}_{\Phi}$. Moreover,

(1) If $|\operatorname{Diff}(\mathscr{C}, \alpha)|>1$ then $\mathcal{Z}_{\text {big }}(\alpha)=\varnothing$.

(2) Suppose that $\operatorname{Diff}(\mathscr{C}, \alpha)=\{\mathfrak{p}\}$ for a single prime $\mathfrak{p} \subset \mathcal{O}_{F}$, let $\mathfrak{q} \subset \mathcal{O}_{E}$ be the unique prime above it, and denote by $\mathfrak{q}_{\Phi} \subset \mathcal{O}_{\Phi}$ the corresponding prime under the isomorphism $\varphi^{\mathrm{sp}}: E \cong E_{\Phi}$. Then $\mathcal{Z}_{\mathrm{big}}(\alpha)$ is supported at the prime $\mathfrak{q}_{\Phi}$, and satisfies

$$
\sum_{y \in \mathcal{Z}_{\mathrm{big}}(\alpha)\left(\mathbb{F}_{\mathfrak{q}_{\Phi}}^{\text {alg }}\right)} \frac{1}{|\operatorname{Aut}(y)|}=\frac{h_{\boldsymbol{k}}}{w_{\boldsymbol{k}}} \cdot \rho\left(\alpha \mathfrak{d}_{F} \mathfrak{p}^{-\epsilon_{\mathfrak{p}}}\right),
$$

where $\mathbb{F}_{\mathfrak{q}_{\Phi}}$ is the residue field of $\mathfrak{q}_{\Phi}$, and $\epsilon_{\mathfrak{p}}$ and $\rho$ are as in Proposition 4.3.2. Moreover, the étale local rings at all geometric points

$$
y \in \mathcal{Z}_{\text {big }}(\alpha)\left(\mathbb{F}_{\mathfrak{q}_{\Phi}}^{\text {alg }}\right)
$$

have the same length

$$
\operatorname{length}\left(\mathcal{O}_{y}\right)=\operatorname{ord}_{\mathfrak{p}}\left(\alpha \mathfrak{p d} \mathfrak{d}_{F}\right) \cdot \begin{cases}1 / 2 & \text { if } E_{\mathfrak{q}} / F_{\mathfrak{p}} \text { is unramified } \\ 1 & \text { otherwise. }\end{cases}
$$

Proof. This is essentially contained in [How12, §3]. In that work we studied the $\mathcal{O}_{\Phi}$-stack $\mathcal{Z}_{\Phi}(\alpha)$ classifying triples $\left(A_{0}, A, x\right)$ exactly as in the definition of $\mathcal{Z}_{\text {big }}(\alpha)$, except we allowed the pair $\left(A_{0}, A\right)$ to be any point of $\mathcal{M}_{(1,0)} \times \mathcal{O}_{\boldsymbol{k}}$ $\mathcal{C M}_{\Phi}$ rather than a point of the substack (4.4.4). Thus we have a cartesian diagram

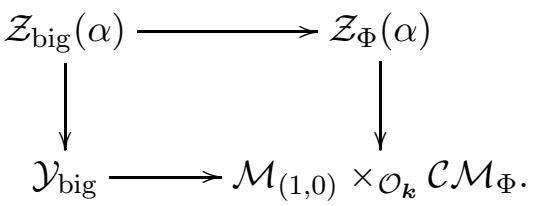

As the bottom horizontal arrow is an open and closed immersion, so is the top horizontal arrow. In other words, our $\mathcal{Z}_{\text {big }}(\alpha)$ is a union of connected components of the stack $\mathcal{Z}_{\Phi}(\alpha)$ of [How12].

Lemma 4.4.3. Each $\mathcal{Z}_{\Phi}(\alpha)$ has dimension 0 . If $y$ is a geometric point of $\mathcal{Z}_{\Phi}(\alpha)$ corresponding to a triple $\left(A_{0}, A, x\right)$ over $k(y)$, then $k(y)$ has nonzero characteristic, $A_{0}$ and $A$ are supersingular, and the E-hermitian space (4.4.3) 
has dimension one. Moreover, if $\mathfrak{p} \subset \mathcal{O}_{F}$ denotes the image of $y$ under the composition

$$
\mathcal{Z}_{\Phi}(\alpha) \rightarrow \operatorname{Spec}\left(\mathcal{O}_{\Phi}\right) \cong \operatorname{Spec}\left(\mathcal{O}_{E}\right) \rightarrow \operatorname{Spec}\left(\mathcal{O}_{F}\right)
$$

(the isomorphism is $\varphi^{\mathrm{sp}}: E \cong E_{\Phi}$ ), then $\mathfrak{p}$ is nonsplit in $E$, and the following are equivalent:

- The geometric point y factors through the open and closed substack

$$
\mathcal{Z}_{\text {big }}(\alpha) \subset \mathcal{Z}_{\Phi}(\alpha)
$$

- The E-hermitian space 4.4.3) is isomorphic to $\mathscr{V}$ everywhere locally except at $\mathfrak{p}$ and $\left.\varphi^{\mathrm{sp}}\right|_{F}$.

Proof. This is an easy consequence of [How12, Proposition 3.4.5] and [How12, Proposition 3.5.2]. The only part that requires explanation is the final claim.

Fix a connected component

$$
\mathcal{B} \subset \mathcal{M}_{(1,0)} \times{ }_{\mathcal{O}_{k}} \mathcal{C} \mathcal{M}_{\Phi}
$$

As in [How12, §3.4], for each complex point $y=\left(A_{0}, A\right) \in \mathcal{B}(\mathbb{C})$ one can construct from the Betti realizations of $A_{0}$ and $A$ an $E$-hermitian space

$$
\mathscr{V}(\mathcal{B})=\operatorname{Hom}_{\boldsymbol{k}}\left(H_{1}\left(A_{0}(\mathbb{C}), \mathbb{Q}\right), H_{1}(A(\mathbb{C}), \mathbb{Q})\right)
$$

of dimension 1. This hermitian space has signature $(0,1)$ at $\left.\varphi^{\mathrm{sp}}\right|_{F}$, and signature $(1,0)$ at all other archimedean places of $F$. Moreover, as in Remark 4.2.3, this hermitian space depends only on the connected component $\mathcal{B}$, and not on the particular complex point $y$. The open and closed substack

$$
\mathcal{Y}_{\text {big }} \subset \mathcal{M}_{(1,0)} \times \mathcal{O}_{k} \mathcal{C} \mathcal{M}_{\Phi}
$$

can be characterized as the union of all components $\mathcal{B}$ for which $\mathscr{V}(\mathcal{B}) \cong \mathscr{V}$.

So, suppose we have a geometric point $y=\left(A_{0}, A, x\right)$ of $\mathcal{Z}_{\Phi}(\alpha)$, and denote by

$$
\mathcal{B} \subset \mathcal{M}_{(1,0)} \times{ }_{\mathcal{O}_{k}} \mathcal{C} \mathcal{M}_{\Phi}
$$

the connected component containing the underlying point $y=\left(A_{0}, A\right)$. The content of [How12, Proposition 3.4.5] is that the hermitian space (4.4.3) is isomorphic to $\mathscr{V}(\mathcal{B})$ everywhere locally except at $\mathfrak{p}$ and $\left.\varphi^{\mathrm{sp}}\right|_{F}$. From this we deduce the equivalence of the following statements:

- The geometric point $y \rightarrow \mathcal{Z}_{\Phi}(\alpha)$ factors through $\mathcal{Z}_{\text {big }}(\alpha)$.

- The underlying point $y \rightarrow \mathcal{M}_{(1,0)} \times \mathcal{O}_{k} \mathcal{C M}_{\Phi}$ factors through $\mathcal{Y}_{\text {big }}$.

- The hermitian spaces $\mathscr{V}(\mathcal{B})$ and $\mathscr{V}$ are isomorphic.

- The $E$-hermitian space (4.4.3) is isomorphic to $\mathscr{V}$ everywhere locally except at $\mathfrak{p}$ and $\left.\varphi^{\mathrm{sp}}\right|_{F}$.

Now suppose that $\mathcal{Z}_{\mathrm{big}}(\alpha)$ is nonempty. If we fix a geometric point $y=\left(A_{0}, A, x\right)$ as above, the vector $x \in \operatorname{Hom}_{\mathcal{O}_{k}}\left(A_{0}, A\right)$ satisfies $\langle x, x\rangle_{\text {big }}=\alpha$, and hence (4.4.3) represents $\alpha$. The above lemma now implies that $\mathscr{V}$ represents $\alpha$ everywhere locally except at $\mathfrak{p}$ and $\left.\varphi^{\mathrm{sp}}\right|_{F}$, where $\mathfrak{p}$ is the image of 
$y$ under (4.4.6). From this it follows first $\operatorname{Diff}(\mathscr{C}, \alpha)=\{\mathfrak{p}\}$, and then that all geometric points of $\mathcal{Z}_{\text {big }}(\alpha)$ have the same image under (4.4.6), and lie above the same prime $\mathfrak{q}_{\Phi} \subset \mathcal{O}_{\Phi}$ characterized as in the statement of Proposition 4.4.2. In particular, if $|\operatorname{Diff}(\mathscr{C}, \alpha)|>1$ then $\mathcal{Z}_{\text {big }}(\alpha)=\varnothing$.

It remains to prove part (2) of the proposition. For this we need the following lemma.

Lemma 4.4.4. Assume that $\operatorname{Diff}(\mathscr{C}, \alpha)=\{\mathfrak{p}\}$ for some prime $\mathfrak{p} \subset \mathcal{O}_{F}$, and let $\mathfrak{q} \subset \mathcal{O}_{E}$ be the unique prime above it. The open and closed substack $\mathcal{Z}_{\text {big }}(\alpha) \subset \mathcal{Z}_{\Phi}(\alpha)$ is equal to the union of all connected components of $\mathcal{Z}_{\Phi}(\alpha)$ that are supported at the prime $\mathfrak{q}_{\Phi}$.

Proof. We have already seen that every geometric point of $\mathcal{Z}_{\text {big }}(\alpha)$ lies above the prime $\mathfrak{q}_{\Phi}$, and so it suffices to prove that every geometric point of $\mathcal{Z}_{\Phi}(\alpha)$ lying above the prime $\mathfrak{q}_{\Phi}$ factors through $\mathcal{Z}_{\text {big }}(\alpha)$. Let $y \rightarrow \mathcal{Z}_{\Phi}(\alpha)$ be such a point.

If $y$ corresponds to the triple $\left(A_{0}, A, x\right)$, then $x \in \operatorname{Hom}_{\mathcal{O}_{k}}\left(A_{0}, A\right)$ satisfies $\langle x, x\rangle_{\text {big }}=\alpha$, and hence (4.4.3) represents $\alpha$. But the assumption that $\operatorname{Diff}(\mathscr{C}, \alpha)=\{\mathfrak{p}\}$ implies that $\mathscr{V}$ represents $\alpha$ everywhere locally except at $\mathfrak{p}$ and $\left.\varphi^{\mathrm{sp}}\right|_{F}$, and it follows from this that $\mathscr{V}$ and (4.4.3) are isomorphic locally everywhere except at $\mathfrak{p}$ and $\left.\varphi^{\mathrm{sp}}\right|_{F}$. By the previous lemma, this implies that $y$ factors through $\mathcal{Z}_{\text {big }}(\alpha)$.

With this last lemma in hand, all parts of (2) follow from the corresponding statements for $\mathcal{Z}_{\Phi}(\alpha)$ proved in [How12, Theorem 3.5.3] and [How12, Theorem 3.6.2].

Proposition 4.4.5. For every $\alpha \in F_{+}$we have

$$
\sum_{\mathfrak{p} \subset \mathcal{O}_{k}} \frac{n \cdot \log (\mathrm{N}(\mathfrak{p}))}{\operatorname{deg}_{\mathbb{C}}\left(\mathcal{Y}_{\text {big }}\right)} \sum_{y \in \mathcal{Z}_{\text {big }}(\alpha)\left(\mathbb{F}_{\mathfrak{p}}^{\text {alg }}\right)} \frac{\operatorname{length}\left(\mathcal{O}_{y}\right)}{|\operatorname{Aut}(y)|}=-\frac{a_{F}(\alpha, 0)}{\Lambda\left(0, \chi_{E}\right)},
$$

where the inner sum is over all $\mathbb{F}_{\mathfrak{p}}^{\text {alg }}$-points of $\mathcal{Z}_{\mathrm{big}}(\alpha)$, viewed as an $\mathcal{O}_{\boldsymbol{k}}$-stack.

Proof. Combining Propositions 4.2.8, 4.3.2, and 4.4.2 shows that

$$
\sum_{\mathfrak{q}_{\Phi} \subset \mathcal{O}_{\Phi}} \frac{n \cdot \log \left(\mathrm{N}\left(\mathfrak{q}_{\Phi}\right)\right)}{\operatorname{deg}_{\mathbb{C}}\left(\mathcal{Y}_{\text {big }}\right)} \sum_{y \in \mathcal{Z}_{\text {big }}(\alpha)\left(\mathbb{F}_{\mathfrak{q}_{\Phi}}^{\text {alg }}\right)} \frac{\operatorname{length}\left(\mathcal{O}_{y}\right)}{|\operatorname{Aut}(y)|}=-\frac{a_{F}(\alpha, 0)}{\Lambda\left(0, \chi_{E}\right)},
$$

where the inner sum is over all $\mathbb{F}_{\mathfrak{q}_{\Phi}}^{\text {alg }}$ points of $\mathcal{Z}_{\text {big }}(\alpha)$, viewed as an $\mathcal{O}_{\Phi}$-stack. The claim follows by collecting together all primes $\mathfrak{q}_{\Phi} \subset \mathcal{O}_{\Phi}$ lying above a common prime $\mathfrak{p} \subset \mathcal{O}_{\boldsymbol{k}}$. 
Proposition 4.4.6. The regularized theta lift $\Theta^{\mathrm{reg}}\left(f_{m}\right)$ satisfies

$$
\begin{aligned}
& \frac{n}{\operatorname{deg}_{\mathbb{C}}\left(\mathcal{Y}_{\text {big }}\right)} \sum_{y \in \mathcal{Y}_{\text {big }}(\mathbb{C})} \frac{\Theta^{\text {reg }}\left(f_{m}\right)(y)}{|\operatorname{Aut}(y)|} \\
= & -\left.\frac{d}{d s}\left\langle E(s), \xi\left(f_{m}\right)\right\rangle_{\text {Pet }}\right|_{s=0}+\sum_{\substack{\alpha \in F_{+} \\
\operatorname{Tr}_{F / \mathbb{Q}}(\alpha)=m}} \frac{a_{F}(\alpha, 0)}{\Lambda\left(0, \chi_{E}\right)}-2 c_{f_{m}}^{+}(0,0) \cdot \frac{\Lambda^{\prime}\left(0, \chi_{E}\right)}{\Lambda\left(0, \chi_{E}\right)} .
\end{aligned}
$$

Proof. This is a special case of the main result of BKY12. This requires some explanation, as that work deals with cycles on Shimura varieties of type GSpin, rather than the unitary Shimura varieties under current consideration.

Recall that we have an $F$-quadratic space $(\mathscr{V}, \mathscr{Q})$ of rank two, and a $\mathbb{Q}$-quadratic space $(V, Q)$ whose underlying $\mathbb{Q}$-vector space

$$
V=\operatorname{Hom}_{\boldsymbol{k}}\left(W_{0}, W\right)
$$

is equal to $\mathscr{V}$, and whose quadratic form is (4.3.1). As in [BKY12, §2] or [AGHMP18, §5.3] this data determines a commutative diagram

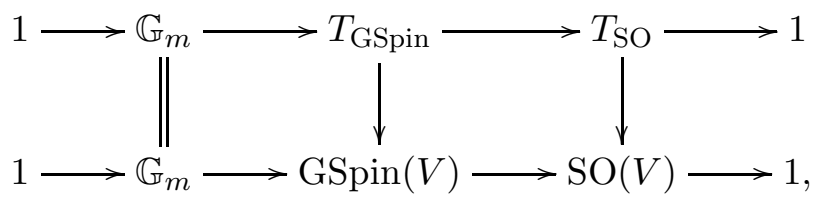

with exact rows, of algebraic groups over $\mathbb{Q}$. The torus $T_{\mathrm{SO}}=\operatorname{Res}_{F / \mathbb{Q}} \mathrm{SO}(\mathscr{V})$ has $\mathbb{Q}$-points

$$
T_{\mathrm{SO}}(\mathbb{Q})=\left\{y \in E^{\times}: y \bar{y}=1\right\},
$$

while the torus $T_{\mathrm{GSpin}}$ has $\mathbb{Q}$-points

$$
T_{\mathrm{GSpin}}(\mathbb{Q})=E^{\times} / \operatorname{ker}\left(\text { Norm }: F^{\times} \rightarrow \mathbb{Q}^{\times}\right) .
$$

The map $T_{\mathrm{GSpin}} \rightarrow T_{\mathrm{SO}}$ is $x \mapsto x / \bar{x}$. To these groups one can associate morphisms of Shimura data

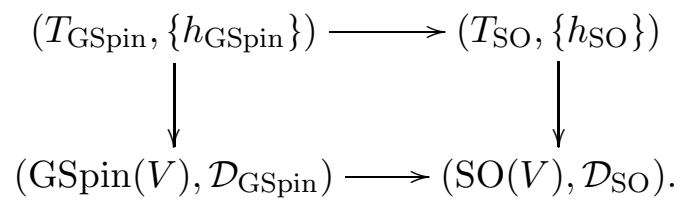

In the top row both data have reflex field $E_{\Phi}$. In the bottom row both data have reflex field $\mathbb{Q}$.

Let $K_{\mathrm{SO}} \subset \mathrm{SO}(V)\left(\mathbb{A}_{f}\right)$ be any compact open subgroup that stabilizes the lattice $L \subset V$, and fix any compact open subgroup $K_{\mathrm{GSpin}} \subset \operatorname{GSpin}(V)\left(\mathbb{A}_{f}\right)$ contained in the preimage of $K_{\mathrm{SO}}$. The Shimura data in the bottom row, along with these compact open subgroups, determine Shimura varieties $M_{\mathrm{GSpin}} \rightarrow M_{\mathrm{SO}}$. These are $\mathbb{Q}$-stacks of dimension $2 n-2$. 
The Shimura data in the top row, along with the compact open subgroups $K_{\mathrm{GSpin}} \cap T_{\mathrm{GSpin}}\left(\mathbb{A}_{f}\right)$ and $K_{\mathrm{SO}} \cap T_{\mathrm{SO}}\left(\mathbb{A}_{f}\right)$, determine Shimura vari-

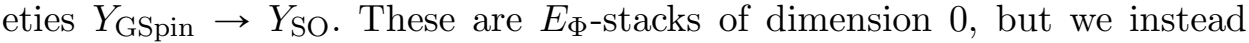
view them as stacks over $\operatorname{Spec}(\mathbb{Q})$, so that there is a commutative diagram

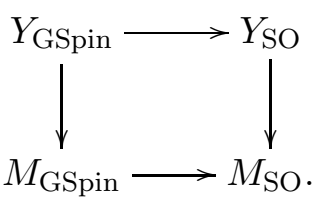

Assume that the compact open subgroup $K_{\mathrm{SO}}$ acts trivially on the quotient $L^{\prime} / L$. For every form $f \in H_{2-n}\left(\bar{\omega}_{L}\right)$, one can find in [BKY12, Theorem 3.2] the construction of a divisor $Z_{\mathrm{GSpin}}(f)$ on $M_{\mathrm{GSpin}}$, along with a Green function $\Theta_{\mathrm{GSpin}}^{\mathrm{reg}}(f)$ for that divisor, constructed as a regularized theta lift. Up to change of notation, [BKY12, Theorem 1.1] asserts that

$$
\begin{aligned}
\frac{n}{\operatorname{deg}_{\mathbb{C}}\left(Y_{\mathrm{GSpin}}\right)} \sum_{y \in Y_{\mathrm{GSpin}}(\mathbb{C})} \frac{\Theta_{\mathrm{GSpin}}^{\mathrm{reg}}(f, y)}{|\operatorname{Aut}(y)|}= & -\left.\frac{d}{d s}\langle E(s), \xi(f)\rangle_{\mathrm{Pet}}\right|_{s=0} \\
& +\sum_{\substack{m \geqslant 0 \\
\mu \in L^{\prime} / L}} \frac{a(m, \mu) c_{f}^{+}(-m, \mu)}{\Lambda\left(0, \chi_{E}\right)}
\end{aligned}
$$

where the coefficients $a(m) \in S_{L}$ are defined by

$$
a(m)=\sum_{\substack{\alpha \in F_{+} \\ \operatorname{Tr}_{F / \mathbb{Q}}(\alpha)=m}} a_{F}(\alpha)
$$

if $m>0$, and by $a(0)=a_{F}(0)$.

It is not difficult to see, directly from the constructions, that both the divisor $Z_{\mathrm{GSpin}}(f)$ and the Green function $\Theta_{\mathrm{GSpin}}^{\mathrm{reg}}(f)$ descend to the quotient $M_{\mathrm{SO}}$. If we call these descents $Z_{\mathrm{SO}}(f)$ and $\Theta_{\mathrm{SO}}^{\mathrm{reg}}(f)$, it is a formal consequence of the commutativity of (4.4.7) that the equality (4.4.8) continues to hold if all subscripts GSpin are replaced by SO.

Moreover, suppose that our form $f \in H_{2-n}\left(\bar{\omega}_{L}\right)$ is invariant under the action of the finite group $\Delta$ of 92.4 , as is true for the form $f_{m}$ of (2.5.1). In this case one can see, directly from the definitions, that the divisor $Z_{\mathrm{SO}}(f)$ and the Green function $\Theta_{\mathrm{SO}}^{\mathrm{reg}}(f)$ descend to the orthogonal Shimura variety determined by the maximal compact open subgroup

$$
K_{\mathrm{SO}}=\left\{g \in \mathrm{SO}(V)\left(\mathbb{A}_{f}\right): g L=L\right\}
$$

From now on we fix this choice of $K_{\mathrm{SO}}$. 
Specializing (4.4.8) to the form $f=f_{m}$, and using the formula for $a(0)=$ $a_{F}(0)$ found in Proposition 4.3.3, we obtain

$$
\begin{aligned}
\frac{n}{\operatorname{deg}_{\mathbb{C}}\left(Y_{\mathrm{SO}}\right)} \sum_{y \in Y_{\mathrm{SO}}(\mathbb{C})} \frac{\Theta_{\mathrm{SO}}^{\mathrm{reg}}\left(f_{m}\right)(y)}{|\operatorname{Aut}(y)|}= & -\left.\frac{d}{d s}\left\langle E(s), \xi\left(f_{m}\right)\right\rangle_{\mathrm{Pet}}\right|_{s=0} \\
& +\frac{a(m, 0)}{\Lambda\left(0, \chi_{E}\right)}-2 c_{f_{m}}^{+}(0,0) \cdot \frac{\Lambda^{\prime}\left(0, \chi_{E}\right)}{\Lambda\left(0, \chi_{E}\right)} .
\end{aligned}
$$

As in BHKRYa, §2.1], our group $G \subset \mathrm{GU}\left(W_{0}\right) \times \mathrm{GU}(W)$ acts on $V$ in a natural way, defining a homomorphism $G \rightarrow \mathrm{SO}(V)$. On the other hand, Remark 4.1.1 shows that $T_{\text {big }} \cong \operatorname{Res}_{\boldsymbol{k} / \mathbb{Q}} \mathbb{G}_{m} \times T_{\mathrm{SO}}$, and projection to the second factor defines a morphism $T_{\mathrm{big}} \rightarrow T_{\mathrm{SO}}$. We obtain morphisms of Shimura data

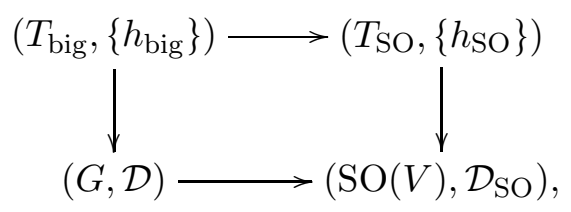

which induce morphisms of $\boldsymbol{k}$-stacks

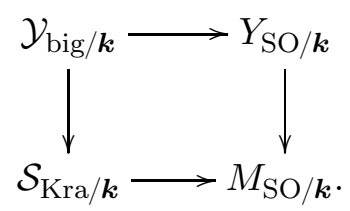

The Green function $\Theta^{\mathrm{reg}}\left(f_{m}\right)$ on $\mathcal{S}_{\mathrm{Kra} / \boldsymbol{k}}$ defined in [BHKRYa, $\S 7.2$ ] is simply the pullback of the Green function $\Theta_{\mathrm{SO}}^{\mathrm{reg}}\left(f_{m}\right)$ via the bottom horizontal arrow. It follows easily that

$$
\frac{n}{\operatorname{deg}_{\mathbb{C}}\left(Y_{\mathrm{SO}}\right)} \sum_{y \in Y_{\mathrm{SO}}(\mathbb{C})} \frac{\Theta_{\mathrm{SO}}^{\mathrm{reg}}\left(f_{m}\right)(y)}{|\operatorname{Aut}(y)|}=\frac{n}{\operatorname{deg}_{\mathbb{C}}\left(\mathcal{Y}_{\text {big }}\right)} \sum_{y \in \mathcal{Y}_{\text {big }}(\mathbb{C})} \frac{\Theta^{\mathrm{reg}}\left(f_{m}\right)(y)}{|\operatorname{Aut}(y)|},
$$

and comparison with (4.4.9) completes the proof of Proposition 4.4.6.

Proof of Theorem 4.4.1. Combining the decomposition (4.4.5) with Proposition 4.4.5 shows that

$$
\sum_{\mathfrak{p} \subset \mathcal{O}_{k}} \frac{n \log (\mathrm{N}(\mathfrak{p}))}{\operatorname{deg}_{\mathbb{C}}\left(\mathcal{Y}_{\text {big }}\right)} \sum_{y \in\left(\mathcal{Z}_{\text {Kra }}^{\text {tot }}(m) \cap \mathcal{Y}_{\text {big }}\right)\left(\mathbb{F}_{\mathfrak{p}}^{\text {alg }}\right)} \frac{\operatorname{length}\left(\mathcal{O}_{y}\right)}{|\operatorname{Aut}(y)|}=\sum_{\substack{\alpha \in F_{+} \\ \operatorname{Tr}_{F / \mathbb{Q}}(\alpha)=m}} \frac{-a_{F}(\alpha, 0)}{\Lambda\left(0, \chi_{E}\right)} .
$$

Plugging this formula and the archimedean calculation of Proposition 4.4.6 into (4.4.1) leaves

$$
\frac{n \cdot\left[\hat{\mathcal{Z}}\left(f_{m}\right): \mathcal{Y}_{\mathrm{big}}\right]}{\operatorname{deg}_{\mathbb{C}}\left(\mathcal{Y}_{\mathrm{big}}\right)}=-2 c_{f_{m}}^{+}(0,0) \cdot \frac{\Lambda^{\prime}\left(0, \chi_{E}\right)}{\Lambda\left(0, \chi_{E}\right)}-\left.\frac{d}{d s}\left\langle E(s), \xi\left(f_{m}\right)\right\rangle_{\mathrm{Pet}}\right|_{s=0},
$$

as desired. 
4.5. The proof of Theorem $\mathbf{B}$. We now use Theorem 4.4.1 to prove a special case of Theorem $\mathrm{D}$, and then prove Theorem $\mathrm{B}$, We assume $n \geqslant 3$.

Recall the differential operator

$$
\xi: H_{2-n}\left(\omega_{L}\right) \rightarrow S_{n}\left(\bar{\omega}_{L}\right)
$$

of 2.4 . Its kernel is the subspace

$$
M_{2-n}^{!}\left(\omega_{L}\right) \subset H_{2-n}\left(\omega_{L}\right)
$$

of weakly holomorphic forms.

Lemma 4.5.1. In the notation of 92.4 , there exists a $\Delta$-invariant form $f \in M_{2-n}^{!}\left(\omega_{L}\right)$ such that $c_{f}^{+}(0,0) \neq 0$, and

$$
\widehat{\mathcal{Z}}(f)+c_{f}^{+}(0,0) \cdot \hat{\mathcal{Z}}_{\mathrm{Kra}}^{\mathrm{tot}}(0)=0 .
$$

Proof. Denote by

$$
S_{2-n}^{!, \infty}\left(\Gamma_{0}(D), \chi_{\boldsymbol{k}}^{n}\right) \subset M_{2-n}^{!}\left(\Gamma_{0}(D), \chi_{\boldsymbol{k}}^{n}\right)
$$

the subspace of forms that vanish at all cusps other than $\infty$, and choose any form

$$
f_{0}(\tau)=\sum_{\substack{m \in \mathbb{Z} \\ m \gg-\infty}} c_{0}(m) \cdot q^{m} \in S_{2-n}^{!, \infty}\left(\Gamma_{0}(D), \chi_{\boldsymbol{k}}^{n}\right)
$$

such that $c_{0}(0) \neq 0$. The existence of such a form can be proved as in [BBGK07, Lemma 4.11]. As in (2.3.2) there is an induced form

$$
f(\tau)=\sum_{\gamma \in \Gamma_{0}(D) \backslash \mathrm{SL}_{2}(\mathbb{Z})}\left(\left.f_{0}\right|_{2-n} \gamma\right)(\tau) \cdot \omega_{L}\left(\gamma^{-1}\right) \phi_{0} \in M_{2-n}^{!}\left(\omega_{L}\right)^{\Delta},
$$

which we claim has the desired properties.

Indeed, the proof of Proposition 2.5.1 shows that $c_{f}^{+}(0,0)=c_{0}(0)$, and that $f=\sum_{m>0} c_{0}(-m) f_{m}$. In particular,

$$
\widehat{\mathcal{Z}}(f)=\sum_{m>0} c_{0}(-m) \cdot \widehat{\mathcal{Z}}_{\mathrm{Kra}}^{\mathrm{tot}}(m) \in \widehat{\mathrm{Ch}}_{\mathbb{C}}^{1}\left(\mathcal{S}_{\mathrm{Kra}}^{*}\right) .
$$

Given any modular form

$$
g(\tau)=\sum_{m \geqslant 0} d(m) \cdot q^{m} \in M_{n}\left(D, \chi_{\boldsymbol{k}}^{n}\right),
$$

summing the residues of the meromorphic form $f_{0}(\tau) g(\tau) d \tau$ on $X_{0}(D)(\mathbb{C})$ shows that

$$
\sum_{m \geqslant 0} c_{0}(-m) \cdot d(m)=0 .
$$

Thus the modularity of the generating series (1.1.2) implies the second equality in

$$
\widehat{\mathcal{Z}}(f)+c_{0}(0) \cdot \hat{\mathcal{Z}}_{\mathrm{Kra}}^{\mathrm{tot}}(0)=\sum_{m \geqslant 0} c_{0}(-m) \cdot \widehat{\mathcal{Z}}_{\mathrm{Kra}}^{\mathrm{tot}}(m)=0 .
$$


We can now prove Theorem $\mathrm{D}$ under some additional hypotheses. These hypotheses will be removed in $\$ 5$.

Theorem 4.5.2. If the discriminants of $\boldsymbol{k} / \mathbb{Q}$ and $F / \mathbb{Q}$ are odd and relatively prime, then

$$
\left[\widehat{\boldsymbol{\omega}}: \mathcal{Y}_{\text {big }}\right]=\frac{-2}{n} \cdot \operatorname{deg}_{\mathbb{C}}\left(\mathcal{Y}_{\text {big }}\right) \cdot \frac{\Lambda^{\prime}\left(0, \chi_{E}\right)}{\Lambda\left(0, \chi_{E}\right)}
$$

Proof. If we choose $f$ as in Lemma 4.5.1 then $\xi(f)=0$, and so Theorem 4.4 .1 simplifies to

$$
-n c_{f}^{+}(0,0) \cdot \frac{\left[\hat{\mathcal{Z}}_{\mathrm{Kra}}^{\mathrm{tot}}(0): \mathcal{Y}_{\mathrm{big}}\right]}{\operatorname{deg}_{\mathbb{C}}\left(\mathcal{Y}_{\mathrm{big}}\right)}+2 c_{f}^{+}(0,0) \cdot \frac{\Lambda^{\prime}\left(0, \chi_{E}\right)}{\Lambda\left(0, \chi_{E}\right)}=0 .
$$

An application of Proposition 4.2.11 completes the proof.

The following is Theorem $\mathrm{B}$ in the introduction.

Theorem 4.5.3. Assume that the discriminants of $\boldsymbol{k} / \mathbb{Q}$ and $F / \mathbb{Q}$ are odd and relatively prime, and let $g \in S_{n}\left(\Gamma_{0}(D), \chi^{n}\right)$ and $\tilde{g} \in S_{n}\left(\bar{\omega}_{L}\right)$ be related by (2.3.2). The central derivative of the Petersson inner product (4.3.4) is related to the arithmetic theta lift (1.1.3) by

$$
\left[\hat{\theta}(g): \mathcal{Y}_{\text {big }}\right]=\left.\frac{-1}{n} \cdot \operatorname{deg}_{\mathbb{C}}\left(\mathcal{Y}_{\text {big }}\right) \cdot \frac{d}{d s}\langle E(s), \tilde{g}\rangle_{\text {Pet }}\right|_{s=0} .
$$

Proof. If we choose $f$ as in Proposition 2.5.1, then $\xi(f)=\tilde{g}$ and

$$
\left[\widehat{\theta}(g): \mathcal{Y}_{\mathrm{big}}\right]=\left[\widehat{\mathcal{Z}}(f): \mathcal{Y}_{\mathrm{big}}\right]+c_{f}^{+}(0,0) \cdot\left[\hat{\mathcal{Z}}_{\mathrm{Kra}}^{\mathrm{tot}}(0): \mathcal{Y}_{\mathrm{big}}\right]
$$

Proposition 4.2 .11 and Theorem 4.5.2 allow us to rewrite this as

$$
\begin{aligned}
{\left[\hat{\theta}(g): \mathcal{Y}_{\mathrm{big}}\right] } & =\left[\widehat{\mathcal{Z}}(f): \mathcal{Y}_{\mathrm{big}}\right]-c_{f}^{+}(0,0) \cdot\left[\widehat{\boldsymbol{\omega}}: \mathcal{Y}_{\mathrm{big}}\right] \\
& =\left[\widehat{\mathcal{Z}}(f): \mathcal{Y}_{\mathrm{big}}\right]+\frac{2}{n} \cdot c_{f}^{+}(0,0) \cdot \operatorname{deg}_{\mathbb{C}}\left(\mathcal{Y}_{\mathrm{big}}\right) \cdot \frac{\Lambda^{\prime}\left(0, \chi_{E}\right)}{\Lambda\left(0, \chi_{E}\right)},
\end{aligned}
$$

and comparison with Theorem 4.4.1 completes the proof.

\section{Faltings heights of CM ABELian varieties}

In $\$ 5$ we assume $n \geqslant 2$, and study Theorems $\mathrm{C}$ and $\mathrm{D}$ of the introduction. As in $§ 1.3$, let $F$ be a totally real field of degree $n$, set

$$
E=\boldsymbol{k} \otimes_{\mathbb{Q}} F,
$$

and let $\Phi \subset \operatorname{Hom}(E, \mathbb{C})$ be a CM type of signature $(n-1,1)$. We fix a triple $\left(\mathfrak{a}_{0}, \mathfrak{a}, i_{E}\right)$ as in 4.2 . 
5.1. Some metrized line bundles. By virtue of the inclusion (1.1.1), there is a universal pair $\left(A_{0}, A\right)$ over $\mathcal{S}_{\mathrm{Kra}}$ consisting of an elliptic curve $\pi_{0}: A_{0} \rightarrow \mathcal{S}_{\mathrm{Kra}}$ and an abelian scheme $\pi: A \rightarrow \mathcal{S}_{\mathrm{Kra}}$ of dimension $n$.

Endowing the Lie algebras of $A_{0}$ and $A$ with their Faltings (a.k.a. Hodge) metrics gives rise to metrized line bundles

$$
\operatorname{Lie}\left(A_{0}\right) \in \widehat{\operatorname{Pic}}\left(\mathcal{S}_{\mathrm{Kra}}\right), \quad \operatorname{det}(\operatorname{Lie}(A)) \in \widehat{\operatorname{Pic}}\left(\mathcal{S}_{\mathrm{Kra}}\right) .
$$

A vector $\eta$ in the fiber

$$
\operatorname{det}\left(\operatorname{Lie}\left(A_{s}\right)\right)^{-1} \cong \bigwedge^{n} \operatorname{Fil}^{1} H_{\mathrm{dR}}^{1}\left(A_{s}\right) \subset \bigwedge^{n} H_{\mathrm{dR}}^{1}\left(A_{s}\right)
$$

at a complex point $s \in \mathcal{S}_{\mathrm{Kra}}(\mathbb{C})$ has norm

$$
\|\eta\|_{s}^{2}=\left|\int_{A_{s}(\mathbb{C})} \eta \wedge \bar{\eta}\right|
$$

The metric on $\operatorname{Lie}\left(A_{0}\right)$ is defined similarly.

We now recall some notation from [BHKRYa, §1.8]. Fix a $\pi \in \mathcal{O}_{\boldsymbol{k}}$ such

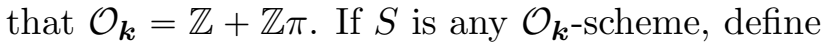

$$
\begin{aligned}
& \epsilon_{S}=\pi \otimes 1-1 \otimes i_{S}(\bar{\pi}) \in \mathcal{O}_{\boldsymbol{k}} \otimes_{\mathbb{Z}} \mathcal{O}_{S} \\
& \bar{\epsilon}_{S}=\bar{\pi} \otimes 1-1 \otimes i_{S}(\bar{\pi}) \in \mathcal{O}_{\boldsymbol{k}} \otimes_{\mathbb{Z}} \mathcal{O}_{S},
\end{aligned}
$$

where $i_{S}: \mathcal{O}_{\boldsymbol{k}} \rightarrow \mathcal{O}_{S}$ is the structure map. We usually just write $\epsilon$ and $\bar{\epsilon}$, when the scheme $S$ is clear from context.

Remark 5.1.1. If $N$ is an $\mathcal{O}_{\boldsymbol{k}} \otimes_{\mathbb{Z}} \mathcal{O}_{S}$-module then $N / \bar{\epsilon} N$ is the maximal quotient of $N$ on which $\mathcal{O}_{\boldsymbol{k}}$ acts through the structure morphism $i_{S}: \mathcal{O}_{\boldsymbol{k}} \rightarrow \mathcal{O}_{S}$, and $N / \epsilon N$ is the maximal quotient on which $\mathcal{O}_{\boldsymbol{k}}$ acts through the conjugate of the structure morphism. If $D \in \mathcal{O}_{S}^{\times}$then

$$
N=\epsilon N \oplus \bar{\epsilon} N
$$

and the summands are the maximal submodules on which $\mathcal{O}_{\boldsymbol{k}}$ acts through the structure morphism and its conjugate, respectively.

As in BHKRYa, §2.2], the relative de Rham homology $H_{1}^{\mathrm{dR}}(A)$ is a rank $2 n$ vector bundle on $\mathcal{S}_{\mathrm{Kra}}$ endowed with an action of $\mathcal{O}_{\boldsymbol{k}}$ induced from that on $A$. In fact, it is locally free of rank $n$ as an $\mathcal{O}_{\boldsymbol{k}} \otimes_{\mathbb{Z}} \mathcal{O}_{\mathcal{S}_{\mathrm{Kra}}}$-module, and

$$
\mathcal{V}=H_{1}^{\mathrm{dR}}(A) / \bar{\epsilon} H_{1}^{\mathrm{dR}}(A)
$$

is a rank $n$ vector bundle. We make $\operatorname{det}(\mathcal{V})$ into a metrized line bundle by declaring that a local section $\eta$ of its inverse

$$
\operatorname{det}(\mathcal{V})^{-1} \cong \bigwedge^{n} \epsilon H_{\mathrm{dR}}^{1}(A) \subset H_{\mathrm{dR}}^{n}(A)
$$

has norm (5.1.1) at a complex point $s \in \mathcal{S}_{\mathrm{Kra}}(\mathbb{C})$.

As the exceptional divisor Exc $\subset \mathcal{S}_{\mathrm{Kra}}$ of [BHKRYa, §2.3] is supported in characteristics dividing $D$, the line bundle $\mathcal{O}$ (Exc) is canonically trivial in the generic fiber. We endow it with the trivial metric. That is to say, the constant function 1 , viewed as a section of $\mathcal{O}(\mathrm{Exc})$, has norm $\|1\|^{2}=1$. 
Recall that the line bundle $\boldsymbol{\omega}$ of [BHKRYa, §2.4] was endowed with a metric in BHKRYa, $§ 7.2]$, defining

$$
\widehat{\omega} \in \widehat{\operatorname{Pic}}\left(\mathcal{S}_{\mathrm{Kra}}\right) \text {. }
$$

For any positive real number $c$, denote by

$$
\mathcal{O}\langle c\rangle \in \widehat{\operatorname{Pic}}\left(\mathcal{S}_{\text {Kra }}\right)
$$

the trivial bundle $\mathcal{O}_{\mathcal{S}_{\mathrm{Kra}}}$ endowed with the constant metric $\|1\|^{2}=c$.

Proposition 5.1.2. There is an isomorphism

$$
\mathcal{O}\left\langle 8 \pi^{2} e^{\gamma} D^{-1}\right\rangle^{\otimes 2} \otimes \widehat{\boldsymbol{\omega}}^{\otimes 2} \otimes \operatorname{det}(\operatorname{Lie}(A)) \otimes \operatorname{Lie}\left(A_{0}\right)^{\otimes 2} \cong \mathcal{O}(\operatorname{Exc}) \otimes \operatorname{det}(\mathcal{V})
$$

of metrized line bundles on $\mathcal{S}_{\mathrm{Kra}}$.

Proof. In [BHKRYa, §2.4] we defined a line bundle $\boldsymbol{\Omega}_{\mathrm{Kra}}$ on $\mathcal{S}_{\mathrm{Kra}}$ by

$$
\boldsymbol{\Omega}_{\mathrm{Kra}}=\operatorname{det}(\operatorname{Lie}(A))^{-1} \otimes \operatorname{Lie}\left(A_{0}\right)^{\otimes-2} \otimes \operatorname{det}(\mathcal{V}),
$$

and in [BHKRYa, Theorem 2.6.3] we constructed an isomorphism

$$
\omega^{\otimes 2} \cong \Omega_{\mathrm{Kra}} \otimes \mathcal{O}(\mathrm{Exc}) .
$$

This defines the desired isomorphism

$$
\boldsymbol{\omega}^{\otimes 2} \otimes \operatorname{det}(\operatorname{Lie}(A)) \otimes \operatorname{Lie}\left(A_{0}\right)^{\otimes 2} \cong \mathcal{O}(\operatorname{Exc}) \otimes \operatorname{det}(\mathcal{V})
$$

on underlying line bundles, and it remains to compare the metrics.

In the complex fiber this can be made more explicit. At any complex point $s \in \mathcal{S}_{\mathrm{Kra}}(\mathbb{C})$ the Hodge short exact sequence admits a canonical splitting

$$
H_{1}^{\mathrm{dR}}\left(A_{s}\right)=F^{0}\left(A_{s}\right) \oplus \operatorname{Lie}\left(A_{s}\right),
$$

where $F^{0}\left(A_{s}\right)=\mathrm{Fil}^{0} H_{1}^{\mathrm{dR}}\left(A_{s}\right)$ is the nontrivial step in the Hodge filtration. When combined with the decomposition of Remark 5.1.1 we obtain

$$
H_{1}^{\mathrm{dR}}\left(A_{s}\right)=\underbrace{\epsilon F^{0}\left(A_{s}\right)}_{1} \oplus \underbrace{\bar{\epsilon} F^{0}\left(A_{s}\right)}_{n-1} \oplus \underbrace{\epsilon \operatorname{Lie}\left(A_{s}\right)}_{n-1} \oplus \underbrace{\bar{\epsilon} \operatorname{Lie}\left(A_{s}\right)}_{1}
$$

where the subscripts indicate the dimensions as $\mathbb{C}$-vector spaces. There is a similar decomposition

$$
H_{1}^{\mathrm{dR}}\left(A_{0 s}\right)=\underbrace{\epsilon F^{0}\left(A_{0 s}\right)}_{0} \oplus \underbrace{\bar{\epsilon} F^{0}\left(A_{0 s}\right)}_{1} \oplus \underbrace{\epsilon \operatorname{Lie}\left(A_{0 s}\right)}_{1} \oplus \underbrace{\bar{\epsilon} \operatorname{Lie}\left(A_{0 s}\right)}_{0}
$$

Denote by

$$
\psi: H_{1}^{\mathrm{dR}}\left(A_{s}\right) \times H_{1}^{\mathrm{dR}}\left(A_{s}\right) \rightarrow \mathbb{C}
$$

the alternating pairing determined by the principal polarization on $A_{s}$. The two direct summands

$$
\epsilon F^{0}\left(A_{s}\right) \oplus \bar{\epsilon} \operatorname{Lie}\left(A_{s}\right) \subset H_{1}^{\mathrm{dR}}\left(A_{s}\right)
$$


are interchanged by complex conjugation. We endow both $\epsilon F^{0}\left(A_{s}\right)$ and $\bar{\epsilon} \operatorname{Lie}\left(A_{s}\right)$ with the metric

$$
\|b\|_{s}^{2}=\left|\frac{\psi(b, \bar{b})}{2 \pi i}\right|
$$

so that the pairing

$$
\psi: \epsilon F^{0}\left(A_{s}\right) \otimes \bar{\epsilon} \operatorname{Lie}\left(A_{s}\right) \rightarrow \mathcal{O}\left\langle 4 \pi^{2}\right\rangle_{s}^{-1}
$$

is an isometry.

For $a, b \in \bar{\epsilon} \operatorname{Lie}\left(A_{s}\right)$, define $p_{a \otimes b}: \epsilon F^{0}\left(A_{s}\right) \rightarrow \bar{\epsilon} \operatorname{Lie}\left(A_{s}\right)$ by

$$
p_{a \otimes b}(e)=\psi(\bar{\epsilon} a, e) \cdot \bar{\epsilon} b=-D \psi(a, e) \cdot b .
$$

The factor of $-D$ comes from the observation that $\bar{\epsilon}$ acts on $\bar{\epsilon} \operatorname{Lie}\left(A_{s}\right)$ as $\pm \sqrt{-D}$, where the sign depends on the choice of $\pi$ used in (5.1.2).

We now define $P_{a \otimes b}$ by the commutativity of

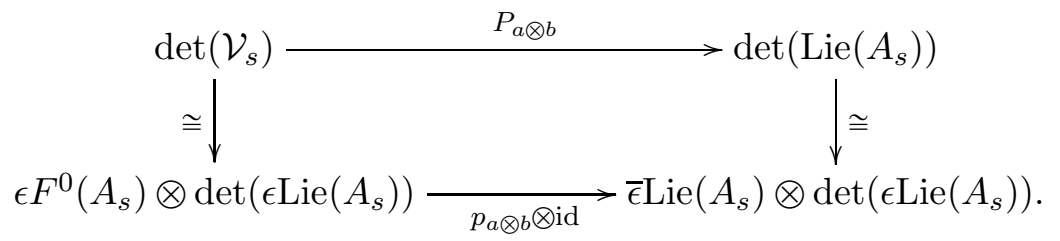

This defines the isomorphism

$$
\left(\bar{\epsilon} \operatorname{Lie}\left(A_{s}\right)\right)^{\otimes 2} \stackrel{P}{\rightarrow} \operatorname{Hom}\left(\operatorname{det}\left(\mathcal{V}_{s}\right), \operatorname{det}\left(\operatorname{Lie}\left(A_{s}\right)\right)\right)
$$

of [BHKRYa, Lemma 2.4.5].

Lemma 5.1.3. The isomorphism (5.1.9) defines an isometry

$$
\operatorname{det}\left(\mathcal{V}_{s}\right) \cong \mathcal{O}\left\langle 2 \pi D^{-1}\right\rangle_{s}^{\otimes 2} \otimes\left(\epsilon F^{0}\left(A_{s}\right)\right)^{\otimes 2} \otimes \operatorname{det}\left(\operatorname{Lie}\left(A_{s}\right)\right) .
$$

Proof. Fix an isomorphism $\bigwedge^{2 n} H_{1}\left(A_{s}(\mathbb{C}), \mathbb{Z}\right) \cong \mathbb{Z}$ and extend it to a $\mathbb{C}$ linear isomorphism

$$
\operatorname{vol}: \bigwedge^{2 n} H_{1}^{\mathrm{dR}}\left(A_{s}\right) \cong \mathbb{C}
$$

Under the de Rham comparison isomorphism $H_{1}\left(A_{s}(\mathbb{C}), \mathbb{C}\right) \cong H_{1}^{\mathrm{dR}}\left(A_{s}\right)$, the pairing (5.1.4) restricts to a perfect pairing

$$
\psi: H_{1}\left(A_{s}(\mathbb{C}), \mathbb{Z}\right) \times H_{1}\left(A_{s}(\mathbb{C}), \mathbb{Z}\right) \rightarrow 2 \pi i \mathbb{Z} .
$$

It follows that there is a unique element $\Psi=\alpha \wedge \beta \in \bigwedge^{2} H_{1}\left(A_{s}(\mathbb{C}), \mathbb{Z}\right)$ such that

$$
2 \pi i \cdot \psi(a, b)=\psi(\alpha, a) \psi(\beta, b)-\psi(\alpha, b) \psi(\beta, a)
$$

for all $a, b \in H_{1}\left(A_{s}(\mathbb{C}), \mathbb{Z}\right)$. The map

$$
\left(\bigwedge^{n-1} H_{1}\left(A_{s}(\mathbb{C}), \mathbb{Z}\right)\right) \otimes\left(\bigwedge^{n-1} H_{1}\left(A_{s}(\mathbb{C}), \mathbb{Z}\right)\right) \rightarrow \mathbb{Z}
$$

defined by $a \otimes b \mapsto \operatorname{vol}(\Psi \wedge a \wedge b)$ is a perfect pairing of $\mathbb{Z}$-modules.

We now metrize the line

$$
\operatorname{det}\left(\epsilon \operatorname{Lie}\left(A_{s}\right)\right) \subset \bigwedge^{n-1} \epsilon H_{1}^{\mathrm{dR}}\left(A_{s}\right)
$$


by $\|\mu\|^{2}=|\operatorname{vol}(\Psi \wedge \mu \wedge \bar{\mu})|$. With this definition, the vertical arrows in (5.1.8) are isometries.

Using (5.1.6) and (5.1.7), one sees that the map

$$
p_{a \otimes b} \in \operatorname{Hom}\left(F^{0}\left(A_{s}\right), \bar{\epsilon} \operatorname{Lie}\left(A_{s}\right)\right)
$$

satisfies $\left\|p_{a \otimes b}\right\|=2 \pi D \cdot\|a \otimes b\|$, and hence also $\left\|P_{a \otimes b}\right\|=2 \pi D \cdot\|a \otimes b\|$. This proves that the isomorphism $P$ defines an isometry

$$
\mathcal{O}\langle 2 \pi D\rangle_{s}^{\otimes 2} \otimes\left(\bar{\epsilon} \operatorname{Lie}\left(A_{s}\right)\right)^{\otimes 2} \cong \operatorname{Hom}\left(\operatorname{det}\left(\mathcal{V}_{s}\right), \operatorname{det}\left(\operatorname{Lie}\left(A_{s}\right)\right)\right) .
$$

The isomorphism (5.1.6) allows us to rewrite this as

$$
\operatorname{det}\left(\mathcal{V}_{s}\right) \cong \mathcal{O}\left\langle 2 \pi D^{-1}\right\rangle_{s}^{\otimes 2} \otimes\left(\epsilon F^{0}\left(A_{s}\right)\right)^{\otimes 2} \otimes \operatorname{det}\left(\operatorname{Lie}\left(A_{s}\right)\right) .
$$

The proof of BHKRYa, Proposition 2.4.2] gives an isomorphism

$$
\boldsymbol{\omega}_{s} \cong \operatorname{Hom}\left(\operatorname{Lie}\left(A_{0 s}\right), \epsilon F^{0}\left(A_{s}\right)\right) \subset \epsilon V_{\mathbb{C}}
$$

where

$$
V=\operatorname{Hom}_{\boldsymbol{k}}\left(H_{1}\left(A_{0 s}(\mathbb{C}), \mathbb{Q}\right), H_{1}\left(A_{s}(\mathbb{C}), \mathbb{Q}\right)\right) .
$$

As in [BHKRYa, $\S 2.1]$, there is a $\mathbb{Q}$-bilinear form $[\cdot, \cdot]: V \times V \rightarrow \mathbb{Q}$ induced by the polarizations on $A_{0 s}$ and $A_{s}$. If we extend this to a $\mathbb{C}$-bilinear form on

$$
V_{\mathbb{C}}=\operatorname{Hom}_{\boldsymbol{k} \otimes \mathbb{C}}\left(H_{1}^{\mathrm{dR}}\left(A_{0 s}\right), H_{1}^{\mathrm{dR}}\left(A_{s}\right)\right)
$$

then the metric on $\boldsymbol{\omega}_{s}$ is defined, as in [BHKRYa, $\S 7.2$ ], by

$$
\|x\|^{2}=\frac{|[x, \bar{x}]|}{4 \pi e^{\gamma}}
$$

for any $x \in \operatorname{Hom}\left(\operatorname{Lie}\left(A_{0 s}\right), \epsilon F^{0}\left(A_{s}\right)\right)$.

On the other hand, we have defined the Faltings metric on $\operatorname{Lie}\left(A_{0 s}\right)$, and defined a metric on $\epsilon F^{0}\left(A_{s}\right)$ by (5.1.5). The following lemma shows that (5.1.10) respects the metrics, up to scaling by a factor of $4 \pi e^{\gamma}$.

Lemma 5.1.4. The isomorphism (5.1.10) defines an isometry

$$
\mathcal{O}\left\langle 4 \pi e^{\gamma}\right\rangle_{s} \otimes \widehat{\boldsymbol{\omega}}_{s} \cong \operatorname{Hom}\left(\operatorname{Lie}\left(A_{0 s}\right), \epsilon F^{0}\left(A_{s}\right)\right) \text {. }
$$

Proof. The alternating form

$$
\psi_{0}: H_{1}^{\mathrm{dR}}\left(A_{0 s}\right) \times H_{1}^{\mathrm{dR}}\left(A_{0 s}\right) \rightarrow \mathbb{C}
$$

analogous to (5.1.4) restricts to a perfect pairing

$$
\psi_{0}: H_{1}\left(A_{0 s}(\mathbb{C}), \mathbb{Z}\right) \times H_{1}\left(A_{0 s}(\mathbb{C}), \mathbb{Z}\right) \rightarrow 2 \pi i \mathbb{Z},
$$

and hence the Faltings metric on $\operatorname{Lie}\left(A_{0 s}\right)=\epsilon H_{1}^{\mathrm{dR}}\left(A_{0 s}\right)$ is

$$
\|a\|^{2}=(2 \pi)^{-1}\left|\psi_{0}(a, \bar{a})\right| .
$$

From the definition of the bilinear form on $V$, one can show that

$$
[x, \bar{x}] \cdot \psi_{0}(a, \bar{a})=\psi(x a, \overline{x a})
$$


for all $x \in \epsilon V_{\mathbb{C}}$. Comparing with the metric on $\epsilon F^{0}\left(A_{s}\right)$ shows that

$$
4 \pi e^{\gamma} \cdot\|x\|^{2} \cdot\|a\|^{2}=(2 \pi)^{-1} \cdot|\psi(x a, \overline{x a})|=\|x a\|^{2},
$$

for all $x \in \boldsymbol{\omega}_{s}$ and $a \in \operatorname{Lie}\left(A_{0 s}\right)$, as claimed.

The two lemmas provide us with isometries

$$
\begin{aligned}
\operatorname{det}\left(\mathcal{V}_{s}\right) & \cong \mathcal{O}\left\langle 2 \pi D^{-1}\right\rangle_{s}^{\otimes 2} \otimes\left(\epsilon F^{0}\left(A_{s}\right)\right)^{\otimes 2} \otimes \operatorname{det}\left(\operatorname{Lie}\left(A_{s}\right)\right) \\
& \cong \mathcal{O}\left\langle 8 \pi^{2} e^{\gamma} D^{-1}\right\rangle_{s}^{\otimes 2} \otimes \widehat{\boldsymbol{\omega}}_{s}^{\otimes 2} \otimes \operatorname{Lie}\left(A_{0 s}\right)^{\otimes 2} \operatorname{det}\left(\operatorname{Lie}\left(A_{s}\right)\right)
\end{aligned}
$$

and the composition agrees with the isomorphism (5.1.3). This completes the proof of Proposition 5.1.2.

Recall the big CM cycle $\pi: \mathcal{Y}_{\text {big }} \rightarrow \mathcal{S}_{\text {Kra }}^{*}$ of Definition 4.2.6, All of the metrized line bundles on $\mathcal{S}_{\mathrm{Kra}}$ appearing in Proposition 5.1 .2 can be extended to the toroidal compactification $\mathcal{S}_{\text {Kra }}^{*}$ (with possible log-singularities along the boundary) so as to define classes in the codimension one arithmetic Chow group. However, we don't actually need this. Indeed, we can define a homomorphism

as the composition

$$
\left[-: \mathcal{Y}_{\text {big }}\right]: \widehat{\operatorname{Pic}}\left(\mathcal{S}_{\text {Kra }}\right) \rightarrow \mathbb{R}
$$

$$
\widehat{\operatorname{Pic}}\left(\mathcal{S}_{\mathrm{Kra}}\right) \stackrel{\pi^{*}}{\longrightarrow} \widehat{\operatorname{Pic}}\left(\mathcal{Y}_{\text {big }}\right) \cong \widehat{\mathrm{Ch}}^{1}\left(\mathcal{Y}_{\text {big }}\right) \stackrel{\widehat{\operatorname{deg}}}{\longrightarrow} \mathbb{R}
$$

As the big CM cycle does not meet the boundary of the toroidal compactification, the composition

$$
\widehat{\mathrm{Ch}}^{1}\left(\mathcal{S}_{\mathrm{Kra}}^{*}\right) \cong \widehat{\operatorname{Pic}}\left(\mathcal{S}_{\mathrm{Kra}}^{*}\right) \rightarrow \widehat{\operatorname{Pic}}\left(\mathcal{S}_{\mathrm{Kra}}\right) \stackrel{\left[-: \mathcal{Y}_{\text {big }}\right]}{\longrightarrow} \mathbb{R}
$$

agrees with the arithmetic degree along $\mathcal{Y}_{\text {big }}$ of Definition 4.2.6.

Remark 5.1.5. Directly from the definitions, and recalling Remark 2.2.7, the metrized line bundle $\mathcal{O}\langle c\rangle$ satisfies

$$
\left[\mathcal{O}\langle c\rangle: \mathcal{Y}_{\text {big }}\right]=\sum_{y \in \mathcal{Y}_{\text {big }}(\mathbb{C})}-\log \|1\|^{2}=-\log (c) \cdot \operatorname{deg}_{\mathbb{C}}\left(\mathcal{Y}_{\text {big }}\right) .
$$

5.2. The Faltings height. Recall from $\$ 4.2$ the moduli stack $\mathcal{C M}_{\Phi}$ of abelian varieties over $\mathcal{O}_{\Phi}$-schemes with complex multiplication by $\mathcal{O}_{E}$ and CM type $\Phi$.

Suppose $A \in \mathcal{C M}_{\Phi}(\mathbb{C})$. Choose a model of $A$ over a number field $L \subset \mathbb{C}$ large enough that the Néron model $\pi: \mathcal{A} \rightarrow \operatorname{Spec}\left(\mathcal{O}_{L}\right)$ has everywhere good reduction. Pick a nonzero rational section $s$ of the line bundle $\pi_{*} \Omega_{\mathcal{A} / \mathcal{O}_{L}}^{\operatorname{dim}(A)}$ on $\operatorname{Spec}\left(\mathcal{O}_{L}\right)$, and define

$$
h_{\infty}^{\text {Falt }}(A, s)=\frac{-1}{2[L: \mathbb{Q}]} \sum_{\sigma: L \rightarrow \mathbb{C}} \log \left|\int_{\mathcal{A}^{\sigma}(\mathbb{C})} s^{\sigma} \wedge \overline{s^{\sigma}}\right|,
$$

and

$$
h_{f}^{\text {Falt }}(A, s)=\frac{1}{[L: \mathbb{Q}]} \sum_{\mathfrak{p} \subset \mathcal{O}_{L}} \operatorname{ord}_{\mathfrak{p}}(s) \cdot \log \mathrm{N}(\mathfrak{p}) .
$$


By a result of Colmez Col93, the Faltings height

$$
h_{(E, \Phi)}^{\text {Falt }}=h_{f}^{\text {Falt }}(A, s)+h_{\infty}^{\text {Falt }}(A, s)
$$

depends only on the pair $(E, \Phi)$.

Proposition 5.2.1. The arithmetic degree of $\operatorname{Lie}(A)$ along $\mathcal{Y}_{\text {big }}$ satisfies

$$
\left[\operatorname{det}(\operatorname{Lie}(A)): \mathcal{Y}_{\text {big }}\right]=-2 \operatorname{deg}_{\mathbb{C}}\left(\mathcal{Y}_{\text {big }}\right) \cdot h_{(E, \Phi)}^{\text {Falt }} .
$$

Similarly, recalling the Faltings height $h_{\boldsymbol{k}}^{\text {Falt }}$ of 1.4.1),

$$
\left[\operatorname{Lie}\left(A_{0}\right): \mathcal{Y}_{\text {big }}\right]=-2 \operatorname{deg}_{\mathbb{C}}\left(\mathcal{Y}_{\text {big }}\right) \cdot h_{\boldsymbol{k}}^{\text {Falt }} .
$$

Proof. Suppose we are given a morphism $y: \operatorname{Spec}\left(\mathcal{O}_{L}\right) \rightarrow \mathcal{Y}_{\text {big }}$ for some finite extension $L / E_{\Phi}$. The restriction of $A$ to $\mathcal{O}_{L}$ has complex multiplication by $\mathcal{O}_{E}$ and CM type $\Phi$, and comparing the definition of the Faltings height with the definition of $\widehat{\operatorname{deg}}$ found in [How15, $§ 3.1]$, shows that the composition

$$
\widehat{\operatorname{Pic}}\left(\mathcal{S}_{\mathrm{Kra}}\right) \stackrel{\pi^{*}}{\longrightarrow} \widehat{\mathrm{Ch}}^{1}\left(\mathcal{Y}_{\mathrm{big}}\right) \stackrel{y^{*}}{\longrightarrow} \widehat{\mathrm{Ch}}^{1}\left(\operatorname{Spec}\left(\mathcal{O}_{L}\right)\right) \stackrel{\widehat{\mathrm{deg}}}{\longrightarrow} \mathbb{R}
$$

sends $\operatorname{Lie}(A)^{-1}$ to $[L: \mathbb{Q}] \cdot h_{(E, \Phi)}^{\text {Falt }}$.

We may choose $L$ in such a way that the $\mathcal{O}_{\boldsymbol{k}^{\text {-stack }}}$

$$
\mathcal{Y}_{\text {big }} \times_{\operatorname{Spec}\left(\mathcal{O}_{\Phi}\right)} \operatorname{Spec}\left(\mathcal{O}_{L}\right)
$$

admits a finite étale cover by a disjoint union $Y_{\text {big }}=\bigsqcup \operatorname{Spec}\left(\mathcal{O}_{L}\right)$ of, say, $m$ copies of $\operatorname{Spec}\left(\mathcal{O}_{L}\right)$, and then

$$
\frac{\left[\operatorname{Lie}(A): \mathcal{Y}_{\text {big }}\right]}{\operatorname{deg}_{\mathbb{C}}\left(\mathcal{Y}_{\text {big }}\right)}=\frac{\left[\operatorname{Lie}(A): Y_{\text {big }}\right]}{\operatorname{deg}_{\mathbb{C}}\left(Y_{\text {big }}\right)}=-\frac{m[L: \mathbb{Q}] \cdot h_{(E, \Phi)}^{\text {Falt }}}{m[L: \boldsymbol{k}]}=-2 \cdot h_{(E, \Phi)}^{\text {Falt }} .
$$

This proves the first equality, and the proof of the second is similar.

5.3. Gross's trick. The goal of $\$ 5.3$ is to compute the degree of the metrized line bundle $\operatorname{det}(\mathcal{V})$ along the big $\mathrm{CM}$ cycle. The impatient reader may skip directly to Proposition [5.3.6 for the answer. However, the strategy of the calculation is simple enough that we can explain it in a few sentences.

It is an observation of Gross Gro that the metrized line bundle $\operatorname{det}(\mathcal{V})$ behaves, for all practical purposes, like the trivial bundle $\mathcal{O}_{\mathcal{S}_{\text {Kra }}}$ endowed with the constant metric $\|1\|^{2}=\exp (-c)$ for a certain period $c$. This is made more precise in Theorem 5.3.1 and Corollary 5.3.2 below. A priori, the constant $c$ is something mysterious, but one can evaluate it by computing the degree of $\operatorname{det}(\mathcal{V})$ along any codimension $n-1$ cycle that one chooses. We choose a cycle along which the universal abelian scheme $A \rightarrow \mathcal{S}_{\mathrm{Kra}}$ degenerates to a product of CM elliptic curves. Using this, one can express the value of $c$ in terms of the Faltings height $h_{\boldsymbol{k}}^{\text {Falt }}$ appearing in (1.4.1). The degree of $\operatorname{det}(\mathcal{V})$ along $\mathcal{Y}_{\text {big }}$ is readily computed from this.

To carry out this procedure, the first step is to construct a cover of $\mathcal{S}_{\mathrm{Kra}}(\mathbb{C})$ over which the line bundle $\operatorname{det}(\mathcal{V})$ can be trivialized analytically. Fix a positive integer $m$, let $K(m) \subset K$ be the compact open subgroup of [BHKRYa, 
Remark 2.2.3], and consider the finite étale cover

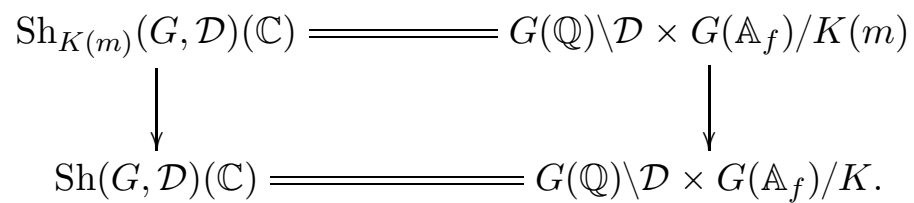

This cover has a moduli interpretation, exactly as with $\mathcal{S}_{\text {Kra }}$ itself, but with additional level $m$ structure. This allows us to construct a regular integral model $\mathcal{S}_{\mathrm{Kra}}(m)$ over $\mathcal{O}_{\boldsymbol{k}}[1 / m]$ of $\operatorname{Sh}_{K(m)}(G, \mathcal{D})$, along with a finite étale morphism

$$
\mathcal{S}_{\mathrm{Kra}}(m) \rightarrow \mathcal{S}_{\mathrm{Kra} / \mathcal{O}_{k}[1 / m]} .
$$

We use the notation $\operatorname{det}(\mathcal{V})$ for both the metrized line bundle on $\mathcal{S}_{\mathrm{Kra}}$, and for its pullback to $\mathcal{S}_{\mathrm{Kra}}(m)$.

The following results extends a theorem of Gross [Gro, Theorem 1] to integral models.

Theorem 5.3.1. Suppose $m \geqslant 3$, let $\mathbb{Z}^{\text {alg }} \subset \mathbb{C}$ be the subring of all algebraic integers, and fix a connected component

$$
\mathcal{C} \subset \mathcal{S}_{\mathrm{Kra}}(m)_{/ \mathbb{Z}^{\mathrm{alg}}[1 / m]} .
$$

The line bundle $\operatorname{det}(\mathcal{V})$ admits a nowhere vanishing section

$$
\eta \in H^{0}(\mathcal{C}, \operatorname{det}(\mathcal{V})) \text {. }
$$

Such a section is unique up to scaling by $\mathbb{Z}^{\text {alg }}[1 / m]^{\times}$, and its norm $\|\eta\|^{2}$ is constant on $\mathcal{C}(\mathbb{C})$.

Proof. For some $g \in G\left(\mathbb{A}_{f}\right)$ we have a complex uniformization

$$
\Gamma \backslash \mathcal{D} \stackrel{z \mapsto(z, g)}{\longrightarrow} \mathcal{C}(\mathbb{C}) \subset \mathrm{Sh}_{K(m)}(G, \mathcal{D})(\mathbb{C}),
$$

where $\Gamma=G(\mathbb{Q}) \cap g K(m) g^{-1}$, and under this uniformization the total space of the vector bundle $\operatorname{det}(\mathcal{V})$ is isomorphic to $\Gamma \backslash(\mathcal{D} \times \mathbb{C})$, where the action of $\Gamma$ on $\mathbb{C}$ is via the composition

$$
\Gamma \subset G(\mathbb{Q}) \rightarrow \mathrm{GL}(W) \stackrel{\text { det }}{\longrightarrow} \boldsymbol{k}^{\times} \subset \mathbb{C}^{\times} .
$$

The compact open subgroup $K(m)$ is constructed in such a way that there is a $\mathcal{O}_{\boldsymbol{k}}$-lattice $g \mathfrak{a} \subset W(\boldsymbol{k})$ stabilized by $\Gamma$, and such that $\Gamma$ acts trivially on $g \mathfrak{a} / m g \mathfrak{a}$. This implies that the above composition actually takes values in the subgroup

$$
\left\{\zeta \in \mathcal{O}_{\boldsymbol{k}}^{\times}: \zeta \equiv 1 \quad\left(\bmod m \mathcal{O}_{\boldsymbol{k}}\right)\right\},
$$

which is trivial by our assumption that $m \geqslant 3$. In other words, the vector bundle $\operatorname{det}(\mathcal{V})$ becomes (non-canonically) trivial after restriction to $\mathcal{X}(\mathbb{C})$. In fact, the argument of [Gro, Theorem 1] shows that one can find a trivializing section $\eta$ that is algebraic and defined over $\mathbb{Q}^{\text {alg }} \subset \mathbb{C}$, and that such a section is unique up to scaling by $\left(\mathbb{Q}^{\text {alg }}\right)^{\times}$and has constant norm $\|\eta\|^{2}$. 
All that remains to show is that $\eta$ may be chosen so that it extends to a nowhere vanishing section over $\mathbb{Z}^{\text {alg }}[1 / \mathrm{m}]$. The key is to recall from BHKRYa, $§ 2.3]$ that $\operatorname{Sh}(G, \mathcal{D})$ has a second integral model $\mathcal{S}_{\text {Pap }}$ over $\mathcal{O}_{\boldsymbol{k}}$, which is normal with geometrically normal fibers. It is related to the first by a surjective morphism $\mathcal{S}_{\text {Kra }} \rightarrow \mathcal{S}_{\text {Pap }}$, which restricts to an isomorphism over $\mathcal{O}_{\boldsymbol{k}}[1 / D]$. It has a moduli interpretation very similar to that of $\mathcal{S}_{\mathrm{Kra}}$, which allows us to do two things. First, there is a canonical descent of the vector bundle $\mathcal{V}$ to $\mathcal{S}_{\text {Pap }}$, defined again by $\mathcal{V}=H_{1}^{\mathrm{dR}}(A) / \bar{\epsilon} H_{1}^{\mathrm{dR}}(A)$, but where now $\left(A_{0}, A\right)$ is the universal pair over $\mathcal{S}_{\text {Pap }}$. Second, we can add level $K(m)$ structure to obtain a cartesian diagram

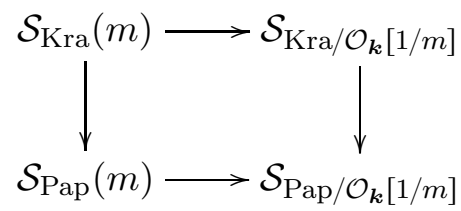

of $\mathcal{O}_{\boldsymbol{k}}[1 / m]$-stacks with étale horizontal arrows.

In particular, $\mathcal{S}_{\text {Pap }}(m)$ is normal with geometrically normal fibers, from which it follows that the above diagram extends to

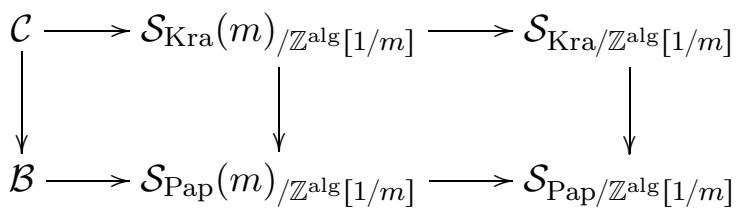

for some connected component $\mathcal{B} \subset \mathcal{S}_{\text {Pap }}(m)_{/ \mathbb{Z}^{\text {alg }}[1 / m]}$ with irreducible fibers.

Now fix a number field $L \subset \mathbb{C}$ containing $\boldsymbol{k}$ large enough that the section $\eta$ and the components $\mathcal{C}$ and $\mathcal{B}$ are defined over $\mathcal{O}_{L}[1 / m]$. Viewing $\eta$ as a rational section of the line bundle $\operatorname{det}(\mathcal{V})$ on $\mathcal{B}$, its divisor is a finite sum of vertical fibers of $\mathcal{B}$, and so there is a fractional $\mathcal{O}_{L}[1 / m]$-ideal $\mathfrak{b} \subset L$ such that

$$
\operatorname{div}(\eta)=\sum_{\mathfrak{q} \mid \mathfrak{b}} \operatorname{ord}_{\mathfrak{q}}(\mathfrak{b}) \cdot \mathcal{B}_{\mathfrak{q}}
$$

where $\mathcal{B}_{\mathfrak{q}}$ is the mod $\mathfrak{q}$ fiber of $\mathcal{Y}$. By enlarging $L$ we may assume that $\mathfrak{b}$ is principal, and hence $\eta$ can be rescaled by an element of $L^{\times}$to have trivial divisor on $\mathcal{B}$. But then $\eta$ also has trivial divisor on $\mathcal{C}$, as desired.

Corollary 5.3.2. Let $\mathcal{A} \subset \mathcal{S}_{\mathrm{Kra}}$ be a connected component. There is a constant $c=c_{\mathcal{A}} \in \mathbb{R}$ with the following property: for any finite extension $L / \boldsymbol{k}$ and any morphism $\operatorname{Spec}\left(\mathcal{O}_{L}\right) \rightarrow \mathcal{A}$, the image of $\operatorname{det}(\mathcal{V})$ under

$$
\widehat{\operatorname{Pic}}\left(\mathcal{S}_{\mathrm{Kra}}\right) \rightarrow \widehat{\operatorname{Pic}}(\mathcal{A}) \rightarrow \widehat{\operatorname{Pic}}\left(\operatorname{Spec}\left(\mathcal{O}_{L}\right)\right) \stackrel{\widehat{\mathrm{deg}}}{\rightarrow} \mathbb{R}
$$

is equal to $c \cdot[L: \boldsymbol{k}]$.

Proof. Fix an integer $m \geqslant 3$. The open and closed substack

$$
\mathcal{A}(m)=\mathcal{A} \times \mathcal{S}_{\mathrm{Kra}} \mathcal{S}_{\mathrm{Kra}}(m) .
$$


of $\mathcal{S}_{\mathrm{Kra}}(m)$, may be disconnected, so we fix one of its connected components $\mathcal{A}(m)^{\circ} \subset \mathcal{A}(m)$. This is an $\mathcal{O}_{\boldsymbol{k}}[1 / m]$-stack, which may become disconnected after base change to $\mathbb{Z}^{\text {alg }}[1 / m]$. Fix one connected component

$$
\mathcal{C} \subset \mathcal{A}(m)_{/ \mathbb{Z}^{\text {alg }}[1 / m]}^{\circ}
$$

and let $\eta \in H^{0}(\mathcal{C}, \operatorname{det}(\mathcal{V}))$ be a trivializing section as in Theorem 5.3.1

Choose a finite Galois extension $M / \boldsymbol{k}$ contained in $\mathbb{C}$, large enough that $\mathcal{C}$ and $\eta$ are defined over $\mathcal{O}_{M}[1 / m]$. For each $\sigma \in \operatorname{Gal}(M / \boldsymbol{k})$ we obtain a trivializing section

$$
\eta^{\sigma} \in H^{0}\left(\mathcal{C}^{\sigma}, \operatorname{det}(\mathcal{V})\right)
$$

which, by Theorem 5.3.1, has constant norm $\left\|\eta^{\sigma}\right\|$.

Let $\mathbb{R}(m)$ be the quotient of $\mathbb{R}$ by the $\mathbb{Q}$-span of $\{\log (p): p \mid m\}$, and define

$$
c(m)=\frac{-1}{[M: \boldsymbol{k}]} \sum_{\sigma \in \operatorname{Gal}(M / \boldsymbol{k})} \log \left\|\eta^{\sigma}\right\|^{2} \in \mathbb{R}(m) .
$$

This is independent of the choice of $M$, and also independent of $\eta$ by the uniqueness claim of Theorem 5.3.1. Moreover, for any number field $L / \boldsymbol{k}$ and any morphism

$$
\operatorname{Spec}\left(\mathcal{O}_{L}[1 / m]\right) \rightarrow \mathcal{A}(m)^{\circ}
$$

the image of $\operatorname{det}(\mathcal{V})$ under

$$
\widehat{\operatorname{Pic}}\left(\mathcal{A}(m)^{\circ}\right) \rightarrow \widehat{\operatorname{Pic}}\left(\operatorname{Spec}\left(\mathcal{O}_{L}[1 / m]\right)\right) \stackrel{\widehat{\operatorname{deg}}}{\longrightarrow} \mathbb{R}(m)
$$

is equal to $c(m) \cdot[L: \boldsymbol{k}]$.

Now suppose we are given some $\operatorname{Spec}\left(\mathcal{O}_{L}\right) \rightarrow \mathcal{A}$ as in the statement of the corollary. After possible enlarging $L$, this morphism admits a lift

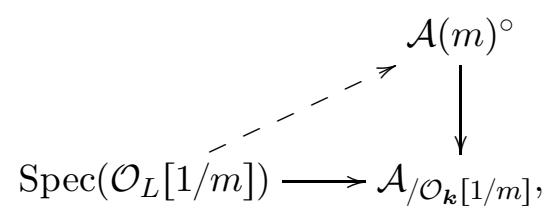

and from this it is easy to see that the image of $\operatorname{det}(\mathcal{V})$ under the composition of (5.3.1) with $\mathbb{R} \rightarrow \mathbb{R}(m)$ is equal to $c(m) \cdot[L: \boldsymbol{k}]$.

In particular, the image of $\operatorname{det}(\mathcal{V})$ under the composition of (5.3.1) with the diagonal embedding

$$
\mathbb{R} \hookrightarrow \prod_{m \geqslant 3} \mathbb{R}(m)
$$

is equal to the tuple of constants $c(m) \cdot[L: \mathbb{Q}]$. What this proves is that there is a unique $c \in \mathbb{R}$ whose image under the diagonal embedding is the tuple of constants $c(m)$, and that this is the $c$ we seek.

Proposition 5.3.3. The constant $c=c_{\mathcal{A}}$ of Corollary 5.3.2 is independent of $\mathcal{A}$, and is equal to

$$
c=(4-2 n) h_{\boldsymbol{k}}^{\text {Falt }}+\log \left(4 \pi^{2} D\right),
$$


where $h_{\boldsymbol{k}}^{\text {Falt }}$ is the Faltings height (1.4.1).

Proof. Recall that we have fixed a triple $\left(\mathfrak{a}_{0}, \mathfrak{a}, i_{E}\right)$ as in 4.2 . Fix a $g \in$ $G\left(\mathbb{A}_{f}\right)$ in such a way that the map

$$
\mathcal{D} \stackrel{z \mapsto(z, g)}{\longrightarrow} \operatorname{Sh}(G, \mathcal{D})(\mathbb{C})
$$

factors through $\mathcal{A}(\mathbb{C})$, and a decomposition of $\mathcal{O}_{\boldsymbol{k}}$-modules

$$
g \mathfrak{a}=\mathfrak{a}_{1} \oplus \cdots \oplus \mathfrak{a}_{n}
$$

in which each $\mathfrak{a}_{i}$ is projective of rank 1. Define elliptic curves over the complex numbers by

$$
A_{i}(\mathbb{C})=g \mathfrak{a}_{i} \backslash \mathfrak{a}_{i \mathbb{C}} / \bar{\epsilon} \mathfrak{a}_{i \mathbb{C}}
$$

for $0 \leqslant i<n$, and

$$
A_{n}(\mathbb{C})=g \mathfrak{a}_{n} \backslash \mathfrak{a}_{n} \mathbb{C} / \epsilon \mathfrak{a}_{n} \mathbb{C} .
$$

Endow the abelian variety $A=A_{1} \times \cdots \times A_{n}$ with the diagonal action of $\mathcal{O}_{\boldsymbol{k}}$, and the principal polarization induced by the perfect symplectic form on $g \mathfrak{a}$, as in the proof of [BHKRYa, Proposition 2.2.1]. The pair $\left(A_{0}, A\right)$ then corresponds to a point $(z, g) \in \mathcal{A}(\mathbb{C})$.

As each $A_{i}$ has complex multiplication by $\mathcal{O}_{\boldsymbol{k}}$, we may choose a number field $L$ containing $\boldsymbol{k}$ over which all of these elliptic curves are defined and have everywhere good reduction. If we denote again by $A_{0}, \ldots, A_{n}$ and $A$ the Néron models over $\operatorname{Spec}\left(\mathcal{O}_{L}\right)$, the pair $\left(A_{0}, A\right)$ determines a morphism

$$
\operatorname{Spec}\left(\mathcal{O}_{L}\right) \rightarrow \mathcal{A} \subset \mathcal{S}_{\mathrm{Kra}}
$$

The pullback of $\mathcal{V}$ to $\operatorname{Spec}\left(\mathcal{O}_{L}\right)$ is the rank $n$ vector bundle

$$
\left.\mathcal{V}\right|_{\operatorname{Spec}\left(\mathcal{O}_{L}\right)} \cong \mathcal{V}_{1} \oplus \cdots \oplus \mathcal{V}_{n}
$$

where $\mathcal{V}_{i}=H_{1}^{\mathrm{dR}}\left(A_{i}\right) / \bar{\epsilon} H_{1}^{\mathrm{dR}}\left(A_{i}\right)$. We endow $\mathcal{V}_{i}^{-1} \cong \epsilon H_{\mathrm{dR}}^{1}\left(A_{i}\right)$ with the metric (5.1.1), so that

$$
\left.\operatorname{det}(\mathcal{V})\right|_{\operatorname{Spec}\left(\mathcal{O}_{L}\right)} \cong \mathcal{V}_{1} \otimes \cdots \otimes \mathcal{V}_{n}
$$

is an isomorphism of metrized line bundles.

The following two lemmas relate the images of $\mathcal{V}_{1}, \ldots, \mathcal{V}_{n}$ under the arithmetic degree

$$
\widehat{\operatorname{Pic}}\left(\operatorname{Spec}\left(\mathcal{O}_{L}\right)\right) \stackrel{\widehat{\operatorname{deg}}}{\longrightarrow} \mathbb{R}
$$

to the Faltings height $h_{\boldsymbol{k}}^{\text {Falt }}$.

Lemma 5.3.4. For $1 \leqslant i<n$, the arithmetic degree (5.3.2) sends

$$
\mathcal{V}_{i} \mapsto-[L: \mathbb{Q}] \cdot h_{\boldsymbol{k}}^{\text {Falt }} .
$$

Proof. The action of $\mathcal{O}_{\boldsymbol{k}}$ on $\operatorname{Lie}\left(A_{i}\right)$ is through the inclusion $\mathcal{O}_{\boldsymbol{k}} \rightarrow \mathcal{O}_{L}$, and hence, as in BHKRYa, Remark 2.3.5], the quotient map

$$
H_{1}^{\mathrm{dR}}\left(A_{i}\right) \rightarrow \operatorname{Lie}\left(A_{i}\right)
$$


descends to an isomorphism of line bundles $\mathcal{V}_{i} \cong \operatorname{Lie}\left(A_{i}\right)$. If we endow $\operatorname{Lie}\left(A_{i}\right)^{-1}$ with the Faltings metric (5.1.1) then this isomorphism respects the metrics, and the claim follows as in the proof of Proposition 5.2.1.

Lemma 5.3.5. The arithmetic degree (5.3.2) sends

$$
\mathcal{V}_{n} \mapsto[L: \mathbb{Q}] \cdot\left(h_{\boldsymbol{k}}^{\text {Falt }}-\frac{1}{2} \log \left(4 \pi^{2} D\right)\right) .
$$

Proof. The action of $\mathcal{O}_{\boldsymbol{k}}$ on $\operatorname{Lie}\left(A_{i}\right)$ is through the complex conjugate of the inclusion $\mathcal{O}_{k} \rightarrow \mathcal{O}_{L}$, from which it follows that the Hodge short exact sequence takes the form

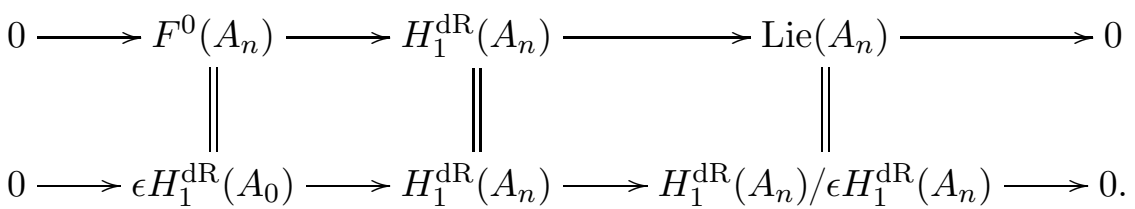

In particular, the endomorphism $\epsilon$ on $H_{1}^{\mathrm{dR}}\left(A_{n}\right)$ descends to an isomorphism $\mathcal{V}_{n} \cong F^{0}\left(A_{n}\right)$.

Let

$$
\psi_{n}: H_{1}^{\mathrm{dR}}\left(A_{n}\right) \otimes H_{1}^{\mathrm{dR}}\left(A_{n}\right) \rightarrow \mathcal{O}_{L}
$$

be the perfect pairing induced by the principal polarization on $A_{n}$, and define a second pairing $\Psi(x, y)=\psi_{n}(\epsilon x, y)$. It follows from the previous paragraph that this descends to a perfect pairing

$$
\Psi: \mathcal{V}_{n} \otimes \operatorname{Lie}\left(A_{n}\right) \cong \mathcal{O}_{L} .
$$

However, if we endow $\operatorname{Lie}\left(A_{n}\right)^{-1}$ with the Faltings metric (5.1.1), then this pairing is not a duality between metrized line bundles.

Instead, an argument as in the proof of Proposition 5.1.2 shows that

$$
\Psi: \mathcal{V}_{n} \otimes \operatorname{Lie}\left(A_{n}\right) \cong \mathcal{O}_{L}\left\langle\frac{1}{2 \pi \sqrt{D}}\right\rangle
$$

is an isomorphism of metrized line bundles. With this isomorphism in hand, the remainder of the proof is exactly as in the previous lemma.

The two lemmas show that the image of $\operatorname{det}(\mathcal{V})$ under (5.3.1) is

$$
\sum_{i=1}^{n} \widehat{\operatorname{deg}}\left(\mathcal{V}_{i}\right)=[L: \mathbb{Q}] \cdot\left((2-n) \cdot h_{k}^{\text {Falt }}-\frac{1}{2} \log \left(4 \pi^{2} D\right)\right)
$$

as claimed. This completes the proof of Proposition 5.3.3.

Proposition 5.3.6. The metrized line bundle $\operatorname{det}(\mathcal{V})$ satisfies

$$
\left[\operatorname{det}(\mathcal{V}): \mathcal{Y}_{\text {big }}\right]=\operatorname{deg}_{\mathbb{C}}\left(\mathcal{Y}_{\text {big }}\right) \cdot\left((4-2 n) h_{\boldsymbol{k}}^{\text {Falt }}+\log \left(4 \pi^{2} D\right)\right)
$$

Proof. As in the proof of Proposition [5.2.1, we may fix a finite extension $L / E_{\Phi}$ and a finite étale cover $Y_{\text {big }}=\bigsqcup \operatorname{Spec}\left(\mathcal{O}_{L}\right)$ of the $\mathcal{O}_{\boldsymbol{k}}$-stack

$$
\mathcal{Y}_{\text {big }} \times_{\operatorname{Spec}\left(\mathcal{O}_{\Phi}\right)} \operatorname{Spec}\left(\mathcal{O}_{L}\right)
$$


by, say, $m$ copies of $\operatorname{Spec}\left(\mathcal{O}_{L}\right)$. Corollary 5.3 .2 then implies

$$
\frac{\left[\operatorname{det}(\mathcal{V}): \mathcal{Y}_{\mathrm{big}}\right]}{\operatorname{deg}_{\mathbb{C}}\left(\mathcal{Y}_{\mathrm{big}}\right)}=\frac{\left[\operatorname{det}(\mathcal{V}): \mathrm{Y}_{\mathrm{big}}\right]}{\operatorname{deg}_{\mathbb{C}}\left(\mathrm{Y}_{\mathrm{big}}\right)}=\frac{\mathrm{cm} \cdot[L: \boldsymbol{k}]}{m \cdot[L: \boldsymbol{k}]}=c
$$

Appealing to the evaluation of the constant $c$ found in Proposition 5.3.3 completes the proof.

5.4. Theorems $\mathbf{C}$ and $\mathbf{D}$. We can now put everything together, and relate the arithmetic degree of $\widehat{\omega}$ along $\mathcal{Y}_{\text {big }}$ to the Faltings height $h_{(E, \Phi)}^{\text {Falt }}$.

Proposition 5.4.1. The metrized line bundle $\widehat{\boldsymbol{\omega}}$ satisfies

$$
\frac{\left[\widehat{\omega}: \mathcal{Y}_{\text {big }}\right]}{\operatorname{deg}_{\mathbb{C}}\left(\mathcal{Y}_{\text {big }}\right)}=h_{(E, \Phi)}^{\text {Falt }}+\frac{n-4}{2} \cdot \frac{\Lambda^{\prime}\left(0, \chi_{\boldsymbol{k}}\right)}{\Lambda\left(0, \chi_{\boldsymbol{k}}\right)}+\frac{n}{4} \log \left(16 \pi^{3} e^{\gamma}\right)
$$

Proof. Proposition 5.1 .2 shows that

$$
\begin{aligned}
2 \cdot\left[\mathcal{O}\left\langle 8 \pi^{2} e^{\gamma} D^{-1}\right\rangle \otimes \hat{\boldsymbol{\omega}}: \mathcal{Y}_{\text {big }}\right] & +\left[\operatorname{det}(\operatorname{Lie}(A)): \mathcal{Y}_{\text {big }}\right]+2 \cdot\left[\operatorname{Lie}\left(A_{0}\right): \mathcal{Y}_{\text {big }}\right] \\
& =\left[\mathcal{O}(\operatorname{Exc}): \mathcal{Y}_{\text {big }}\right]+\left[\operatorname{det}(\mathcal{V}): \mathcal{Y}_{\text {big }}\right] .
\end{aligned}
$$

Proposition 5.2.1 and Remark 5.1.5 imply that the left hand side is equal to

$$
2 \cdot\left[\widehat{\boldsymbol{\omega}}: \mathcal{Y}_{\text {big }}\right]-2 \operatorname{deg}_{\mathbb{C}}\left(\mathcal{Y}_{\text {big }}\right) \cdot\left(\log \left(8 \pi^{2} e^{\gamma} D^{-1}\right)+h_{(E, \Phi)}^{\text {Falt }}+2 \cdot h_{\boldsymbol{k}}^{\text {Falt }}\right),
$$

while Proposition 5.3.6 shows that the right hand side is equal to

$$
2 \operatorname{deg}_{\mathbb{C}}\left(\mathcal{Y}_{\text {big }}\right) \cdot\left((2-n) h_{\boldsymbol{k}}^{\text {Falt }}+\log (2 \pi D)\right) .
$$

Note that we have used here the equality

$$
\left[\mathcal{O}(\text { Exc }): \mathcal{Y}_{\text {big }}\right]=\left[\left(\text { Exc, 0) }: \mathcal{Y}_{\text {big }}\right]=\operatorname{deg}_{\mathbb{C}}\left(\mathcal{Y}_{\text {big }}\right) \cdot \log (D)\right.
$$

from the proof of Proposition 4.2.11,

Combining these formulas yields

$$
\frac{\left[\widehat{\boldsymbol{\omega}}: \mathcal{Y}_{\text {big }}\right]}{\operatorname{deg}_{\mathbb{C}}\left(\mathcal{Y}_{\text {big }}\right)}=h_{(E, \Phi)}^{\text {Falt }}+(4-n) h_{\boldsymbol{k}}^{\text {Falt }}+\log \left(16 \pi^{3} e^{\gamma}\right)
$$

and substituting the value (1.4.1) for $h_{\boldsymbol{k}}^{\text {Falt }}$ completes the proof.

It is clear from Proposition 5.4.1 that Theorems $\mathrm{C}$ and Theorem $\mathrm{D}$ are equivalent. As Theorem $\mathrm{C}$ is proved in [YY18, this completes the proof of Theorem D,

On the other hand, we proved Theorem $\mathrm{D}$ in 4.5 under the assumption that $n \geqslant 3$ and the discriminants of $\boldsymbol{k}$ and $F$ are odd and relatively prime, and so this gives a new proof of Theorem $\mathrm{C}$ under these hypotheses. 


\section{REFERENCES}

[AGHMP18] F. Andreatta, E.Z. Goren, B. Howard, and K. Madapusi Pera, Faltings heights of abelian varieties with complex multiplication, Ann. Math. (2) 187 (2018), no. 2, 391-531.

[Ami80] S.A. Amitsur, On the characteristic polynomial of a sum of matrices, Linear and Multilinear Algebra 8 (1979/80), no. 3, 177-182. MR 560557

[Asa76] T. Asai, On the Fourier coefficients of automorphic forms at various cusps and some applications to Rankin's convolution, J. Math. Soc. Japan 28 (1976), 48-61.

[BBGK07] J.H. Bruinier, J.I. Burgos Gil, and U. Kühn, Borcherds products and arithmetic intersection theory on Hilbert modular surfaces, Duke Math. J. 139 (2007), no. 1, 1-88.

[BF04] J.H. Bruinier and J. Funke, On two geometric theta lifts, Duke Math. J. 125 (2004), no. 1, 45-90.

[BGKK07] J.I. Burgos Gil, J. Kramer, and U. Kühn, Cohomological arithmetic Chow rings, J. Inst. Math. Jussieu 6 (2007), no. 1, 1-172. MR 2285241

[BHKRYa] J.H. Bruinier, B. Howard, S. Kudla, M. Rapoport, and T. Yang, Modularity of generating series of divisors on unitary Shimura varieties. This volume.

[BHY15] J.H. Bruinier, B. Howard, and T. Yang, Heights of Kudla-Rapoport divisors and derivatives of L-functions, Invent. Math. 201 (2015), no. 1, 1-95.

[BKY12] J.H. Bruinier, S.S. Kudla, and T. Yang, Special values of Green functions at big CM points, Int. Math. Res. Not. (2012), no. 9, 1917-1967.

[BY09] J.H. Bruinier and T. Yang, Faltings heights of CM cycles and derivatives of L-functions, Invent. Math. 177 (2009), no. 3, 631-681.

[Che14] G. Chenevier, The p-adic analytic space of pseudocharacters of a profinite group and pseudorepresentations over arbitrary rings, Automorphic forms and Galois representations. Vol. 1, London Math. Soc. Lecture Note Ser., vol. 414, Cambridge Univ. Press, Cambridge, 2014, pp. 221-285.

[Col93] P. Colmez, Périodes des variétés abéliennes à multiplication complexe, Ann. of Math. (2) 138 (1993), no. 3, 625-683.

[GS90] H. Gillet and C. Soulé, Arithmetic intersection theory, Inst. Hautes Études Sci. Publ. Math. (1990), no. 72, 93-174 (1991).

[Gro] B. Gross, On the periods of abelian integrals and a formula of Chowla and Selberg, Invent. Math. 45.

[GZ86] B. Gross and D. Zagier, Heegner points and derivatives of L-series, Inventiones Math. 84 (1986), pp. 225320.

[Har15] P. Hartwig, Kottwitz-Rapoport and p-rank strata in the reduction of Shimura varieties of PEL type, Ann. Inst. Fourier (Grenoble) 65 (2015), no. 3, 10311103.

[Hid04] H. Hida, p-adic automorphic forms on Shimura varieties, Springer Monographs in Mathematics, Springer Verlag, Berlin, Heidelberg, 2004.

[How12] B. Howard, Complex multiplication cycles and Kudla-Rapoport divisors, Ann. of Math. (2) 176 (2012), no. 2, 1097-1171.

[How15] Complex multiplication cycles and Kudla-Rapoport divisors II, Amer. J. Math. 137 (2015), no. 3, 639-698.

[How20] - On the averaged Colmez conjecture. Current Developments in Mathematics, 2018, International Press, (2020), pp. 125-178.

[HY12] B. Howard and T. Yang, Intersections of Hirzebruch-Zagier divisors and CM cycles, Lecture Notes in Mathematics, vol. 2041, Springer, Heidelberg, 2012.

[Jac62] R. Jacobowitz, Hermitian forms over local fields, Amer. J. Math., 84 (1962), 441-465. 
[Kud04] S. Kudla, Special cycles and derivatives of Eisenstein series, Heegner points and Rankin $L$-series, Math. Sci. Res. Inst. Publ., vol. 49, Cambridge Univ. Press, Cambridge, 2004, pp. 243-270.

[KR14] Special cycles on unitary Shimura varieties II: Global theory, J. Reine Angew. Math. 697 (2014), 91-157.

[Obu13] A. Obus, On Colmez's product formula for periods of CM-abelian varieties, Math. Ann. 356 (2013), no. 2, 401-418.

[Sch09] N.R. Scheithauer, The Weil representation of $\mathrm{SL}_{2}(\mathbb{Z})$ and some applications, Int. Math. Res. Not. IMRN (2009), no. 8, 1488-1545.

[Was82] L. Washington, Introduction to cyclotomic fields, Graduate Texts in Mathematics, vol. 83, Springer-Verlag, New York, 1982.

[Yan05] T. Yang, CM number fields and modular forms, Pure Appl. Math. Q. 1 (2005), no. 2, part 1, 305-340.

[YY18] T. Yang and H. Yin, CM fields of dihedral type and the Colmez conjecture, Manuscripta Math. 156 (2018), no. 1-2, 122.

[YZ18] X. Yuan and S.-W. Zhang, On the averaged Colmez conjecture, Ann. of Math. (2) 187 (2018), no. 2, 533638

[YZZ13] X. Yuan, S.-W. Zhang, and W. Zhang, The Gross-Zagier formula on Shimura curves, Annals of Mathematics Studies, vol. 184, Princeton University Press, Princeton, NJ, 2013.

Fachbereich Mathematik, Technische Universität Darmstadt, D-64289 DarmSTADT, GeRmany

E-mail address: bruinier@mathematik.tu-darmstadt.de

Department of Mathematics, Boston College, 140 Commonwealth Ave, ChestNUT HiLl, MA 02467, USA

E-mail address: howardbe@bc.edu

Department of Mathematics, University of Toronto, 40 St. George St., BA6290, Toronto, ON M5S 2E4, CANAdA

E-mail address: skudla@math.toronto.edu

Mathematisches Institut der Universität Bonn, Endenicher Allee 60, 53115 Bonn, Germany, and Department of Mathematics, University of Maryland, College PARK, MD 20742, USA

E-mail address: rapoport@math.uni-bonn.de

Department of Mathematics, University of Wisconsin Madison, Van Vleck Hall, Madison, WI 53706, USA

E-mail address: thyang@math.wisc.edu 